\author{
Universidade de São Paulo \\ Instituto de Física
}

\title{
O transistor válvula de spin de AlGaAs/GaAs e outros semicondutores: dirigido a novos dispositivos spintrônicos
}

\author{
Edgar Fernando Aliaga Ayllón
}

Orientador: Prof. Dr. Gennady Gusev

Dissertação apresentada ao Instituto de Física da Universidade de São Paulo para a obtenção do título de Mestre em Ciências.

\section{Banca examinadora:}

- Prof. Dr. Gennady Gusev (IFUSP)

- Prof. Dr. Félix Guillermo González Hernández (IFUSP)

- Prof. ${ }^{a}$ Dr. ${ }^{a}$ Ana Melva Champi Farfán (UFABC) 
FICHA CATALOGRÁFICA

Preparada pelo Serviço de Biblioteca e Informação do Instituto de Física da Universidade de São Paulo

Aliaga Ayllón, Edgar Fernando

O transistor válvula de spin de $\mathrm{AlGaAs} / \mathrm{GaAs}$ e outros semicondutores: dirigido a novos dispositivos spintrônicos - São Paulo, 2013.

Dissertação (Mestrado) - Universidade de São Paulo. Instituto de Física. Depto. Física dos Materiais e Mecânica.

Orientador: Prof. Dr. Gennary Gusev

Área de Concentração: Física da Matéria Condensada

Unitermos: 1. Gás de elétrons bidimensional 2. Poço parabólico; 3. Fator g de Landé; 4.Efeito Hall; 5. Válvula de spin. 
A Dios,

a mis padres Rosario y Edgar y a mis hermanos Juan Carlos y Miguel. 


\section{Agradecimentos}

Este trabalho foi possível graças ao grande número de pessoas que formaram parte da minha vida durante o processo. Primeiramente agradeço ao meus pais Rosario del Pilar Ayllón Ovalle e Edgar Aliaga Aliaga, pelo apoio constante e pela força que me deram em todo momento.

Ao Prof. Dr. Gennady Gusev por ter me orientado.

Aos professores Dr. Alexandre Levine e Dr. Felix Hernandez pelos conselhos e ajuda no trabalho de laboratorio.

Ao Prof. Dr. Nilo Mauricio Sotomayor Choque pela ajuda fornecida no estudo de calculos autoconsistentes e pela amizade na realização deste trabalho.

Ao Prof. Dr. Alain André Quivy, pelo crescimento das amostras.

Aos técnicos do laboratório, Sr. Francisco de Paula Oliveira (Paulinho) e Sr. Geraldo Chagas pelos conhecimentos fornecidos no trabalho com sistemas criogênicos e o apoio na construção dos equipamentos.

Ao meu amigo o Dr. Niko Churata Mamani (IFSC) por dispor-se sempre a executar os processos de litografia nas amostras, assim como pelo apoio e amizade.

A Antonio Augusto de Godoy Von Zuben (Totó) da Unicamp, pelos processos de evaporação de ouro nas amostras.

A Tatiana Lacerda Costa, secretaria do LNMS, por ter me ajudado sempre.

A Maria Izabel Cavalcante da Silva Albarracin e Éber de Patto Lima, bem como a todo o pessoal da comissão de Pós-Graduação. 
A mis hermanos Juan Carlos y Miguel, a mis queridos abuelos Carlos Ayllón y Ória Ovalle por el cariño y apoyo constante en la distancia; a mi ahijada Valentina que siempre me dará un motivo para sonreir.

Ao meu amigo Julio Bolaños pela amizade, colaboração e companhia nas tarefas experimentais; aos meus amigos de sala Abdur, Bruno Anghinoni, André dos Santos, Bruno Honda, Victor Manotas, Giovanni, Jorge Leon, Zahra, Lilian, Thiago, Gerson pela convivência sempre agradável.

Aos meus amigos do IFUSP, Lindber, Martín, Denis, Miguel, Carlos, Heyner, Hector, René, Jośe, Victor, Laura, Noemi, Tania, Pablo Badilla e Dennis Bredes pelo companherismo, amizade e hospitalidade em todos estes anos.

A Natalia per avermi dato la forza, il sostegno, la comprensione, la gioia e l'amore nei tempi che ho bisogno di più in questi ultimi mesi di lavoro.

Ao Laboratório de Novos Materiais Semicondutores (LNMS) do Instituto de Física da USP, por ter fornecido as facilidades necessárias para a conclusão deste trabalho.

E por fim, agradecer a CNPq pelo apoio financieiro, sem o qual não teria condições de realizar este trabalho. 


\section{Resumo}

Neste trabalho, apresentamos estudos de magnetotransporte em um sistema quase tridimensional de elétrons produzido em amostras contendo poços quânticos parabólicos (PQW, Parabolic Quantum Well) formados em heteroestruturas de AlGaAs crescidos sobre substratos de GaAs pela técnica de epitaxia por feixe molecular (MBE).

Na primeira parte do nosso trabalho realizamos medidas de magnetoresistência, efeito Hall e efeito Shubnikov-de Haas em PQWs com larguras de $1000 \AA$ a fim de investigar as propriedades eletrônicas tais como a concentração e a mobilidade dos elétrons nas amostras. Através de cálculos autoconsistentes determinou-se os perfis de potencial, os níveis de energia e as concentrações de cada uma das sub-banda ocupadas no poço. Uma análise através da transformada de Fourier também permitiu determinar as concentrações eletrônicas nas sub-bandas.

Em uma segunda parte estudou-se a influência da aplicação de potenciais externos através de uma porta metálica com barreira em uma amostra contendo um PQW de largura $3000 \AA$ na presença de campos magnéticos perpendicular e paralelo à superficie da amostra. Encontrou-se que para uma tensão de porta de $V_{g}=-0,55 \mathrm{~V}$ forma-se uma barreira de potencial ainda sem ter depleção de cargas no poço.

Apresenta-se a idealização do dispositivo transistor válvula de spin, a partir do fato que aplicando uma tensão de porta é possível deslocar espacialmente os elétrons e mudar a sua orientação de spin. 


\section{Abstract}

Results from magnetic transport studies made on quasi-three-dimensional electron systems are presented in this work. AlGaAs heterostructures grown on GaAs subtrates through molecular beam epitaxy (MBE) enable the existence of this type of systems by means of parabolic quantum wells (PQW) formation.

This work was developed in two main parts. First, we studied magnetoresistence phenomena, such as Hall effect and Shubnikov-de Haas, on $1000 \AA$ width PQWs. This permits to know the electronic concentration and mobility values of this type of samples, among other electrical properties. Then, self-consistent calculations gave an outline of the size and shape of the potentials, and gave the values for the energy levels and the electronic concentration on each occupied sub-band of the quantum well. Through Fourier transform analysis was also possible to obtain and confirm the electronic concentrations of the occupied sub-bands.

In the second part of the work, we studied the effects of applying an external potential through a barrier gate to a $3000 \AA$ width PQW sample in the presence of magnetic fields parallel and perpendicular to the sample surface. For a $V g=-0,55 \mathrm{~V}$ gate voltage, it was found that a potential barrier was formed even without charge depletion in the well.

An idealization for the spin valve transistor device, based on the fact that applying a gate potential spatially dislocates the electrons and changes their spin orientation, is presented. 


\section{Sumário}

Introdução $\quad$ xix

1 Sistema Bidimensional de Elétrons (2DES) em Campos Magnéticos 1

1.1 2DES em Heteroestruturas de AlGaAs/GaAs . . . . . . . . . . . . . . . . 1

1.1.1 Sistema bidimensional de elétrons em campo magnético zero . . . . 3

1.1.2 Estrutura eletrônica em campo magnético perpendicular . . . . . . 4

1.1.3 Estrutura eletrônica em campo magnético paralelo . . . . . . . . . 7

1.2 Transporte Semiclássico . . . . . . . . . . . . . . . . . . . . . . . . 11

1.2.1 Equação de Bolztmann . . . . . . . . . . . . . . . . . . . . . . 11

1.2 .2 Modelo de Drude . . . . . . . . . . . . . . . . . . . . . 12

1.2.3 Escalas de Comprimento Relevantes . . . . . . . . . . . . . . . . 13

1.2.4 Efeito Hall Clássico . . . . . . . . . . . . . . . . . . . . . . . . . . . 17

1.3 Transporte Quântico . . . . . . . . . . . . . . . . . . . . 20

1.3.1 O efeito Hall quântico inteiro . . . . . . . . . . . . . . . . 20

1.3.2 O efeito Shubnikov-de Haas . . . . . . . . . . . . . . . . . . . . . 24

1.3.3 Transporte de estados de borda . . . . . . . . . . . . . . . 27

1.4 Fator g de Landé em $A l_{x} G a_{1-x} A s \ldots$. . . . . . . . . . . . . . . 32

1.5 Estados eletrônicos dos poços quânticos parabólicos na presença de campo magnético . . . . . . . . . . . . . . . . . . . 35

1.6 Idealização do transistor válvula de spin . . . . . . . . . . . . . . . . . 41

2 Estrutura Eletrônica dos Poços Quânticos Parabólicos de AlGaAs 43

2.1 Poços quânticos parabólicos . . . . . . . . . . . . . . . . . . . 43

2.2 Cálculos Autoconsistentes . . . . . . . . . . . . . . . . . . 47

2.3 Cálculos autoconsistentes para PQW . . . . . . . . . . . . . 50

2.4 Cálculos dos poços empregados . . . . . . . . . . . . . . . . 55

3 Crescimento, Processamento e Caracterização das Amostras 59

3.1 Crescimento por MBE . . . . . . . . . . . . . . . . . . . 59

3.2 Arquitetura dos poços quânticos parabólicos . . . . . . . . . . . . . . . 61

3.3 Processamento das amostras . . . . . . . . . . . . . . . . . . . . 63 
3.3.1 Gravação da barra Hall . . . . . . . . . . . . . . . . . . . . . . 63

3.3.2 Difusão dos contatos ôhmicos . . . . . . . . . . . . . . . . . 65

3.3.3 Evaporação da porta metálica com barreira . . . . . . . . . . . . 65

3.4 Caracterização por meio de transporte . . . . . . . . . . . . . . . . 68

3.4.1 Equipamentos utilizados . . . . . . . . . . . . . . . . 68

3.4 .2 Técnicas de caracterização de amostras . . . . . . . . . . . . . . 70

4 Análise e Discussão de Resultados $\quad 73$

4.1 Medidas em poços quânticos parabólicos . . . . . . . . . . . . . . . 74

4.1.1 Resultados para a amostra A2578 . . . . . . . . . . . . . 74

4.1.2 Resultados para a amostra A2580 . . . . . . . . . . . . . 78

4.2 Medidas em poços quânticos parabólicos com porta com barreira . . . . . . 81

$\begin{array}{lll}5 & \text { Conclusões } & 97\end{array}$

$\begin{array}{ll}\text { Referências } & 99\end{array}$ 


\section{Lista de Figuras}

1.1 Camadas de AlGaAs - GaAs, com uma região de AlGaAs dopada (tipo $n)$; considerando só a diferença nos gaps desses materiais a interface deve ter essa forma quando a energia de Fermi é a mesma ao longo da estrutura.

1.2 Representação da relação de dispersão para um gas de elétrons bidimensional. 4

1.3 (a) Orbitas ciclotrônicas no espaço $k$. (b) Evolução da densidade de estados com o incremento do campo magnético. . . . . . . . . . . . . . . . . . . . . 6

1.4 (a) Estrutura de banda de superfície $(i=0,1,2)$ no limite elétrico $\left(k_{D} l_{B} \gg 1\right)$. A ressonância ciclotrônica é somente possível para centros de coordenadas positivos $\left(z_{0} / l \gtrsim+1\right)$. (b) Estrutura de banda de superfície $(i=0,1)$ no limite magnético $\left(k_{D} l_{B} \ll 1\right)$. A figura no extremo superior direito representa o contorno de Fermi para $E_{F}=1 / 4 \hbar \omega_{c}$. A ressonância ciclotrônica é possível para a maior parte dos centros de coordenadas. . . . . . . . . . .

1.5 Trajetórias dos elétrons para regimes de transporte difusivo $(l<W, L)$, quase balístico $(W<l<L)$ e balístico $(l>W, L) \ldots \ldots \ldots \ldots$

1.6 Semicondutor em forma retangular ilustrando o efeito Hall clássico. . . . . 18

1.7 Diagrama mostrando a região de estados localizados e estados estendidos nos níveis de Landau. Também mostra-se o desdobramento Zeeman de spin. 22

1.8 (a) Barra Hall e a configurão para medidas de $\rho_{x x}$ e $\rho_{x y}$. (b) Medidas típicas de magnetorresistência longitudinal e resistência de Hall. . . . . . . . . . . 23

1.9 Oscilações Shubnikov-de Haas da amostra A2580 de GaAs/AlGaAs contendo um poço quântico parabólico. . . . . . . . . . . . . . . . 25

1.10 Trajetórias clássicas dos elétrons movendo-se ao longo da borda de uma amostra na presença de campo magnético. . . . . . . . . . . . . 27

1.11 Mostra-se a dependência da posição dos espectros de energia de um 2DEG na presença de um campo magnético, em unidades da energia do cíclotron $E_{c}=\hbar \omega_{c}$. A linha pontilhada horizontal representa a energia de Fermi; neste caso existem dois níveis de Landau abaixo da $E_{F}$ no Bulk. As linhas pontilhadas verticalmente representam as posições das bordas da amostra.

1.12 Esquema de uma barra Hall num campo magnético perpendicular com dois estados de borda $(\nu=2)$ em cada lado da amostra. . . . . . . . . . . . . 30 
1.13 Modelo de 5 níveis para as bandas de condução e valência do GaAs perto do ponto $\Gamma$ na zona de Brillouin. Estão indicados as simetrias dos níveis em relação ao ponto $\Gamma_{i}$, os gaps de energia $E_{i}$ e as energias de spin-órbita $\Delta_{i}$. $P_{0}$ e $P_{1}$ são os elementos da matriz momento. O zero de energia é escolhido no ponto $\Gamma_{c}^{6} \ldots \ldots \ldots \ldots \ldots \ldots \ldots$

1.14 Comparação entre os valores do fator $g$ de Landé em função da concentração de $A l$, calculados pelo modelo de 5 bandas da teoria k.p (preto) e pela fórmula de Weisbuch e Hermann (vermelho), para a liga de $A l_{x} G a_{(1-x)} A s$.

1.15 Níveis de energia de um poço quântico parabólico em função do campo magnético paralelo. A linha tracejada mostra a posição do nível de Fermi em campo magnético zero. . . . . . . . . . . . . . . . . . 36

1.16 Mostra-se o espectro de energia para um poço quântico parabólico em função do campo magnético perpendicular. (a) Em campos magnéticos intensos. (b) Em campos magnéticos baixos. A posição do nível de Fermi em campo magnético zero é mostrada pela linha tracejada. As energias de ciclotron e sub-bandas são indicadas. . . . . . . . . . . . . . . . . . 38

1.17 Mostra-se a dependência do fator $g$ com a composição de $A l$ na liga $A l_{x} G a_{1-x} A s$ para $x \leq 0,3 \ldots \ldots \ldots \ldots \ldots \ldots$

1.18 (a) Representação esquemática da orientação de spin dos elétrons em um poço quântico parabólico na presença de campo magnetico paralelo intenso. (b) Variação do fator $g$ de Landé ao longo do eixo z. . . . . . . . . . . . . . 40

1.19 Mostra-se o esboço do transistor válvula de spin. Um fluxo de corrente $I$ atravessa um poço quântico parabólico submetido a um campo magnético $B$ paralelo à superficie da amostra. São mostradas também as órbitas ciclotrônicas dos elétrons com raios muito menor do que a largura do poço (gás 3D de elétrons). De acordo com a localização da densidade eletrônica, o fator $g$ será positivo ou negativo, de modo que os elétrons adquirem spin "up" (aneis roxos) ou "down" (aneis vermelhos), para que a energia de Zeeman seja mínima. A tensão de porta manipula a localização eletrônica.

2.1 (a) Poço quântico estreito, o sistema possui caráter bidimensional. (b) Poço quântico largo, sistema separado em duas regiões cada uma com caráter bidimensional. (c) Potencial parabólico. (d) PQW com uma camada de elétrons homogénea quase tridimensional. . . . . . . . . . . . . . . . . . . . 44

2.2 Esquema representativo que ilustra o perfil de potencial e os parâmetros relacionados com um poço parabólico. Mostra-se o caso de um poço com

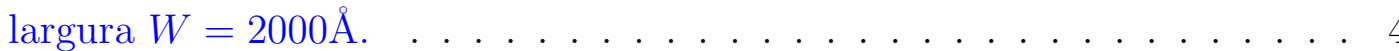


2.3 Resultados de cálculos autoconsistentes para um PQW de 500Å. Mostra-se o perfil da banda de condução $V_{p}(z)$ em preto, o potencial eletrostático de repulsão coulombiana $V_{c}(z)$ em azul, e o potencial de troca e correlação $V_{x c}(z)$ em verde. O potencial autoconsistente total é mostrado em vermelho. A linha tracejada em preto é a distribuição da densidade eletrônica. . . . . .

2.4 Distribuição da densidade de carga para cada uma das sub-bandas e a densidade de carga total para um poço de $W=2000 \AA$ e $n_{s}=2,0 \times 10^{11}$ $\mathrm{cm}^{-2}$.

2.5 Perfil de potencial total e a densidade de carga para um poço de $W=2000 \AA$ e $n_{s}=2,0 \times 10^{11} \mathrm{~cm}^{-2}$. No gráfico do potencial não foi considerada a barreira. 51

2.6 Perfil do potencial autoconsistente convergido e a densidade de carga total para um poço de $W=2000 \AA$ e $n_{s}=3,9 \times 10^{11} \mathrm{~cm}^{-2} \ldots \ldots \ldots \ldots$. . . . . 52

2.7 Distribuição da densidade de carga para cada uma das sub-bandas e a densidade de carga total para um poço de $W=1000 \AA$ e $n_{s}=4,2 \times 10^{11}$ $\mathrm{cm}^{-2}$.

2.8 Potencial autoconsistente total e a densidade de carga total para um poço de $W=1000 \AA$ e $n_{s}=4,2 \times 10^{11} \mathrm{~cm}^{-2} \ldots \ldots \ldots \ldots \ldots$. . . . . 54

2.9 Potencial autoconsistente total e a densidade de carga total para um poço de $W=1000 \AA$ e $n_{s}=2,1 \times 10^{11} \mathrm{~cm}^{-2} \ldots \ldots \ldots \ldots$

2.10 Distribuição da densidade de carga para cada uma das sub-bandas e a densidade de carga total para um poço de $W=1000 \AA$ e $n_{s}=2,1 \times 10^{11}$ $\mathrm{cm}^{-2}$.

2.11 Potencial autoconsistente total convergido e a distribuição de densidade de carga total para um poço de $W=1000 \AA$ e $n_{s}=3,2 \times 10^{11} \mathrm{~cm}^{-2}$. . . . . .

2.12 Distribuição da densidade de carga para cada uma das sub-bandas e a densidade de carga total para um poço de $W=1000 \AA$ e $n_{s}=3,2 \times 10^{11}$ $\mathrm{cm}^{-2} \ldots \ldots \ldots \ldots \ldots \ldots \ldots \ldots$

3.1 Representação esquemática do crescimento de amostras através da técnica de Epitaxia por feixe molecular $(\mathrm{MBE}) \ldots$. . . . . . . . . . . . . . .

3.2 Esquema genérico de um sistema MBE onde são crescidas as heteroestruturas semicondutoras. . . . . . . . . . . . . . . . . . . .

3.3 Representação esquemática da sequencia das camadas semicondutoras que compõem as amostras de poços parabólicos, assim como a concentração de alumínio na liga. . . . . . . . . . . . . . . . . . . .

3.4 Desenho da barra Hall litografada nas amostras. Mostra-se a região de interesse com largura de $200 \mu \mathrm{m}$ e comprimento de $500 \mu \mathrm{m}$. 
3.5 Equipamento para fazer a difusão dos contatos ôhmicos na amostra com barra Hall. (a) Módulo eletrônico que controla a temperatura da câmara da amostra. (b) Câmara da amostra, contem um sistema de aquecimento e é mantida numa atmosfera de $N_{2}$ gasoso. (c) Microscópio para colocar os contatos de In . . . . . . . . . . . . . . . . . . . . . . . . . . . . 65

3.6 Mostra-se o esquema da deposição de camadas de PMMA sobre a barra Hall com a barreira feita por feixe de elétrons, e a camada de ouro (porta). 66

3.7 (a) Câmara de evaporação do ouro e titânio. (b) Região da amostra abaixo do acetato que fica exposta ao fluxo de ouro/titânio. . . . . . . . . . . . . . 67

3.8 Esquema de uma amostra com porta ou gate pronta para as medidas de transporte. . . . . . . . . . . . . . . . . . . 68

3.9 Esquema interno básico do criostato com bobina supercondutora para medidas de transporte no LNMS. . . . . . . . . . . . . . . . . . . . . . . 69

3.10 Desenho da barra Hall, onde a corrente elétrica é aplicada através dos contatos 1 (fonte) e 4 (dreno). A região de interesse é acessada por os contatos, através dos quais efetuamos as medidas de magnetorresistência e efeito Hall. . . . . . . . . . . . . . . . . . . . . . . . . . . . . . . 71

4.1 Oscilações da magnetoresistência $\left(R_{x x}\right)$ e resistência de Hall $\left(R_{x y}\right)$ para a amostra A2578, a uma temperatura de 1,5K. . . . . . . . . . . . . . 74

4.2 (a) Oscilações Shubnikov-de Haas da amostra A2578. (b) Oscilações Shubnikovde Haas em função do inverso do campo magnético, elas são periodicas. 75

4.3 Espectro de frequências da transformada de Fourier das oscilações Shubnikovde Haas da amostra A2578. . . . . . . . . . . . . . . . . . . . . . . 76

4.4 Oscilações da magnetoresistência $\left(R_{x x}\right)$ e resistência de Hall $\left(R_{x y}\right)$ para a amostra A2580, a uma temperatura de 1,5K . . . . . . . . . . 78

4.5 (a) Oscilações Shubnikov-de Haas da amostra A2580. (b) Oscilações Shubnikovde Haas em função do inverso do campo magnético, elas são periodicas. 79

4.6 Espectro de frequências da transformada de Fourier das oscilações Shubnikovde Haas da amostra A2580. . . . . . . . . . . . . . . . . . . . . . . . . . . 80

4.7 Esquema do modelo de estados de borda para uma amostra com barreira. Nesse caso, é aplicada uma tensão de porta negativa $\left(V_{g}<0\right)$ que gera uma reflexão dos estados de borda mais internos na barreira. Nas regiões não perturbadas temos fator de preenchimento $\nu=2$, e na região da barreira

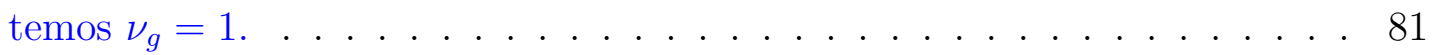

4.8 Magnetoresistência na amostra A2519 para uma voltagem de porta $V_{g}=$ $-0,2 \mathrm{~V}$ em campo magnético perpendicular. . . . . . . . . . . . 82

4.9 Magnetoresistência na amostra A2519 para uma voltagem de porta $V_{g}=$ $-0,4 V$ em campo magnético perpendicular. . . . . . . . . . . . . . 83 
4.10 Magnetoresistência da amostra A2519 em função da voltagem de porta para diferentes valores de campo magnético. O gráfico está em escala logarítmica. 84

4.11 Gráficos tridimensionais da magnetoresistência para a amostra A2519 em função da voltagem de porta $V_{g}$ e do campo magnético $B$ paralelo. (a) Mostra-se o gráfico da magnetoresistência em escala linear. (b) Mostra-se o gráfico da magnetoresistência em escala logarítmica. . . . . . . . . . . . . 84

4.12 (a) Magnetoresistência $R_{x x}$ e curva ajustada $R_{x x}^{A j u s t e}$ para $B=0,0 \mathrm{~T}$. (b) Magnetoresistência $R_{x x}$ e curva ajustada $R_{x x}^{A j u s t e}$ para $B=8,5 \mathrm{~T} \ldots \ldots$. . 85

4.13 Variação de $R_{0}$ com o campo magnético. Mostra-se também o ajuste linear para a região $B>1,5 \mathrm{~T}$. . . . . . . . . . . . . . . . . 87

4.14 Variação de $a$ com o campo magnético. Mostra-se também o ajuste exponencial para a região $B>1,5 \mathrm{~T}$. . . . . . . . . . . . . . . 87

4.15 Variação de $b$ com o campo magnético. Mostra-se também o ajuste linear para a região $B>1,5 \mathrm{~T} \ldots \ldots \ldots \ldots$. . . . . . . . . . 88

4.16 Magnetoresistência em função da tensão de porta e do campo magnético para $B>1,5 T$ obtida a partir da Eq.(4.8). (a) Gráfico em escala linear. (b) Gráfico em escala logarítmica. . . . . . . . . . . . . . . . . . . . . 89

4.17 Esquema do canal de corrente da barra Hall onde são mostradas as dimensões da barreira (em amarelho). Tal largura da barreira gera um fator de forma $f=1,75 \times 10^{-3} \ldots \ldots \ldots$. . . . . . . . . . . 90

4.18 Mostra-se a resistência quadrado na região da barreira em função da tensão de porta para diferentes valores de campo magnético. O gráfico está em escala logarítmica. . . . . . . . . . . . . . . . . . . . 9 90

4.19 Mostra-se o gráfico tridimensional em escala logarítmica da $R_{\square}$ na região da barreira em função do campo (B) e da tensão de porta $\left(V_{g}\right)$. . . . . . . . 91

4.20 Dependência de $\mu^{-1}$ com a concentração de Alumínio (x) a partir dos dados de G. C. Gardner et. al. Mostra-se também o ajuste linear. . . . . . . . . . 92

4.21 Mostra-se o gráfico da dependência da mobilidade $\mu$ com a densidade de carga $n_{s}$ para $\left|V_{g}\right|>0,55 \ldots \ldots \ldots \ldots \ldots$. . . . . . . . . . . 95 
xvi LISTA DE FIGURAS 


\section{Lista de Tabelas}

1.1 Algumas escalas de comprimento relevantes. Notar que $1 \mu \mathrm{m}=10^{-6} \mathrm{~m}=$ $10^{-4} \mathrm{~cm} ; 1 \mathrm{~nm}=10^{-9} \mathrm{~m}=10 \AA \ldots \ldots \ldots \ldots \ldots$

2.1 Energias e densidades de carga para um poço parabólico com parâmetros: $W=2000 \AA, n_{s}=2,0 \times 10^{11} \mathrm{~cm}^{-2}, \Delta_{1}=155 \mathrm{meV}$ e $\Delta_{2}=75 \mathrm{meV}$. Neste poço três sub-bandas estão ocupadas. . . . . . . . . . . . . . . . . 50

2.2 Energias e densidades de carga para um poço parabólico com parâmetros: $W=2000 \AA, n_{s}=3,9 \times 10^{11} \mathrm{~cm}^{-2}, \Delta_{1}=155 \mathrm{meV}$ e $\Delta_{2}=75 \mathrm{meV}$. Este poço tem 5 sub-bandas ocupadas. . . . . . . . . . . . . . . 52

2.3 Energias e densidades de carga para um poço parabólico com parâmetros: $W=1000 \AA, n_{s}=4,2 \times 10^{11} \mathrm{~cm}^{-2}, \Delta_{1}=162,5 \mathrm{meV}$ e $\Delta_{2}=97,7 \mathrm{meV}$. Neste poço três sub-bandas estão ocupadas. . . . . . . . . . . . . . . 53

2.4 Energias e densidades de carga para um poço parabólico com parâmetros: $W=1000 \AA, n_{s}=2,1 \times 10^{11} \mathrm{~cm}^{-2}, \Delta_{1}=162,5 \mathrm{meV}$ e $\Delta_{2}=97,7 \mathrm{meV}$. Neste poço duas sub-bandas estão ocupadas, mas a segunda se encontra quase vazia. . . . . . . . . . . . . . . . . . . . . 56 56

2.5 Energias e densidades de carga para um poço parabólico com parâmetros: $W=1000 \AA, n_{s}=3,2 \times 10^{11} \mathrm{~cm}^{-2}, \Delta_{1}=162,5 \mathrm{meV}$ e $\Delta_{2}=97,7 \mathrm{meV}$. Neste poço duas sub-bandas estão ocupadas. . . . . . . . . . . . . . . . 58

4.1 Amostras estudadas no presente trabalho. . . . . . . . . . . . . . . 73

4.2 Mostra-se os valores dos parâmetros $R_{0}, a$ e $b$ para cada campo magnético

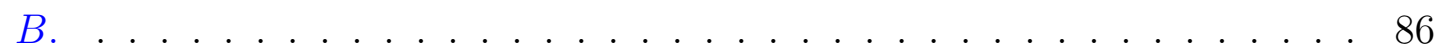

4.3 Mostra-se os valores de mobilidade $\mu$ obtidos a partir da Eq.(4.9) para valores de concentração de Alumínio variando desde 0,05 (centro do poço) até 0,30 (valor aproximado de $x$ na barreira do nosso poço parabólico). . 93

4.4 Mobilidades experimentais a campo magnético zero para diferentes valores de tensão de porta. Elas são cálculadas a partir dos valores de resistência da Fig.(4.10) e assumindo que $n_{s}$ é constante. . . . . . . . . . . . . . . . . 93 
4.5 Mostra-se as mobilidades $\mu$ e as densidades de carga $n_{s}$ para alguns valores de tensão de porta. Na região $\left|V_{g}\right|<0,55, n_{s}$ é constante; mas na região $\left|V_{g}\right|>0,55, n_{s}$ diminui. . . . . . . . . . . . . . . . 94 


\section{Introdução}

Os progressos na fabricação de estruturas semicondutoras de elevada pureza atingiram um nível tal que as camadas monoatômicas de diferentes materiais podem ser crescidas uma sobre a outra, de modo que a estrutura cristalina pode ser mantida em uma extensão muito grande. Assim, com a síntese artificial dessas estruturas, foi possível produzir sistemas eletrônicos que confinam o movimento dos portadores em certas direções. Em tais estruturas, os elétrons experimentam efeitos quânticos que modificam fortemente o seu comportamento. Em particular, a investigação de um gás de elétrons confinado em um plano (o chamado gás bidimensional de elétrons, 2DEG) tem levado a descobertas fundamentais na área. O efeito Hall quântico inteiro, descoberto por K. von Klitzing em 1980 [1] e, posteriormente, o efeito Hall quântico fracionário [2], representam os fenômenos quânticos mais notáveis que surgiram a partir de varias décadas de intenso estudo dos 2DEG.

Entende-se por heteroestrutura semicondutora um material artificialmente produzido, o qual é formado pela superposição de camadas alternadas de semicondutores distintos. O ponto de partida para a idealização das heteroestruturas foi o trabalho de L. Esaki e R. Tsu em 1970 [3], onde propuseram o crescimento de super-redes para a observação do tunelamento ressonante. Assim, deu-se início de uma longa linha de pesquisa e desenvolvimento que resultou nos modernos dispositivos semicondutores usados atualmente na eletrônica e na optoeletrônica. As primeiras heteroestruturas foram produzidas por Blakeslee e Aliotta [4] (1970) usando a técnica de CVD (Chemical Vapor Deposition) crescendo estruturas de GaAlAs/GaAs $s_{1-x} P_{x}$. Posteriormente, A. Y. Cho [5] (1971) e W. P. Dumke [6] (1972) cresceram estruturas de $G a A s / A l_{1-x} G a_{x} A s$ usando as técnicas de MBE (Molecular Beam Epitaxy) e LPE (Liquid Phase Epitaxy), respectivamente. Estas novas técnicas de crescimento permitiram a produção de amostras monocristalinas contendo um número preciso de camadas atômicas, com interfaces abruptas entre materiais semicondutores distintos, perfis de dopagem sofisticados, alta pureza, e baixo número de defeitos estruturais. Atualmente, heteroestruturas crescidas em diversas arquiteturas formam a base de uma série de aparelhos, amplamente utilizados em nosso cotidiano, que utilizam a tecnologia dos semicondutores. 
Com o aprimoramento das técnicas de crescimento, começou-se a pensar no desenvolvimento de heteroestruturas onde o gap do material da região ativa pudesse ser controlado criando estruturas com perfis de potencial sofisticados. Assim, foi possível o crescimento dos poços quânticos parabólicos (PQW, Parabolic Quantum Well) empregados neste trabalho. Este tipo de estrutura foi proposta inicialmente por Miller [7] em 1984, mas Sundaram et al. [8] e Shayegan et al. [9] foram os primeiros a crescer este tipo de potencial. $\mathrm{O}$ atual progresso alcançado nas técnicas de crescimento por epitaxia de feixe molecular, tem possibilitado a construção experimental dos poços quânticos parabólicos remotamente dopados para estudos de transporte quântico, devido ao fato do poço quântico parabólico ser um sistema promissor para a formação de um gás de elétrons quase-tridimensional, uniforme e com alta mobilidade. Atualmente, a produção deste tipo de sistema tem evoluido significativamente, e já é possível encontrar um grande número de trabalhos dedicados à investigação das propriedades do gás de elétrons quase-tridimensional.

A idéia básica por trás destas estruturas é a criação de um perfil de banda de condução na direção do crescimento das heteroestruturas baseadas nas ligas $G a A s / A l_{x} G a_{1-x} A s$, através da variação da fração molar de $A l$, de modo a simular o potencial parabólico de uma camada com distribuição uniforme da carga positiva. Esta é uma pseudo-carga, a qual tem uma densidade proporcional à curvatura do poço. A injeção remota de portadores de carga, provenientes das barreiras dopadas, produz a blindagem da pseudo-carga no interior do poço formando uma camada eletrônica uniforme, e com alta mobilidade, ao longo da camada correspondente no poço.

Motivados pela possibilidade do controle e a manipulação do spin do elétron, com os quais se possa desenvolver uma nova tecnologia complementar à eletrônica do silício e demais semicondutores, neste trabalho desenvolvemos estudos com heteroestruturas semicondutoras de poços quânticos parabólicos (PQW). Devido ao acoplamento spin órbita, o fator $g$ de Landé em materiais bulk difere do valor $g_{0}$ de um elétron livre $\left(g_{0}=2,0023\right)$. Uma vez que o fator $g$ de Landé muda com a concentração de alumínio, e também com a direção de crescimento $z$ em um poço parabólico, temos que o fator $g$ pode assumir valores tanto positivos como negativos ao longo da largura de um poço parabólico. Assim, estas estruturas representam fortes candidatos para o desenvolvimento de dispositivos destinados à spintrônica.

A tese é apresentada da seguinte maneira:

O primeiro capítulo descreve as propriedades dos gáses bidimensionais de elétrons submetidos à aplicação de campos magnéticos uniformes nas configurações perpendicular e paralelo. A seguir apresentamos uma revisão de alguns fenômenos de transporte semi- 
clássico e transporte quântico que nos auxiliarão na análise do comportamento dos gáses confinados nos poços quânticos parabólicos. Também apresenta-se uma outra descrição do efeito Hall quântico baseada no modelo de estados de borda. É introduzido o fator $g$ de Landé. Mostra-se os estados eletrônicos dos poços quânticos parabólicos na presença de campo magnético. Finalmente é introduzido a idealização do transistor válvula de spin.

O segundo capítulo apresenta o modelo teórico utilizado para descrever a estrutura eletrônica dos poços quânticos parabólicos. A seguir, descreve-se um programa que resolve de forma autoconsistente as equações de Schrodinger e Poisson, usado para o cálculo dos níveis de energia, mostrando-se também os resultados com ele obtidos. O programa foi desenvolvido por um ex-membro do nosso grupo de pesquisa, o Dr. Nilo Mauricio Sotomayor Choque atualmente professor na Universidade Federal de Tocantins.

O terceiro capítulo apresenta primeiramente a técnica de crescimento MBE. A seguir descreve-se a arquitetura dos poços quânticos parabólicos, os processamentos nas amostras como a gravação da barra Hall e a evaporação da porta de ouro, e finalmente os procedimentos básicos utilizados para a realização das medidas de transporte na presença de campos magnéticos.

O quarto capítulo apresenta a análise e discução das medidas feitas nas nossas amostras de poços quânticos parabólicos. Em uma primeira parte, estudou-se os resultados das medidas em amostras de poços parabólicos sem porta metálica, submetidos à aplicação de campo magnético perpendicular. Na segunda parte, mostra-se os resultados das medidas em amostras contendo uma porta metálica com barreira submetidos a campos magnéticos perpendicular e paralelo.

No quinto capítulo apresentamos as concluções do nosso estudo. 
xxii INTRODUÇÃO 


\section{Capítulo 1}

\section{Sistema Bidimensional de Elétrons (2DES) em Campos Magnéticos}

\subsection{DES em Heteroestruturas de AlGaAs/GaAs}

Um sistema bidimensional de elétrons (Two-dimensional electron system, 2DES) ou gás bidimensional de elétrons (Two-dimensional electron gas, 2DEG) tem como caracteristica o fato que o movimento destes portadores é livre em duas direções do espaço (por exemplo no plano $x y$ ) sendo confinados na terceira direção espacial (por exemplo na direção do eixo z). Portanto, num sistema bidimensional, os elétrons estarão confinados por algum potencial $V(z)$. Sua primeira constatação foi em um MOSFET (Metal Oxid Semiconductor Field Effect Transistor) em 1960 por Kahng e Atalla [10]. Isto permitiu a descoberta de novos efeitos físicos, tais como as oscilações magnéticas da condutância [11] ou o efeito Hall quântico [1, 2], que levou dois prêmios Nobel em 1985 e 1998.

A maneira mais comum para obter um sistema bidimensional é numa estrutura formada por uma interface entre um material semicondutor e um material isolante, ou entre dois materiais semicondutores. Um exemplo é mostrado na Fig.(1.1). O potencial formado nestes tipos de estruturas permite o confinamiento de elétrons na direção $z$ perpendicular ao plano da interface. Assim, a movimentação dos elétrons na direção $z$ é quantizada em estados discretos de energia, enquanto no plano $x-y$ a movimentação é livre. Os níveis de energia confinados na direção $z$ são chamados de sub-bandas.

Uma das estruturas mais básicas para confinar elétrons na banda de condução é uma interface formada entre dois materiais semicondutores com diferentes gaps de energia. Por exemplo, o perfil das bandas de energia de uma estrutura AlGaAs/GaAs é mostrado na Fig.(1.1). Para poder confinar elétrons na banda de condução, a estrutura básica pode ser uma camada de $A l G a A s$ com dopagem tipo $n$ seguida de outra camada de AlGaAs sem dopagem e finalmente a camada de GaAs para formar a interface. 
Assim, a banda de condução tem elétrons livres fornecidos pela camada dopada. Uma vez que o potencial de banda de condução na região do GaAs é menor que na região do $A l G a A s$, os elétrons na região dopada de $A l G a A s$ migram para a banda de condução do GaAs. Aparece então, uma carga positiva na região das impurezas doadoras, devido à ionização delas, a qual atrai os elétrons para a interface, criando uma curvatura na banda de condução. A transferencia de elétrons desde a camada dopada de AlGaAs até a camada de GaAs continua até que a descontinuidade do potencial permita ter o mesmo nível de Fermi na camada de $A l G a A s$ e na camada de GaAs, ver Figura (1.1). Os elétrons na banda de condução abaixo do nível de Fermi permanecem confinados em estados quantizados.
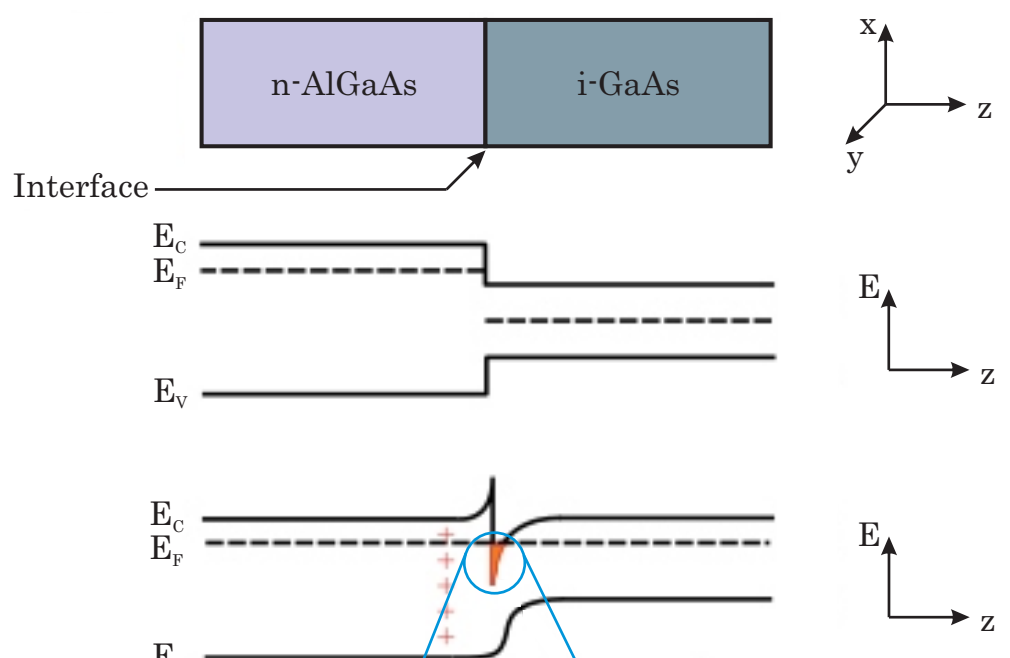

Figura 1.1: Camadas de AlGaAs - GaAs, com uma região de AlGaAs dopada (tipo n); considerando só a diferença nos gaps desses materiais a interface deve ter essa forma quando a energia de Fermi é a mesma ao longo da estrutura. 


\subsubsection{Sistema bidimensional de elétrons em campo magnético zero}

Nestes sistemas de semicondutores bidimensionais, a equação de Schrodinger que descreve o movimento dos elétrons na direção do crescimento é dada por:

$$
\left[-\frac{\hbar^{2}}{2 m^{*}} \nabla^{2}+V(z)\right] \Psi(\boldsymbol{r})=E \Psi(\boldsymbol{r})
$$

Uma vez que o potencial de confinamento é independente das coordenadas $x-y$, a equação de Schrodinger é separável em z. Assim é possível obter as soluções para o potencial $V(z)$ independentemente de $x$ e $y$, e o potencial $V(z)$ confina os elétrons em estados quantizados. Vamos usar a seguinte função de onda tentativa:

$$
\Psi(\rho, z)_{k, n}=\frac{1}{\sqrt{S}} \phi_{n}(z) e^{i k_{x} x} e^{i k_{y} y}=\phi(z) \psi(\rho)
$$

onde $\rho$ é o vetor $(x, y), k_{x}$ e $k_{y}$ são as componentes do vetor de onda e $1 / \sqrt{S}$ é uma constante de normalização para estas funções sendo $S$ a área da amostra na direção perpendicular ao crescimento, isto é $S=L_{x} L_{y}$. Substituindo a Eq.(1.2) na Eq.(1.1) obtemos a relação de dispersão da energia no espaço $k$ :

$$
E_{n, k_{x}, k_{y}}=E_{n}+\frac{\hbar^{2} k_{x}^{2}}{2 m^{*}}+\frac{\hbar^{2} k_{y}^{2}}{2 m^{*}}
$$

onde o número inteiro $n=0,1,2, \ldots$ indica os auto-valores de energia na direção $z$. Estes níveis de energias quantizados $E_{n}$ definem os mínimos de cada sub-banda. Em baixa temperatura e baixa densidade de elétrons, todos os elétrons ocupam a primeira sub-banda, assim, um sistema bidimensional de elétrons é formado.

A densidade de estados por unidade de área de cada uma das sub-bandas ocupadas é constante (ver Fig.(1.2)) e pode ser expressa por:

$$
\rho\left(E_{n}\right)=\frac{m^{*}}{\pi \hbar^{2}}
$$

A densidade de estados total do sistema quase-bidimensional de elétrons $D(E)$, é dada pela soma das densidades parciais de cada uma das sub-bandas ocupadas, o qual é expresso por:

$$
D(E)=\sum_{n} \rho\left(E_{n}\right)
$$


Quando o sistema tem só uma sub-banda ocupada, o sistema é considerado um sistema perfeitamente bidimensional e quando o sistema tem duas ou mais sub-bandas ocupadas, é chamado quase-bidimensional.
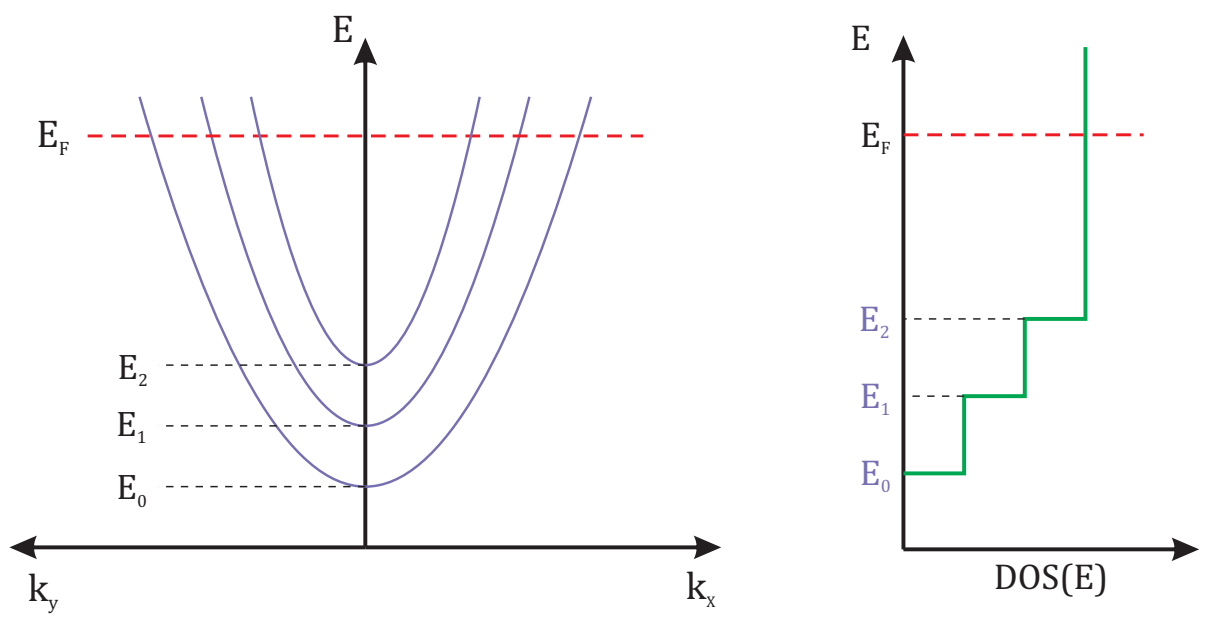

Figura 1.2: Representação da relação de dispersão para um gas de elétrons bidimensional.

\subsubsection{Estrutura eletrônica em campo magnético perpendicular}

Quando é aplicado um campo magnético $B_{z}$, perpendicular ao plano de crescimento (plano da interface), a movimentação dos elétrons no plano $x-y$ é livre e no eixo $z$ é quantizado em órbitas com níveis de energia discretos chamados de níveis de Landau. Esta quantização do sistema bidimensional tem uma influência grande nas suas propriedades devido à densidade de estados ter uma mudança significativa.

Para uma estrutura bidimensional com perfil de potencial arbitrário na banda de condução $V(z)$, se considera um campo magnético aplicado na direção $z$. A equação de Schrodinger fica assim:

$$
\left[\frac{1}{2 m^{*}}(-i \hbar \nabla+e \mathbf{A})^{2}+V(z)\right] \Psi(x, y, z)=E \Psi(x, y, z)
$$

Considerando o gauge para o potencial vetor: $\mathbf{A}=(-y B, 0,0)$ de tal forma que $\mathbf{B}=$ $\nabla \times \mathbf{A}=B \hat{k}$, então substituindo $\mathbf{A}$ e $\Psi(x, y, z)=\psi(x, y) \phi(z)$ obtemos:

$\frac{1}{2 m^{*} \psi(x, y)}\left[-\hbar^{2}\left(\frac{\partial^{2}}{\partial x^{2}}+\frac{\partial^{2}}{\partial y^{2}}\right)+e^{2} B^{2} y^{2}\right] \psi(x, y)+\frac{-\hbar^{2}}{2 m^{*} \phi(z)} \frac{\partial^{2} \phi(z)}{\partial z^{2}}+\frac{1}{\phi(z)} V(z) \phi(z)=E$ 
Então a equação (1.7) pode ser separada em duas equações independentes:

$$
\frac{1}{2 m^{*}}\left[-\hbar^{2}\left(\frac{\partial^{2}}{\partial x^{2}}+\frac{\partial^{2}}{\partial y^{2}}\right)+e^{2} B^{2} y^{2}\right] \psi(x, y)=E^{\prime} \psi(x, y)
$$

e

$$
\left[\frac{-\hbar^{2}}{2 m^{*}} \frac{\partial^{2}}{\partial z^{2}}+V(z)\right] \phi(z)=E_{z} \phi(z)
$$

Onde: $E=E^{\prime}+E_{z}$

A equação (1.8) pode ser expressa como:

$$
\frac{1}{2 m^{*}}\left[-\hbar^{2} \frac{\partial^{2}}{\partial y^{2}}+\left(\frac{\hbar}{i} \frac{\partial}{\partial x}-e B y\right)^{2}\right] \psi(x, y)=E^{\prime} \psi(x, y)
$$

Desta ultima equação, como o Hamiltoniano não depende de $x$, então a função de onda $\psi(x, y)$ pode ser escrita como o produto de uma onda plana em $x$ e uma função não conhecida $u(y)$ :

$$
\psi(x, y)=e^{i k_{x} x} u(y)
$$

Então substituindo temos:

$$
\frac{1}{2 m^{*}}\left[-\hbar^{2} \frac{\partial^{2} u(y)}{\partial y^{2}}+\left(\hbar k_{x}-e B y\right)^{2} u(y)\right]=E^{\prime} u(y)
$$

a qual fica:

$$
\left[-\frac{\hbar^{2}}{2 m^{*}} \frac{\partial^{2}}{\partial y^{2}}+\frac{1}{2} m^{*} \omega_{c}^{2}\left(y-y_{0}\right)^{2}\right] u(y)=E^{\prime} u(y)
$$

Onde $\omega_{c}=e B / m^{*}$ é a frequencia de cíclotron, $y_{0}=l_{0}^{2} k_{x}$ é o centro da órbita ciclotrônica, com $l_{0}=\sqrt{\hbar / e B}$ que é o comprimento magnético.

Esta ultima equação é a de um oscilador harmônico centrado em $y_{0}$, então a função de onda da equação (1.11) fica assim: $\psi(x, y)=e^{i k_{x} x} \Phi_{n}\left(y-y_{0}\right)$ e a energia será $E^{\prime}=$ $(n+1 / 2) \hbar \omega_{c}$.

Então a equação de onda total para o gás bidimensional de elétrons é:

$$
\Psi(x, y, z)=\phi(z) \Phi_{n}\left(y-y_{0}\right) e^{i k_{x} x}
$$


Na discussão acima, nós desprezamos o efeito do campo magnético sobre as variáveis de spin dos elétrons. Na verdade, um termo extra deve ser adicionado ao Hamiltoniano da equação (1.6): $H=s g \mu_{B} B[12]$

A quantização orbital dos níveis de energia pode ser expressa como:

$$
E_{n, k_{z}}=E_{k_{z}}+\left(n+\frac{1}{2}\right) \hbar \omega_{c}+s g \mu_{B} B
$$

onde $n=0,1,2, \ldots, s= \pm 1 / 2$ é o número quântico de spin, $g$ é o fator de Landé, e $\mu_{B}$ é o magneton de Bohr. A Figura (1.3) mostra as orbitas ciclotrônicas para os elétrons no espaço $k$, a correspondente densidade de estados e a evolução da DOS na presença de campo magnético crescente.

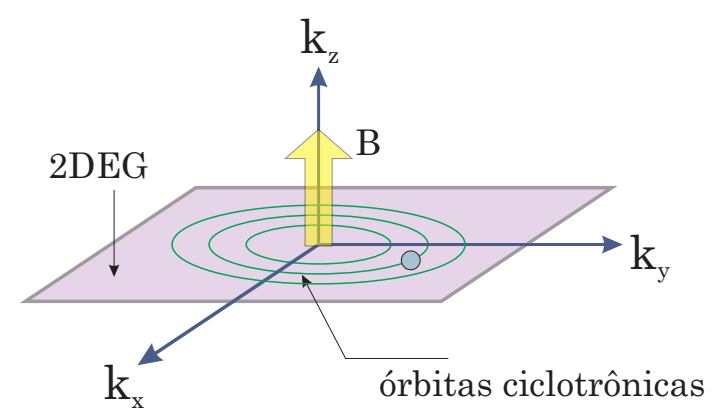

(a)

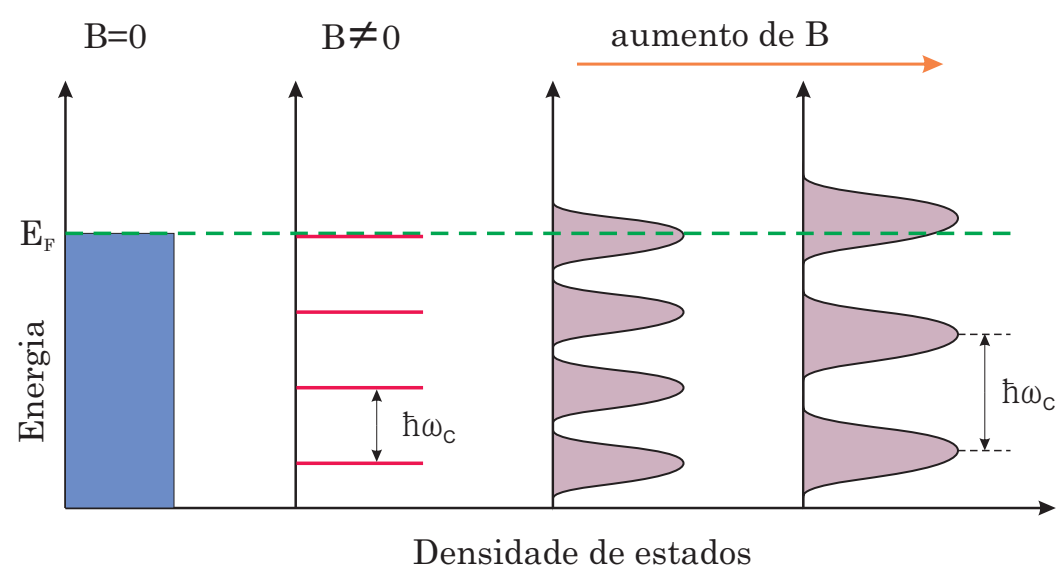

(b)

Figura 1.3: (a) Orbitas ciclotrônicas no espaço $k$. (b) Evolução da densidade de estados com o incremento do campo magnético. 


\subsubsection{Estrutura eletrônica em campo magnético paralelo}

Considerando uma heterojunção de $G a A s / A l_{x} G a_{1-x} A s$, a qual confina um gás bidimensional de elétrons em um plano perpendicular ao eixo $z$. Se o campo magnético $B=B_{x}$ está orientado ao longo da direção $x$, o Hamiltoniano de um elétron confinado no plano $x-y$ por um potencial $V_{c}(z)$ é expresso por:

$$
H=\frac{1}{2 m^{*}}(\vec{p}-e \vec{A})^{2}+V_{c}(z)
$$

onde $m^{*}$ é a massa efetiva eletrônica, $\vec{p}$ é o operador momento, $e$ é a carga do elétron, e $\vec{A}$ é o potencial vetor, o qual pode ser expresso na forma:

$$
\vec{A}=\left(0,-B_{x} z, 0\right)
$$

em forma explícita o Hamiltoniano do sistema pode-se escrever como:

$$
H=\frac{1}{2 m^{*}}\left(p_{y}-e B_{x} z\right)^{2}+\frac{p_{x}^{2}}{2 m^{*}}+\frac{p_{z}^{2}}{2 m^{*}}+V_{c}(z)
$$

O espectro de energia é formado por sub-bandas e os autovalores de energia $E_{n}\left(k_{x}, k_{y}\right)$, $n=0,1, \ldots$ são funções do vetor de onda quase-contínuo $k_{x}$ e $k_{y}$. As superfícies de Fermi bidimensionais são definidas separadamente para cada sub-banda.

O campo magnético paralelo não influencia o movimento dos elétrons na direção $y$, no entanto, na direção $x$ os elétrons são acelerados ou desacelerados pela ação combinada dos campos cruzados $B_{x}$ e $E_{z}=-d V_{c}(z) / d z$, dependendo da forma do potencial de confinamento. Para o caso particular de um poço parabólico $V_{c}(z)=\frac{1}{2} m^{*} \omega_{0}^{2} z^{2}$, submetido a ação de um campo magnético paralelo, os autovalores de energia podem ser achados em forma analítica [13],

$$
E_{n}\left(p_{x}, p_{y}\right)=\left(n+\frac{1}{2}\right) \hbar \tilde{\omega}+\frac{1}{2 m^{*}} p_{x}^{2}+\frac{1}{2 m_{y}^{*}} p_{y}^{2}
$$

ou equivalentemente [14]:

$$
E_{n}\left(k_{x}, k_{y}\right)=\left(n+\frac{1}{2}\right) \hbar \tilde{\omega}+\frac{\hbar^{2}}{2 m^{*}}\left(k_{x}^{2}+\frac{\omega_{0}^{2}}{\tilde{\omega}^{2}} k_{y}^{2}\right)
$$

onde $n=0,1, \ldots, \tilde{\omega}=\left(\omega_{x}^{2}+\omega_{0}^{2}\right)^{\frac{1}{2}}, \omega_{x}=\frac{|e| B_{x}}{m^{*}}$, e $m_{y}^{*}=\frac{m^{*} \tilde{\omega}^{2}}{\omega_{0}^{2}}$, é a nova massa efetiva induzida para a componente $y$ do movimento eletrônico, a qual cresce com $B_{x}$. 
O campo magnético paralelo influencia o movimento dos elétrons nas direções $x$ e $z$, mas não muda o movimento na direção $y$. Na direção $z$ o autovalor de energia do oscilador harmônico em campo zero $\frac{1}{2} \hbar \omega$ é mudado para $\frac{1}{2} \hbar \tilde{\omega}$. No plano $x-y$ o campo magnético paralelo induz uma nova massa efetiva $m_{y}^{*}$ para a componente $y$ do movimento eletrônico. Portanto, o contorno de Fermi se transforma de circular em elíptico, devido ao incremento da massa efetiva ciclotrônica em comparação com a forma circular original, razão pela qual a massa ciclotrônica é determinada, na aproximação semiclássica, pela superfície de Fermi $A_{F}$ limitada pelo contorno de Fermi $m_{c}=\frac{\hbar^{2}}{2 \pi} d A_{F} / d E$. As trajetórias eletrônicas correspondentes, em campo magnético paralelo deverão ter também a forma elíptica. Porém, em heterojunções reais de $G a A s / A l_{x} G a_{1-x} A s$ o potencial de confinamento não é harmônico e o espectro de energia precisa ser obtido através de cálculos autoconsistentes $[13,15]$.

O caso do espectro de energia de um gás bidimensional de elétrons em camadas de inversão semicondutoras, quando o campo magnético está orientado de forma paralela à camada de carga espacial, foi tratado em detalhe na referência [16]. O trabalho forneceu expressões específicas para as sub-bandas magnéticas-elétricas híbridas resultantes. Nesse trabalho foi introduzida a aproximação do potencial eletrostático $U=F_{s} z$, onde $F_{s}$ é o campo elétrico superficial junto com as condições $\vec{F}_{s} \| \vec{z}$, e $\vec{B} \| \vec{x}$, partindo das equações (1.15) e (1.16), através da introdução do ansatz $\Psi(x, y, z)=D(z) \exp \left(i k_{x} x+i k_{y} y\right)$, mostrou-se que os autovalores de energia estão dados por:

$$
\frac{E}{\hbar \omega_{c}}=\frac{k_{x}^{2} l_{B}^{2}}{2}-\frac{k_{D}^{2} l_{B}^{2}}{2}+\left(\nu_{i}+\frac{1}{2}\right)+\left(k_{D} l_{B}\right)\left(k_{y} l_{B}\right)
$$

onde $D(z)$ descreve o movimento ao longo da direção $z ; k_{x}$ e $k_{y}$ são os vetores de onda ao longo das direções $x$ e $y$, respetivamente; $z_{0}=l_{B}^{2}\left(k_{y}-k_{D}\right)$ é o centro de coordenadas do movimento, $l_{B}=\left(\frac{\hbar}{e B}\right)^{\frac{1}{2}}$ é o raio do ciclotron; $k_{D}=\frac{m F}{\hbar B}$ é o vetor de onda da velocidade de arrastamento; $\nu_{i}$ é um índice que, no caso geral, é uma função do centro de coordenadas $z_{0} / l_{B}$ e do índice da sub-banda $i$; é a constante reduzida de Planck e $\omega_{c}$ é a frequência do ciclotron.

O parâmetro $k_{D} l_{B}$ é introduzido como uma medida da razão da energia eletrostática $e F_{s} l_{B}$ com relação à energia do ciclotron $\hbar \omega_{c}$. Este parâmetro também pode ser expresso como função dos comprimentos característicos do sistema:

$$
k_{D} l_{B}=\frac{e F_{s} l_{B}}{\hbar \omega_{c}}=\frac{1}{2}\left(\frac{l_{B}}{L}\right)^{3}
$$

onde, $L=\left(\frac{\hbar^{2}}{2 m^{*} e F_{s}}\right)^{\frac{1}{3}}$ é o valor promédio da largura da camada eletrônica bidimensional. 
A Fig.(1.4), obtida da referência [16], mostra a estrutura de bandas superficiais híbridas, elétricas e magnéticas para uma camada de inversão em um semicondutor, nos limites $(a)$ elétrico e (b) magnético, na presença de campo magnético aplicado em forma paralela à camada de inversão espacial.
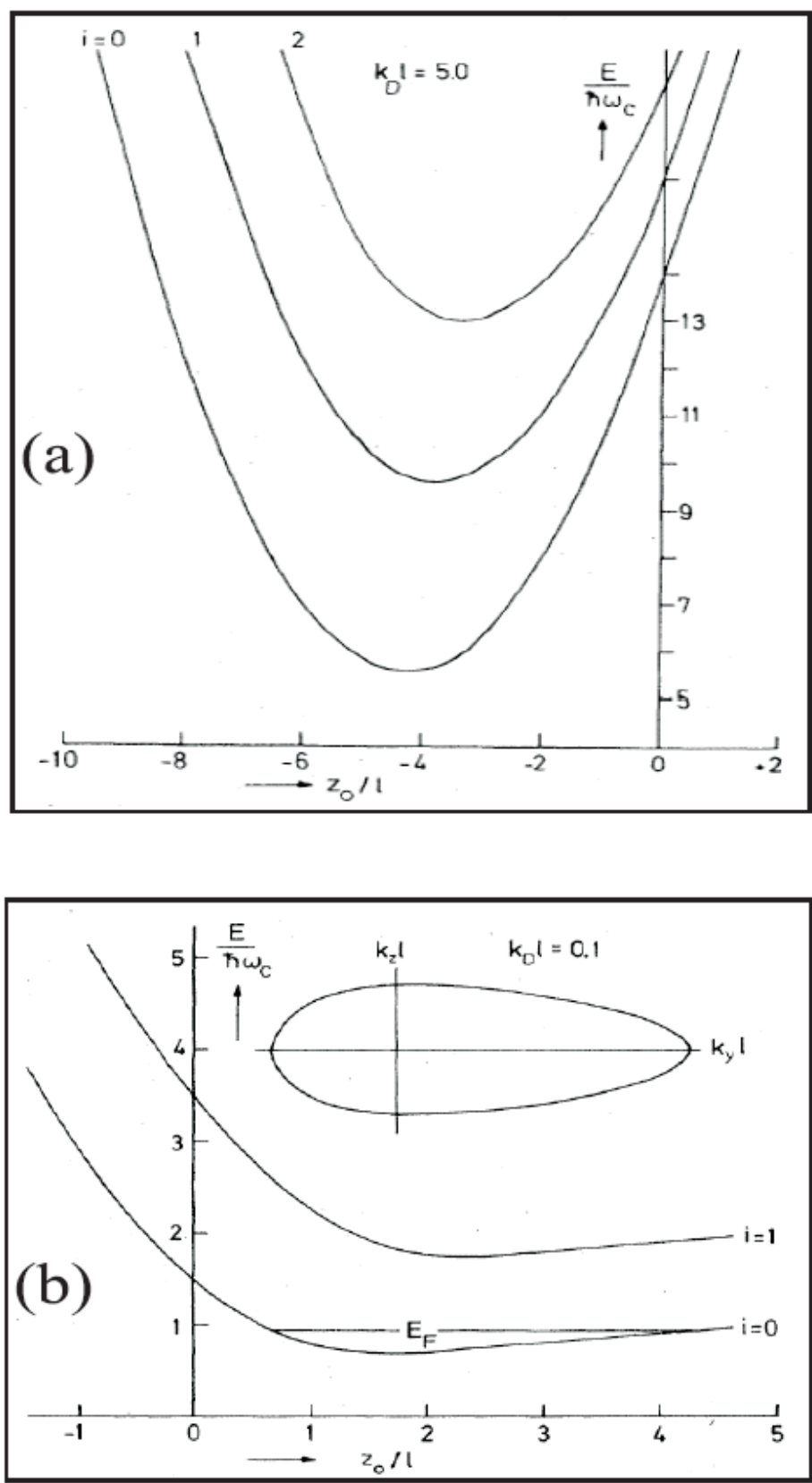

Figura 1.4: (a) Estrutura de banda de superfície $(i=0,1,2)$ no limite elétrico $\left(k_{D} l_{B} \gg 1\right)$. A ressonância ciclotrônica é somente possivel para centros de coordenadas positivos $\left(z_{0} / l \gtrsim+1\right)$. (b) Estrutura de banda de superfície $(i=0,1)$ no limite magnético $\left(k_{D} l_{B} \ll 1\right)$. A figura no extremo superior direito representa o contorno de Fermi para $E_{F}=1 / 4 \hbar \omega_{c}$. A ressonância ciclotrônica é possível para a maior parte dos centros de coordenadas. 
Quando $k_{D} l_{B} \gg+1$, a quantização elétrica é o efeito predominante, e o campo magnético conduz tanto a uma pequena anisotropia como a um deslocamento diamagnético do espectro. No caso do limite magnético, definido pela condição $k_{D} l_{B} \ll+1$, quando a energia magnética é muito maior do que a energia eletrostática, ou seja, quando a espessura da camada bidimensional é maior do que o comprimento magnético, o espectro de energia torna-se fortemente anisotrôpico e o contorno de Fermi tem uma forma oval.

Uma estimativa aproximada do parâmetro $k_{D} l_{B}$ para heteroestruturas reais de GaAs, é obtida a partir dos valores $L=41 \AA$, e $l_{B}=49 \AA$ para $B=28 \mathrm{~T}$, o qual fornece o valor $k_{D} l_{B}=0,8$. Isto implica que as quantizações eletrostáticas e magnéticas são igualmente importantes quando o campo magnético é $B=28 \mathrm{~T}$.

Uma estimativa pode ser feita para a condição de limite magnético $k_{D} l_{B} \ll+1$ através do valor $k_{D} l_{B}=0,1$, o qual pode ser alcançado somente quando o campo magnético aplicado é da ordem de $B=112 \mathrm{~T}$, que é muito maior do que os campos utilizados.

A partir dos argumentos apresentados, podemos concluir que a aplicação de um campo magnético paralelo de até $28 \mathrm{~T}$, na direção $x$, não conduz a quantização total das componentes $y$ e $z$ do momento. Portanto, o movimento no plano $x-y$ permanece contínuo, o contorno de Fermi é ligeiramente anisotrôpico, sendo ainda possível tratar a dinâmica eletrônica classicamente.

Resultados de cálculos autoconsistentes confirmam a existência de diferenças significativas entre os resultados obtidos para potenciais de confinamento parabólicos e heterojunções realísticas de $G a A s / A l_{x} G a_{1-x} A s$ [17], porém, trajetórias elípticas e ovais deverão conduzir a uma dinâmica caótica totalmente diferente em bilhares eletrônicos bidimensionais. 


\subsection{Transporte Semiclássico}

\subsubsection{Equação de Bolztmann}

Dentro do contexto semiclássico, os elétrons de condução são tratados como partículas clássicas que obedecem à estatística de Fermi-Dirac. Ao ser partículas clássicas seu comprimento de onda associada é muito menor que os tamanhos característicos das estruturas cristalinas e que as distâncias típicas da variação dos campos aplicados. Portanto, a equação de Boltzmann dá conta dos mecanismos que podem afetar à função de Distribuição de Fermi-Dirac, $f_{F D}$, em equilíbrio. Estes mecanismos podem ser de difusão, de campo externo ou de dispersão.

Uma função de distribuição de portadores de carga $f(\vec{k})$ pode ser definida como a probabilidade do estado de uma banda com energia $E_{k}$ ser ocupada por esse portador à temperatura $T$ do portador.

A equação de Boltzmann governa a variação da função $f(\vec{k})$ na presença de perturbações externas, e é expressa como [18, 19]

$$
\frac{d f(\vec{k})}{d t}=\frac{\partial f}{\partial t}+\frac{\partial f}{\partial \vec{r}} \frac{d \vec{r}}{d t}+\frac{\partial f}{\partial \vec{k}} \frac{d \vec{k}}{d t}+I_{c o l}
$$

Em particular, se o transporte ocorre no estado estacionário, ou seja, $d f(\vec{k}) / d t=0$, temos:

$$
\frac{\partial f}{\partial t}-\vec{v}(\vec{k}) \cdot \frac{\partial f}{\partial \vec{r}}+\frac{\vec{F}}{\hbar} \frac{\partial f}{\partial \vec{k}}+I_{c o l}=0
$$

onde $\vec{F}=-e(\vec{E}+\vec{v}(\vec{k}) \times \vec{H})$ é a força que atua sobre os elétrons, $\vec{v}(\vec{k})$ é a velocidade de grupo dos elétrons, e $I_{c o l}$ é o operador colisão, que expressa as mudanças no estado devido às colisões e pode ser expresso através da probabilidade de transição $W_{\text {if }}$ entre o estado inicial $i$ e o estado final $f$.

Uma vez obtida $f(\vec{k})$, estamos en condições de calcular a densidade de corrente como função de distribuição de não equilíbrio, a qual é dada por:

$$
\vec{J}=e \int d \vec{k} \vec{v}(\vec{k}) f(\vec{k})
$$




\subsubsection{Modelo de Drude}

Em 1900, P. Drude propôs o primeiro modelo para dar conta das propriedades do transporte eletrônico nos sólidos. O modelo consiste em supor que os portadores de carga (elétrons) interagem com os defeitos e as vibrações da rede, de modo que, na media realizam uma colisão cada certo intervalo de tempo ao que se chama "tempo de relaxação". Aplicando um campo elétrico $\vec{E}$, os elétrons são acelerados por uma força $\vec{F}_{e}=-e \vec{E}$, a dinâmica das partículas é descrita pela relação:

$$
m \frac{d \vec{v}}{d t}=-e \vec{E}
$$

Portanto, a velocidade eletrônica média ou velocidade de arraste $\vec{v}$, é dada por:

$$
\vec{v}(t)=\vec{v}_{0}-\frac{e t}{m} \vec{E}
$$

O modelo de Drude não esta interessado no comportamento individual dos elétrons, mas, num maior número de elétrons, é por isso que é de interesse a media da velocidade. Considerando um elétron típico em tempo zero, com $t$ sendo o tempo transcorrido desde a última colisão, a velocidade do elétron no tempo zero será $\vec{v}(0)$ imediatamente após a última colisão mais a velocidade adicional $-e t \vec{E} / m$, que posteriormente alcançou. Ao definir que o elétron emerge de uma colisão em uma direção aleatória, não existe contribuição de $\vec{v}_{0}$ à velocidade eletrônica média, a qual estará dada inteiramente por - et $\vec{E} / m$. Mas, o valor médio de $t$ é o tempo de relaxação $\tau$. Portanto temos:

$$
\vec{v}=-\frac{e \tau}{m} \vec{E}
$$

O deslocamento dos elétrons no material condutor produz uma corrente devido à velocidade de arrastamento e, portanto, dá uma densidade de corrente $\vec{J}$ igual a:

$$
\vec{J}=-e n \vec{v}
$$

Comparando com a expressão geral da lei de Ohm, a partir das Eq. (1.27) e (1.28), podemos obter uma simples relação para a condutividade elétrica:

$$
\sigma=\frac{n e^{2} \tau}{m}
$$




\subsubsection{Escalas de Comprimento Relevantes}

Pode-se distinguir entre diversas escalas de comprimento importantes em sistemas de baixa dimensionalidade. Elas são mostradas na seguinte tabela (1.1) [20]:

\begin{tabular}{|c|c|}
\hline $1 \mathrm{~mm}$ & O caminho livre médio no regime Hall quântico \\
\hline $100 \mu m$ & $\begin{array}{l}\text { O caminho livre médio/ comprimento de relaxação de } \\
\text { fase em semicondutores de alta mobilidade a } T<4 K\end{array}$ \\
\hline $\begin{array}{l}10 \mu m \\
1 \mu m\end{array}$ & Dispositivos semicondutores comerciais (1990) \\
\hline $100 \mathrm{~nm}$ & $\begin{array}{l}\text { O comprimento de onda de De Broglie em semicondu- } \\
\text { tores. O caminho livre médio em filmes metálicos poli- } \\
\text { cristalinos }\end{array}$ \\
\hline $\begin{array}{l}10 \mathrm{~nm} \\
1 \mathrm{~nm}\end{array}$ & $\begin{array}{l}\text { O comprimento de onda de De Broglie em metais. } \\
\text { Distância entre átomos. }\end{array}$ \\
\hline $1 \stackrel{\circ}{A}$ & \\
\hline
\end{tabular}

Tabela 1.1: Algumas escalas de comprimento relevantes. Notar que $1 \mu \mathrm{m}=10^{-6} \mathrm{~m}=10^{-4} \mathrm{~cm}$; $1 \mathrm{~nm}=10^{-9} \mathrm{~m}=10 \AA$

As escalas acima mencionadas têm o seguinte significado físico:

\section{O Comprimento de Onda de De Broglie, $\lambda$}

O comprimento de onda de De Broglie é definido como:

$$
\lambda=\frac{2 \pi \hbar}{p}=\frac{2 \pi}{k}
$$

onde $p(k)$ é o momento do elétron (vetor de onda). Para um gás de Fermi, o momento característico é o momento de Fermi. Para o caso de uma única sub-banda ocupada num gás bidimensional de elétrons temos:

$$
\lambda=\frac{2 \pi}{k_{F}}=\sqrt{\frac{2 \pi}{n_{s}}}
$$


onde $n_{s}$ é a densidade bidimensional de elétrons. Para o gás de Boltzmann, $p \approx \sqrt{2 m k T}$, é:

$$
\lambda=\frac{2 \pi \hbar}{\sqrt{2 m k T}}
$$

\section{O Caminho Livre Médio, $l$}

Este é um comprimento característico entre as colisões dos elétrons com as impurezas ou fônons. Ela é definida como:

$$
l=v \tau_{t r}
$$

onde $v$ é a velocidade típica e $\tau_{t r}$ é o tempo de relaxação do transporte. Ela é definida como:

$$
\frac{1}{\tau_{t r}} \propto \int d \theta \sin \theta W(\theta)(1-\cos \theta)
$$

onde $\theta$ é o ângulo de espalhamento e $W(\theta)$ é a probabilidade de espalhamento. Usualmente o transporte de elétrons é caracterizado pela mobilidade

$$
\mu_{e}=\frac{e \tau_{t r}}{m}
$$

O significado físico da mobilidade é que a velocidade de arraste alcançada pelo elétron num campo elétrico externo $\vec{E}$ é dada pela relação:

$$
v_{d}=\mu_{e} E
$$

\section{Comprimento de Relaxação de Fase, $L_{\varphi}$}

Este é especialmente um comprimento de relaxação da mecânica quântica o qual não tem análogo na física clássica. Isto é, o movimento clássico pode ser descrito como a evolução da probabilidade para achar uma partícula num ponto e tempo dado. No entanto, na mecânica quântica o estado é caracterizado pela função de onda a qual tem a fase. A fase é importante nos fenômenos de interferência, onde as funções de onda dos elétrons tendo diferentes historias, são convergidas num mesmo ponto. Se as fases das ondas não são destruídas, um fenômeno de interferência quântico específico poderá ser observado. O tempo de relaxação de fase, $\tau_{\varphi}$, describe a relaxação de memoria da fase. É claro que o espalhamento sobre qualquer potencial independente do spin estático não pode levar para o tempo de relaxação. Certamente, em qualquer potencial estacionário as equações de movimento possuem reversão-temporal. Somente os processos de 
espalhamento responsáveis pela relaxação da fase são aqueles que produzem a quebra da simetria de reversão-temporal. Dentro destes estão os espalhamentos inelásticos por fônons, colisões elétron-elétron, etc. Uma característica importante desses processos é que os elétrons sofrem muitas colisões elásticas durante o tempo $\tau_{\varphi}$. Para o movimento difusivo pode ser estimado o valor relevante de $L_{\varphi}$ através da relação:

$$
L_{\varphi}=\sqrt{D \tau_{\varphi}}
$$

onde $D=(1 / d) v l$ é a constante de difusão ( $d$ é a dimensionalidade do gás de elétrons).

\section{Comprimento de Defasagem Térmico, $L_{T}$}

O processo de relaxação anteriormente mencionado é relevante para a interferência das funções de onda pertencentes a um estado uni-eletrônico. Porém, a interferência pode ser importante para a interação de dois elétrons com energias próximas. Certamente, se a diferença de energias entre os elétrons é $\approx k T$, eles viajam quase coerentemente no decorrer do tempo $\hbar / k T$. Assim, o comprimento característico de propagação é estimado como:

$$
L_{T}=\sqrt{\frac{\hbar D}{k T}}
$$

a)

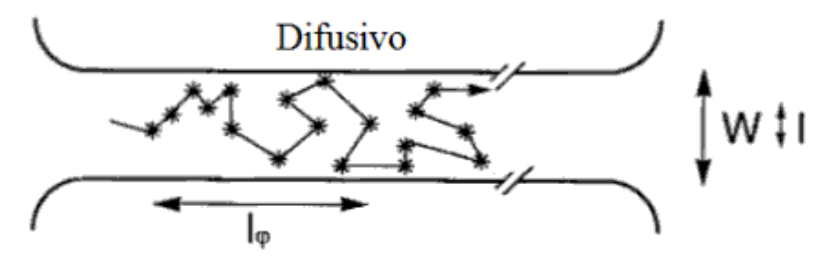

b)
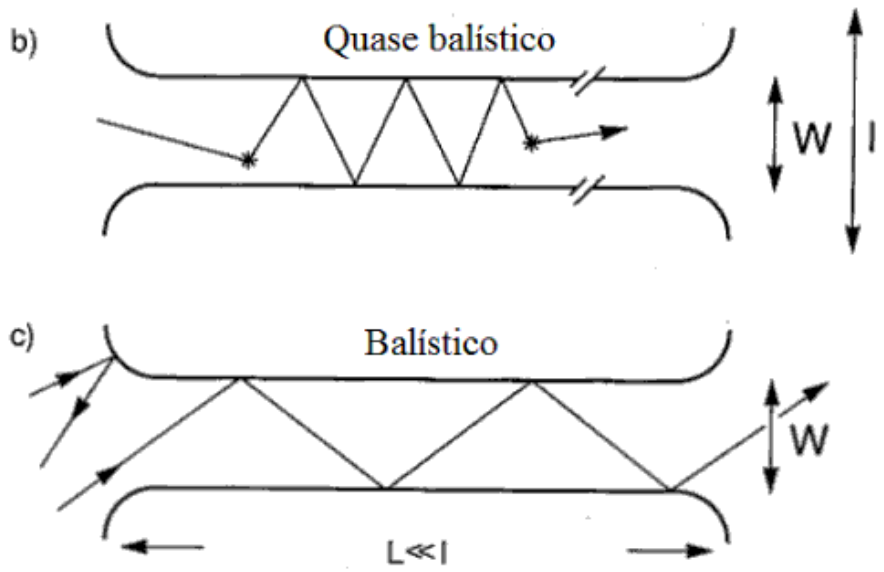

Figura 1.5: Trajetórias dos elétrons para regimes de transporte difusivo $(l<W, L)$, quase balístico $(W<l<L)$ e balístico $(l>W, L)$. 
Comparando o caminho livre médio $l$ com as dimensões características do sistema $L$, se pode discriminar entre o transporte difusivo $l \ll L$, e o transporte balístico $l \gg L, W$. Tal classificação aparece incompleta na situação onde as diferentes dimensões da amostra são substancialmente diferentes. A situação é ilustrada na Fig.(1.5) [20], para o caso onde o comprimento $L$ da amostra é muito maior que a sua largura $W$. Se a coerência de fase é levada em conta, as escalas $L_{\phi}$ e $L_{T}$ tornam-se importantes, e a situação é mais interessante. 


\subsubsection{O efeito Hall Clássico}

Para o estudo dos fenômenos de magnetotransporte, ou seja, de como a condução elétrica na presença de campo magnético é efetada, a experiência básica consiste na aplicação de corrente elétrica de baixa intensidade ao longo do sistema bidimensional de elétrons, que sofre a influência de um campo magnético, orientado perpendicularmente ao longo do gás de elétrons [21].

Suponha-se que por um semicondutor de forma retangular flui uma corrente elétrica ao longo da direção $x$ e que um campo magnético de baixa intensidade é aplicado ao longo da direção $z$, tal como se mostra na Fig.(1.6). Nesta condição um campo elétrico é produzido na direção y gerando uma diferença de potencial associada a este campo elétrico, conhecida como tensão Hall $\left(V_{H}=E_{y} l_{y}\right)$, esta tensão de Hall foi encontrada que varia proporcionalmente com a corrente e com o campo aplicado.

O efeito Hall é devido ao fluxo de uma densidade de corrente $j_{x}$, gerada por um campo elétrico $E_{x}$, de elétrons ou buracos no semicondutor na presença do campo magnético (assumiremos que os portadores de carga são elétrons). Sabe-se que uma partícula carregada num campo magnético experimenta a força de Lorentz $\vec{F}=-e \vec{v} \times \vec{B}$, onde $v$ é a velocidade de arraste dos elétrons. A direção desta força dependerá do sinal dos portadores de carga e do produto vetorial de $\vec{v}$ e $\vec{B}$, e seu efeito é o de empurrar os elétrons para o lado direito da amostra. Mas este movimento é limitado pela dimensão finita da amostra nesta direção e por isso se tem um acúmulo de elétrons nessa região. A medida que isso ocorre, o excesso de elétrons à direita e a deficiência à esquerda geram um campo elétrico $E_{y}\left(E_{y}=E_{H}\right)$. O equilíbrio ocorre quando a força elétrica gerada pelo desequilíbrio de cargas dentro da amostra equilibra a força magnética:

$$
\vec{F}=-e(\vec{E}+\vec{v} \times \vec{B})=0
$$

Visto que $\vec{E}=\left(E_{x}, E_{y}, 0\right)$ e lembrando que $\vec{j}=n_{s} e \vec{v}$, onde $n_{s}$ é a concentração de portadores, e considerando que esta densidade de corrente é causada pelo movimento de elétrons, os quais seguindo a convenção se movimentam na direção $-x$, resolvendo a equação acima na direção $y$ obtemos:

$$
V_{H}=\frac{j_{x} l_{y}}{n_{s} e} B=-\frac{1}{n_{s} e} I B
$$




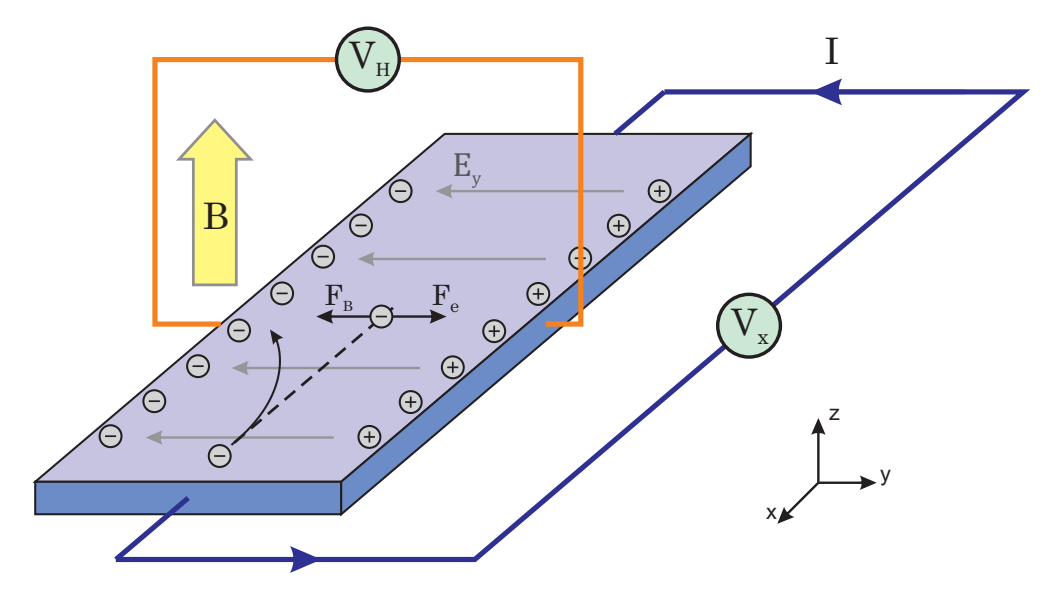

Figura 1.6: Semicondutor em forma retangular ilustrando o efeito Hall clássico.

A Eq.(1.40) também pode ser escrita como $V_{H}=I R B$, onde a magnitude $R$ chama-se coeficiente Hall:

$$
R=-\frac{1}{n_{s} e}
$$

Se os portadores de carga são buracos com concentração $p$, teríamos:

$$
R=\frac{1}{n_{s} p}
$$

Por outro lado, escrevendo a Eq.(1.40) na forma da segunda lei de Ohm, $V_{H}=I R_{H}$, obtemos:

$$
V_{H}=I\left(-\frac{B}{n_{s} e}\right)=I R_{H}
$$

Desta forma a concentração obtida a través de uma experiência de efeito Hall será dada por:

$$
n_{s}=-\frac{B}{e R_{H}} \quad\left[\mathrm{~cm}^{-2}\right]
$$

A determinação experimental de $R_{H}$ possibilita, portanto, conhecer a concentração de portadores $n_{s}$.

Outro parâmetro de transporte importante é a mobilidade de transporte dos portadores ou mobilidade Hall $\mu_{H}$, definida como a razão entre a velocidade de arrastre e o campo 
elétrico $v=\mu E$. A mobilidade pode ser obtida através da expressão:

$$
\mu_{H}=\frac{1}{e \rho_{0} n_{s}}
$$

onde $\rho_{0}$ (ou resistencia quadrado $R_{\square}$ ) é a resistividade da amostra quando $B=0$.

Para sistemas de confinamento bidimensionais, a resistividade e a condutividade são tensores de segunda ordem simbolizados respectivamente por $\hat{\rho}$ e $\hat{\sigma}$, e são inter-relacionados através da expressão $\rho=\sigma^{-1}$. Suas componentes relacionam-se por [22]:

$$
\rho_{x x}=\rho_{y y}=\frac{\sigma_{x x}}{\sigma_{x x}^{2}+\sigma_{x y}^{2}} ; \quad \rho_{x y}=\rho_{y x}=\frac{\sigma_{x y}}{\sigma_{x x}^{2}+\sigma_{x y}^{2}}
$$

sendo que $\sigma_{x x}=\sigma_{y y}$ e $\sigma_{x y}=\sigma_{y x}$. Chamamos as resistividades $\rho_{x y}$ e $\rho_{y x}$ de resistividades Hall, enquanto $\rho_{x x}$ e $\rho_{y y}$ de resistividades longitudinais. 


\subsection{Transporte Quântico}

\subsubsection{O efeito Hall quântico inteiro}

Um dos eventos mais importantes na física de semicondutores foi a descoberta da quantização da resistência de Hall por Klaus von Klitzing e M. Pepper, em 1980 [1]. Esse efeito é caracterizado por uma sequência de platôs nas medidas de resistência transversal $\left(R_{H}\right)$ ou condutividade de Hall $\left(\sigma_{H}\right)$ juntamente com o aparecimento de zeros na resistência longitudinal num gás de elétrons bidimensional (2DEG) em função do campo magnético $(B)$ aplicado. Os valores dos platôs nas medidas de resistência de Hall em função do campo aplicado podem ser expressos por:

$$
R_{H}=\frac{h}{\nu e^{2}}=\frac{1}{\nu} 25812,807 \Omega
$$

onde $h$ é a constante de Planck, e é a carga de elétron e $\nu$ é o fator de ocupação ou preenchimento, assume valores inteiros positivos $(\nu=1,2, \ldots)$. O valor da resistência no factor de preenchimento 1 é conhecido como a constante de Klitzing $\left(R_{K}=25812,807 \ldots\right)$ e é utilizado como a unidade padrão da resistência, podendo ser medido com uma precisão de até $10^{-18}$ [23, 24]. Este depende unicamente das constantes naturais $e$ e $h$ e não é afetado por qualquer parâmetro da amostra. A constante de estrutura fina $\alpha=\frac{1}{2} \mu_{0} c\left(R_{K}\right)^{-1}$, que depende da constante de permeabilidade no vácuo $\mu_{0}$, da velocidade da luz $c$ e da constante $R_{K}$, pode ser determinada pela medição da resistência de Hall quantizada [25]. Tanto o platô quantizado em $R_{H}$ e o valor da resistência zero em $R_{x x}$ pode ser entendida em termos dos níveis de Landau num sistema bidimensional.

A solução da equação de Schrödinger em duas dimensões com o campo magnético perpendicular é (Eq.(1.14)):

$$
E_{i, n, s}=E_{i}+\left(n+\frac{1}{2}\right) \hbar \omega_{c}+s g \mu_{B} B
$$

onde $E_{i}$ representa a energia da sub-banda $i=1,2, . ., n=0,1,2, \ldots$ é o número do nível de Landau e $\omega_{c}=e B / m^{*}$ é a frequência ciclotrônica.

Existem várias abordagens, formalismos e modelos sofisticados para descrever os diferentes fenômenos observados no efeito Hall quântico, mas em geral, todas estas aproximações incluem a formação dos níveis de Landau a baixas temperaturas $\left(k_{B} T \ll \hbar \omega_{c}\right)$ e a existência de estados estendidos e localizados [26, 27, 28, 29, 30]. Assim, por uma questão de simplicidade, vamos considerar o caso mais simples, onde a densidade ideal de 
estados de níveis de Landau é descrito por uma soma de funções delta $(\delta)$ :

$$
D_{\delta}(E)=\frac{e B}{h} \sum_{i, n, s} \delta\left(E-E_{i, n, s}\right)
$$

mas devido às impurezas, as funções delta são ampliadas e a densidade de estados é descrita como uma soma de gaussianas [31]:

$$
D(E)=\frac{e B}{h} \sum_{i, n, s} \frac{1}{\sqrt{2 \pi \Gamma^{2}}} \exp \left[-\frac{\left(E-E_{i, n, s}\right)^{2}}{2 \Gamma^{2}}\right]
$$

onde $\Gamma=\hbar / 2 \tau_{q}$ é o alargamento dado pelo tempo de espalhamento quântico $\tau_{q}$.

Os experimentos de magnetotransporte mostraram que entre dois níveis de Landau adjacentes a resistência de Hall tem valores fixos, no entanto, a resistência longitudinal $R_{x x}$ tem valor nulo. O fenômeno implica na existência de estados localizados nessa região. Devido à presença de impurezas e defeitos que produzem diferentes mecanismos de interação com os elétrons, o perfil da densidade de estados de um sistema bidimensional evolui de um sistema de níveis de Landau estreitos e finos (equação 1.47) para um espectro de níveis mais alargados (Eq.(1.48)), como pode ser observado na Fig.(1.7).

Nesse novo espectro de níveis existem estados localizados e estados estendidos. Os estados estendidos ou deslocalizados encontram-se muito perto do entorno do nível de Landau original, mas os estados localizados estão um pouco mais espalhados em energia, ocupando a região da cauda do nível. Somente os estados estendidos podem contribuir para a corrente elétrica em temperatura zero.

A existência de estados localizados explica o aparecimento dos platôs na resistência de Hall. Se não existissem estados eletrônicos possíveis entre os dois níveis de Landau adjacentes, o nível de Fermi poderia pular do último nível ocupado para o nível seguinte, sem a possibilidade de existência entre os dois. Assim sendo, quando o nível de Fermi se encontra dentro de uma região de existência de estados localizados, ocorre a aparição de um mínimo nas oscilações da magnetorresistência e a existência de um platô quântico na resistência de Hall. A passagem pela região de estados deslocalizados produz a depopulação de um novo nivel de Landau, aumentando assim a resistência de Hall e originando um pico nas oscilações da magnetorresistência. Em temperaturas finitas observa-se uma resistência longitudinal finita na passagem pela região de estados localizados, cuja ocorrência deve-se a processos de espalhamento "hopping" entre estados localizados no nível de Fermi. 


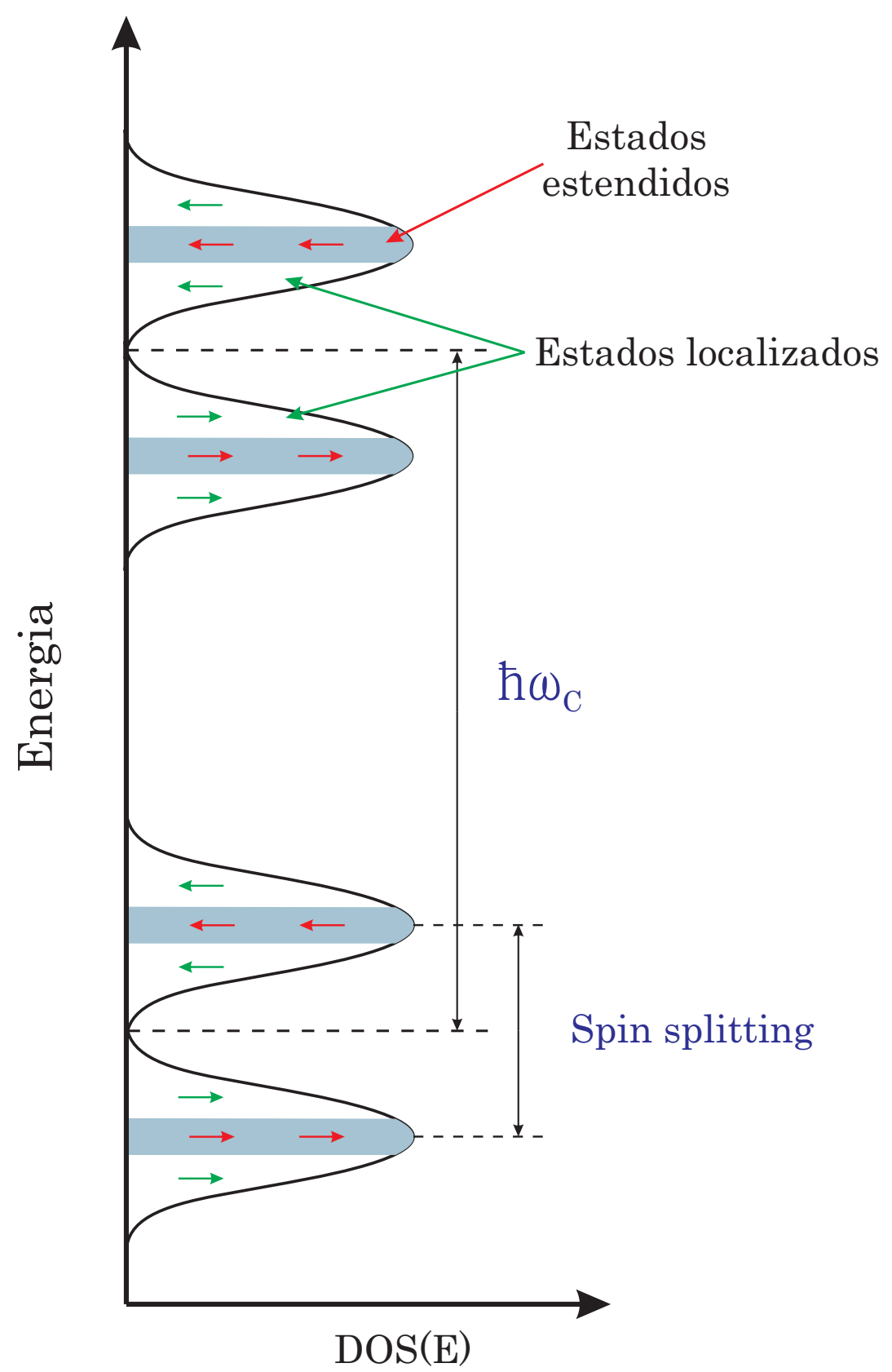

Figura 1.7: Diagrama mostrando a região de estados localizados e estados estendidos nos níveis de Landau. Também mostra-se o desdobramento Zeeman de spin.

Desse modo, $\rho_{x x}$ apresenta um comportamento oscilatório. Notemos que, dessas considerações, resulta o fato de que os zeros da resistividade longitudinal são igualmente periódicos em $1 / B$, com frequência:

$$
\Delta\left(\frac{1}{B}\right)=\frac{e(\nu+1)}{h n_{s}}-\frac{e \nu}{h n_{s}}=\frac{e}{h n_{s}}
$$

Dessa expressão se extrai experimentalmente o valor da concentração $n_{s}$. 
Na Fig.(1.8), temos um exemplo de medida experimental dos efeitos Hall clássico, Hall quântico inteiro e Shubnikov-de Haas de uma amostra, juntamente com a configuração de medição chamada barra Hall.

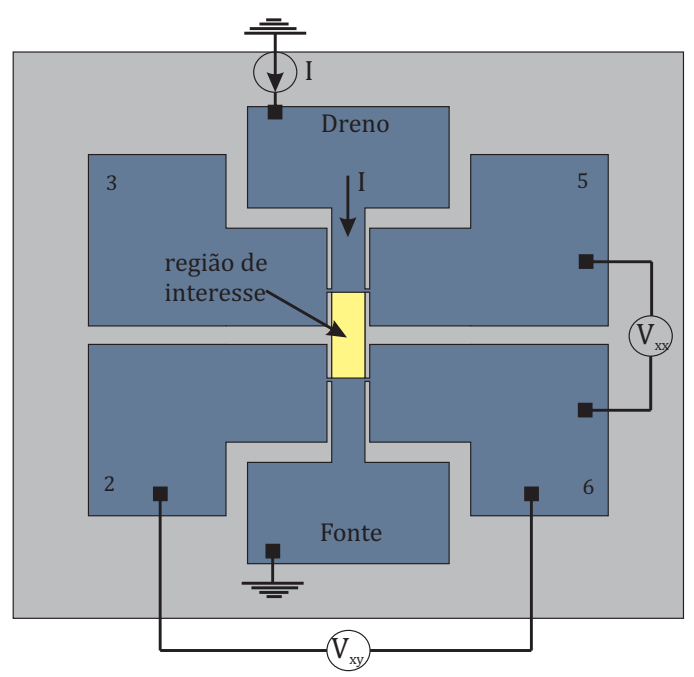

(a)

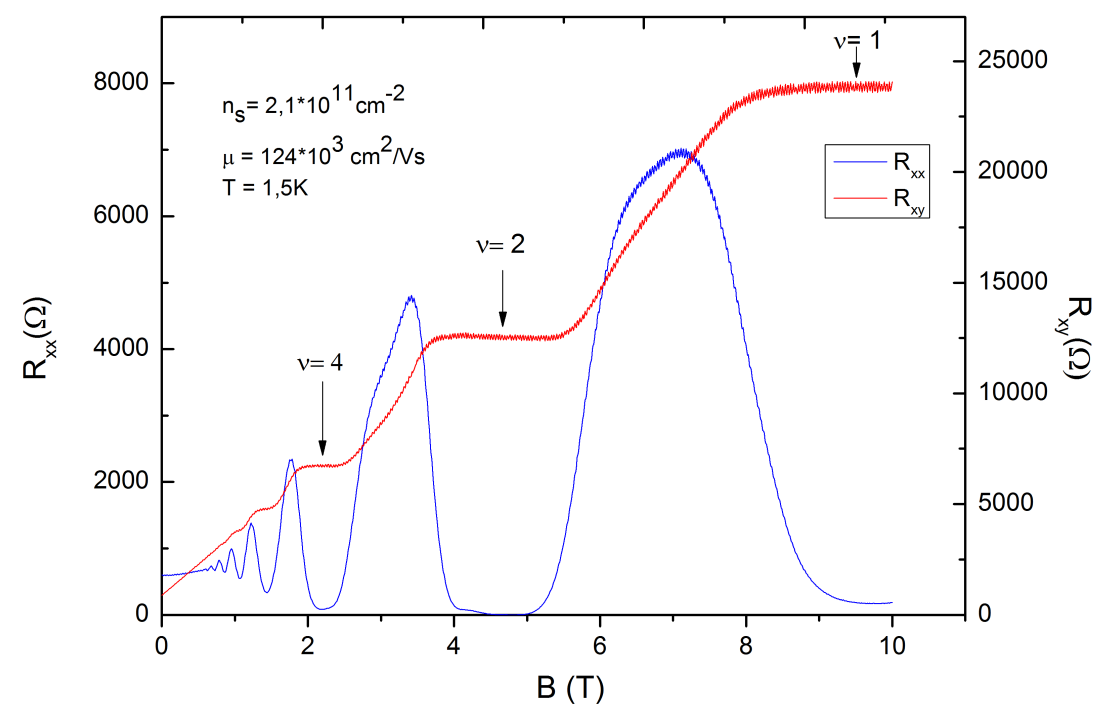

(b)

Figura 1.8: (a) Barra Hall e a configurão para medidas de $\rho_{x x}$ e $\rho_{x y}$. (b) Medidas típicas de magnetorresistência longitudinal e resistência de Hall. 


\subsubsection{O efeito Shubnikov-de Haas}

O efeito Shubnikov-de Haas ( $\mathrm{SdH}$ ) é um fenomeno de magnetotransporte observado em metais, semicondutores dopados e outros materiais submetidos à ação de campos magnéticos intensos e ultra baixas temperaturas. Estas oscilações foram descobertas em 1930 por L. Shubnikov e W.J. De Haas [32]. Este efeito é particularmente útil para o estudo de sistemas bidimensionais de elétrons por causa da grande quantidade de informações que oferece sobre os elétrons, especialmente em sistemas com varias sub-bandas ocupadas.

O fenômeno é caracterizado por oscilações da resistividade longitudinal $\left(\rho_{x x}\right)$ em função do campo magnético $B$. A resistividade longitudinal apresenta zeros em valores periódicos de $1 / B$, mas isso não ocorre para todo o domínio dos valores de campo. Para valores de campo muito baixos, temos um comportamento semiclássico em que a magnetorresistencia não apresenta oscilações [33]. Esse comportamento ocorre quando o período de uma órbita ciclotrônica é muito maior do que o tempo de espalhamento dos elétrons $\tau_{q}$ (chamado tempo quântico) [34], isto é, $\omega_{c} \tau_{q}<<1$, onde $\omega_{c}=e B / m^{*}$ é a frequência de cíclotron. Num paralelo clássico, o elétron não consegue descrever uma órbita ciclotrônica completa entre duas colisões. Entretanto, no meio termo entre o comportamento semiclássico para campos muito baixos e a região de manifestação do efeito Hall quântico para altos campos, temos um terceiro comportamento na região em que $\omega_{c} \tau_{q}>>1$. Aparece um comportamento oscilatório muito regular, também de frequência $\Delta(1 / B)=e /\left(h n_{s}\right)$, mas sem que a resistividade longitudinal se anule. Ando [35] obteve uma expressão da resistividade $\rho_{x x}(B)$ para a ocupação de apenas uma sub-banda:

$$
\rho_{x x}(B)=\rho_{x x}(0)\left[1+4 \exp \left(\frac{-\pi}{\omega_{c} \tau_{q}}\right) \frac{\xi}{\sinh \xi} \cos \left(\frac{2 \pi \Delta E}{\hbar \omega_{c}}\right)\right]
$$

onde $\rho_{x x}(0)$ é a resistividade a campo zero, $\xi=2 \pi^{2} k_{B} T / \hbar \omega_{c}, \Delta E=\left(E_{F}-E_{1}\right), T$ a temperatura e $k_{B}$ a constante de Boltzmann, enquanto a exponencial representa o alargamento dos níveis de Landau devido às colisões [36]. Mas o efeito propriamente dito é representado pela função cosseno na equação anterior. Essa expressão chama-se fórmula de Lifshitz-Kosevich, e o efeito oscilatório da resistividade é denominado Shubnikov-de Haas.

Em temperaturas da ordem de $1 \mathrm{~K}$, a população de elétrons encontra-se em estados quânticos de Landau até a energia de Fermi. Para um valor de campo magnético crescente, o número de níveis de Landau ocupados decresce cada vez que a energia de um nível se torna superior à energia de Fermi. Portanto, cada vez que se realize a condição $E_{F}=$ $(n+1 / 2) \hbar \omega_{c}$ ocorrerá a depopulação de um novo nível, provocando as oscilações no transporte, segundo a lei $1 / B$ no período. 


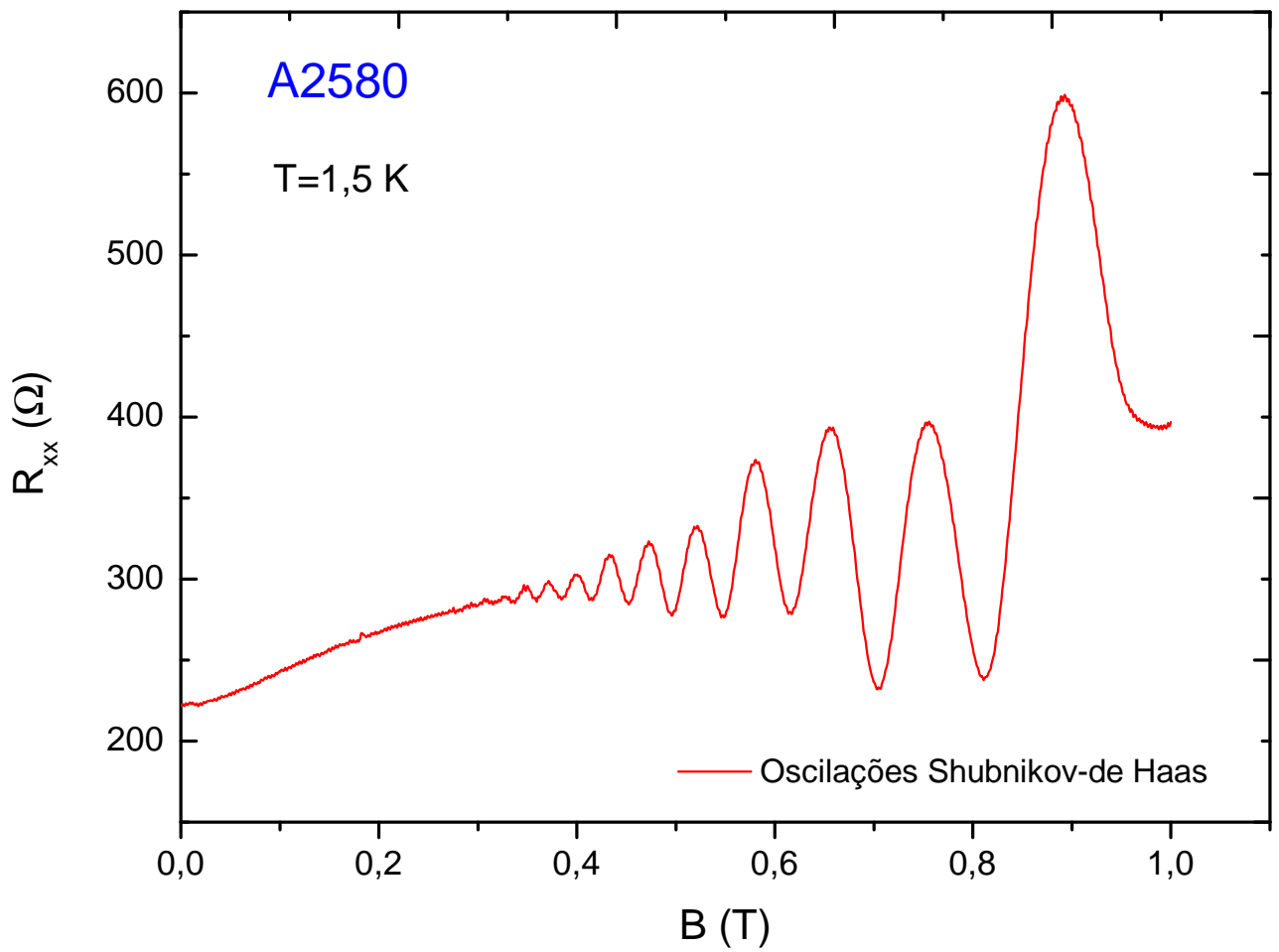

Figura 1.9: Oscilações Shubnikov-de Haas da amostra A2580 de GaAs/AlGaAs contendo um poço quântico parabólico.

O período dessas oscilações pode ser determinado se definirmos $B_{n}$ como o campo magnético no qual $E_{F}=(n+1 / 2) \hbar \omega_{c}$ (máximo em $\rho_{x x}(B)$ ), podemos reescrever essa igualdade como:

$$
\frac{1}{B_{n}}=\left(n+\frac{1}{2}\right) \frac{\hbar e}{m^{*} E_{F}}
$$

de modo que o período das oscilações entre dois máximos consecutivos é:

$$
\Delta\left(\frac{1}{B}\right)=\frac{1}{B_{n}}-\frac{1}{B_{n-1}}=\frac{\hbar e}{m^{*}} \frac{1}{E_{F}}
$$

A partir da medida do período das oscilações Shubnikov-de Haas e lembrando que $E_{F}=$ $\pi \hbar^{2} n_{s} / m^{*}$ para um gas bidimensional de elétrons, obtém-se a densidade de portadores do material $n_{s}$ :

$$
n_{s}=\frac{e}{\pi \hbar} \frac{1}{\Delta\left(\frac{1}{B}\right)}=\frac{4,835 \times 10^{10}}{\Delta\left(\frac{1}{B}\right)} \quad\left[\mathrm{cm}^{-2}\right]
$$


Quando se tem mais de uma sub-banda populada, a fórmula torna-se mais complexa, envolvendo as concentrações e tempos quânticos correspondentes. Soma-se expressões iguais à Eq.(1.50) para cada sub-banda $i$ ocupada [37]:

$$
\rho_{x x}(B)=\rho_{x x}(0)\left[1+4 \sum_{i} \exp \left(\frac{-\pi}{\omega_{i} \tau_{i}}\right) \frac{\xi_{i}}{\sinh \xi_{i}} \cos \left(\frac{2 \pi \Delta E_{i}}{\hbar \omega_{i}}\right)\right]
$$

onde se distingue genericamente a massa efetiva de cada sub-banda em $\omega_{i}=\frac{e B}{m_{i}^{*}}$.

A Fig.(1.9) mostra as oscilações Shubnikov-de Haas para uma das amostras caraterizadas no presente trabalho, contendo um poço quântico de largura $W=1000 \AA$ e densidade superficial de portadores $n_{s}=3,2 \times 10^{11} \mathrm{~cm}^{-2}$. A medição foi feita a uma temperatuda de $1,5 \mathrm{~K}$. 


\subsubsection{Transporte de estados de borda}

O efeito Hall quântico pode ser explicado pela existência de estados estendidos e localizados, como foi discutido anteriormente. Uma vez que a condutividade cai para zero, a corrente não pode fluir mais através do bulk da amostra, assim, é possível entender a resistência quantizada de Hall através de um outro modelo chamado de modelo de estados de borda.

Classicamente, os elétrons movendo-se em um campo magnético descrevem trajetórias chamadas de órbitas pulantes (skipping orbits), as quais colidem nas bordas ao longo da interface. Um esquema deste tipo de órbitas é mostrado na Fig.(1.10) [38]. Se $\vec{n}$ representa o vetor normal à interface, então os elétrons viajam com uma velocidade média na direção $\vec{n} \times \vec{B}$, assim, esses elétrons viajam em uma única direção na interface. Os estados quânticos correspondentes são os estados de borda que transportam um fluxo de corrente unidirecional.
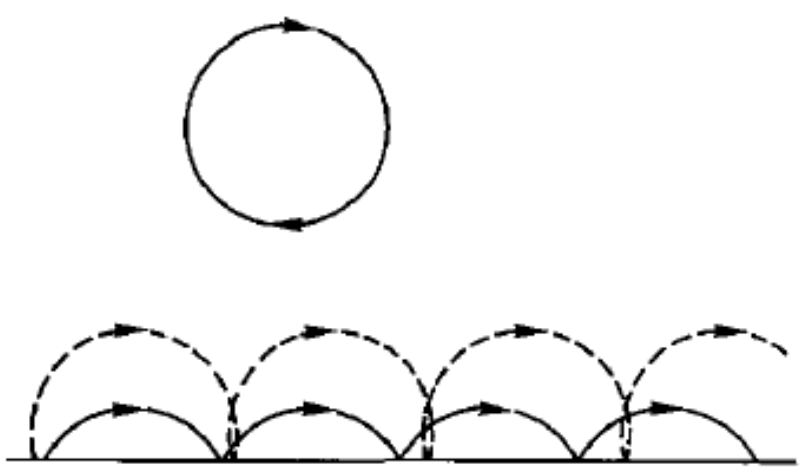

Figura 1.10: Trajetórias clássicas dos elétrons movendo-se ao longo da borda de uma amostra na presença de campo magnético.

A outra descrição do efeito Hall quântico baseada no modelo de estados de borda, foi elaborada por Marcus Büttiker [39, 40, 41]. Antes de explicar o que é um estado de borda, é necessário começar com a ideia principal do seu modelo de trasporte. Ele não é uma teoria de resposta linear com base na fórmula de Kubo, mas segue a abordagem de Landauer que baseia-se na probabilidade de transmissão e reflexão de elétrons (análogo com a propagação de ondas óptica). Assim, o formalismo de Landauer-Büttiker é baseado na ideia de descrever o transporte através de coeficientes de transmissão e reflexão [38].

Marcus Büttiker depois assumiu uma visão mais geral do efeito Hall quântico no âmbito dos estados de borda e a transmissão e reflexão de correntes de borda. Ele mostrou que 
o efeito Hall quântico pode ser explicado pela supressão do retro-espalhamento, isto é, o espalhamento de um lado da amostra para o outro, para certas regiões de fator de preenchimento em campos magnéticos intensos.

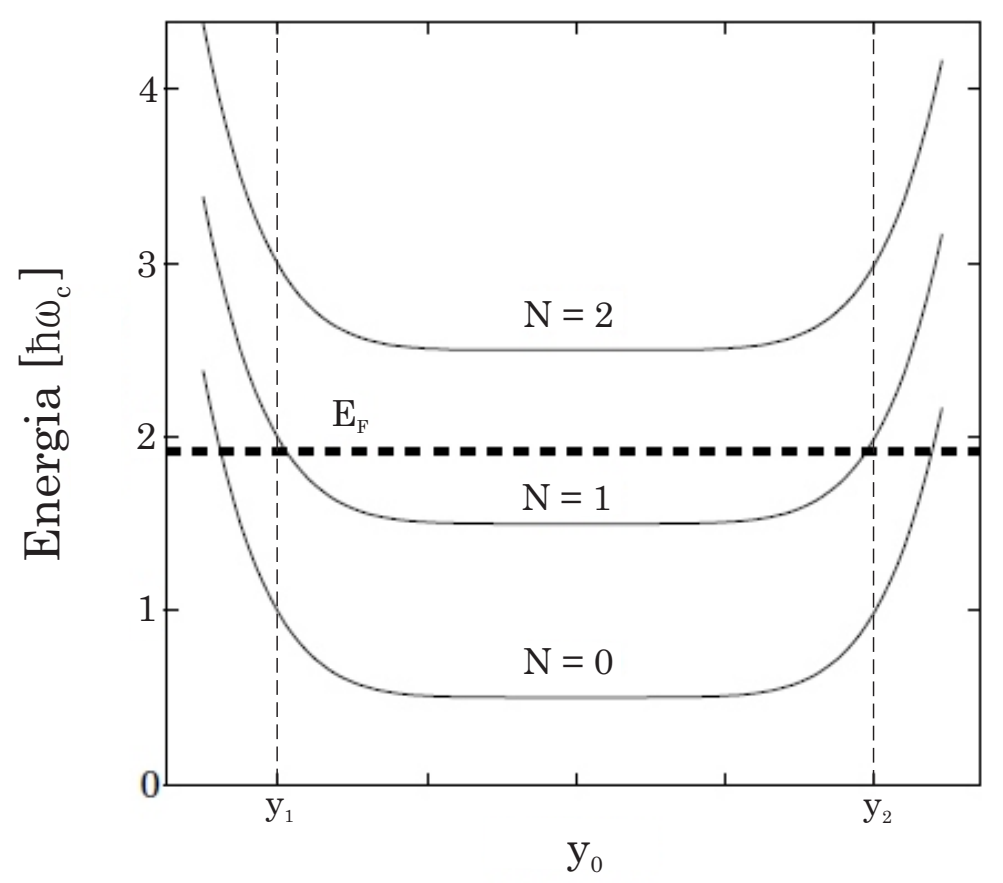

Figura 1.11: Mostra-se a dependência da posição dos espectros de energia de um 2DEG na presença de um campo magnético, em unidades da energia do cíclotron $E_{c}=\hbar \omega_{c}$. A linha pontilhada horizontal representa a energia de Fermi; neste caso existem dois niveis de Landau abaixo da $E_{F}$ no Bulk. As linhas pontilhadas verticalmente representam as posições das bordas da amostra.

Na Fig.(1.11) mostra-se os níveis de energia para vários números quânticos de Landau como uma função do parâmetro $y_{0}$ ( $y_{0}$ descreve o centro da órbita ciclotrônica) com potenciais infinitos nas bordas $y_{1}$ e $y_{2}$. Nas regiões próximas das bordas os auto-valores de energia aumentam e estados podem ser observados além das bordas da amostra definidas por $y_{1}$ e $y_{2}$.

No modelo clássico de órbitas pulantes, o centro da órbita ciclotrônica pode estar fora da amostra, e no modelo mecânico quântico o mesmo efeito é válido. No entanto, em ambos os casos, o centro de massa dos elétrons nas bordas permanece dentro da amostra, o que dá origem a um aumento na concentração de portadores perto das bordas da amostra.

Para o transporte, os estados na energia de Fermi $E_{F}$ são importantes. Em altos campos magnéticos, a densidade de estados é quantizada em níveis de Landau, os quais se curvam 
para cima nas bordas da amostra devido ao potencial de confinamento. Assim, devido ao aumento da energia dos estados perto das bordas, os estados de borda de número quântico de Landau $n$ com energias $E_{n}=\hbar \omega_{c}(n+1 / 2) \leqslant E_{F}$ são ocupados na energia de Fermi. A corrente introduzida nos estados de borda pertencentes a um número quântico de Landau é dada por:

$$
I=e v_{n, k} D(E) \Delta \mu=\frac{e}{h} \Delta \mu
$$

onde $v_{n, k}$ é a velocidade longitudinal dos estados, dada por:

$$
v_{n, k}=\frac{1}{\hbar} \frac{d E_{n, k}}{d k}=\frac{1}{\hbar} \frac{d E_{n, k}}{d y_{0}} \frac{d y_{0}}{d k}
$$

e $D(E) \Delta \mu$ é o número de estados no intervalo de energia $\Delta \mu$. Devido ao sinal diferente da inclinação da distribuição de energia dos estados nas duas bordas, a direção para a velocidade longitudinal é oposta nas duas bordas (em analogia com as órbitas pulantes clássicas nas duas bordas, Fig.(1.10)).

Os estados de borda possuem um caráter unidimensional, e a densidade de estados está, portanto, relacionada com a velocidade por:

$$
D(E)=\left(\frac{d N}{d E}\right)_{n}=\left(\frac{d N}{d k}\right)\left(\frac{d k}{d E}\right)_{n}=\frac{1}{2 \pi \hbar v_{n, k}}
$$

onde $N$ é o número de estados.

Os estados que transportam corrente pertencentes a um número quântico de Landau em uma borda são frequentemente chamados canais de borda, e são representados em diagramas bidimensionais de amostras como simples linhas com setas, onde a seta indica o sentido da velocidade longitudinal. A Fig.(1.12) mostra um exemplo do diagrama de estados de borda, ou canais de borda, em uma barra Hall com dois níveis ocupados.

O transporte em canais unidimensionais foi tratado por Büttiker. Ele estendeu a aproximação de Landauer para sistemas abertos introduzindo terminais de contato [42]. A base da sua fórmula é a conservação da corrente. Ele trata os diferentes contatos com igualdade, por exemplo, os contatos de corrente e os contatos de potenciais são equivalentes.

Como uma simplificação, Büttiker considerou somente processos elásticos na amostra, e portanto, no modelo, toda a dissipação ocorre nos contatos. A lei de conservação da corrente é aplicada a todos os contatos ao mesmo tempo. O contato fonte introduz uma 
corrente total $(I)$ na amostra, e o contato dreno remove essa corrente $(-I)$. Os contatos de potenciais não carregam nenhuma corrente $(I=0)$.

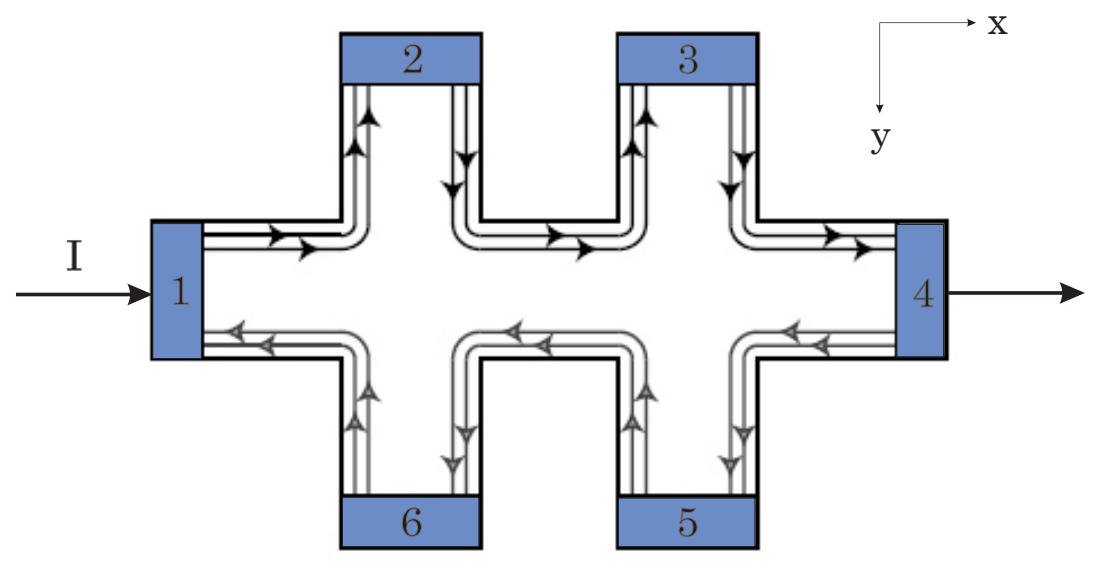

Figura 1.12: Esquema de uma barra Hall num campo magnético perpendicular com dois estados de borda $(\nu=2)$ em cada lado da amostra.

A fórmula para a corrente no contato $i$ é dada por:

$$
I_{i}=\frac{e}{h}\left[\left(M_{i}-R_{i i}\right) \mu_{i}-\sum_{j \neq i} T_{i j} \mu_{j}\right]
$$

onde $M_{i}$ é o número de canais, $R_{i i}$ é a soma dos coeficientes de reflexão do contato $i$ ao contato $i, \mu_{i}$ é o potencial eletroquímico no contato $i$, e $T_{i j}$ é a soma dos coeficientes de transmissão do contato $j$ ao contato $i$.

Através do conjunto de equações para cada contato $i$, é possível calcular todos os potenciais químicos $\mu_{i}$, e assim obter todas as resistências $R_{i j, k l}=\left(\mu_{k}-\mu_{l}\right) / e I$. Neste caso particular, a corrente é aplicada entre os terminais $i$ e $j$, e as voltagens $\mu_{k}$ e $\mu_{l}$ são detectados entre os terminais $k$ e $l$.

Para o efeito Hall quântico é assumido que os coeficientes de transmissão nas bordas para os diferentes canais de borda são iguais à unidade, e que o retro-espalhamento na amostra é suprimida. Assim, a partir da teoria de Landauer-Büttiker para o transporte quântico, espera-se que cada um desses canais contribuia à condutividade com um fator $e^{2} / h$ em ausência de retro-espalhamento. A corrente saindo de um contato é dada por $I=\Delta V M e^{2} / h$, onde $M$ é o número de canais e $\Delta V$ é a diferença de potencial entre os elétrons que entram e os elétrons que saem. 
No caso específico ilustrado na Fig.(1.12) existem dois estados de borda disponíveis, assim, quando a corrente é conduzida do contato 1 para o contato 4 temos:

Desde que a corrente $I$ está saindo do contato $1: V_{1}-V_{6}=I h / 2 e^{2}$;

Uma vez que não temos corrente nos contatos 2 e $3: V_{2}-V_{1}=0$ e $V_{3}-V_{2}=0$;

O contato 4 é o coletor da corrente, assim: $V_{4}-V_{3}=-I h / 2 e^{2}$;

Não temos corrente nos contatos 5 e $6: V_{5}-V_{4}=0$ e $V_{6}-V_{5}=0$

Resolvendo o sistema de equações lineares, obtemos:

$$
R_{x y}=\frac{V_{3}-V_{5}}{I}=\frac{h}{2 e^{2}} \quad R_{x x}=V_{2}-V_{3}=0
$$

Assim, em tais condições, a resistência longitudinal é zero e a resistência de Hall é quantizada com um valor proporcional a $h / e^{2}$ e inversamente proporcional ao número de nível de Landau. 


\subsection{O Fator $g$ de Landé em $A l_{x} G a_{1-x} A s$}

O fator $g$ de Landé de elétrons num sólido, assim como a masa efetiva, é uma propriedade intrínseca relacionada com as bandas de condução e valência do material. Devido à interação spin-órbita, o fator $g$ de um elétron na banda de condução dos semicondutores ou das heteroestruturas semicondutoras tem un valor diferente do fator $g$ de um elétron livre $(g=2,0023)$. Por exemplo, para diferentes materiais semicondutores o parâmetro $g$ assume os seguintes valores: para $G a A s$ tem-se $g=-0$, 44; para uma liga de $A l_{0,3} G a_{0,7} A s$, $g=+0,44$; na liga de $\operatorname{InP}, g=+1,2$; na liga de $\operatorname{In} S b, g=-51$ [43]; e nas heteroestruturas semicondutoras de $S i / S i_{1-x} G e_{x}$ o valor de $g$ dependerá do valor da concentração de $G e(x)$ [44]. Portanto é possível obter uma ampla variedade de valores do fator $g$ de Landé fazendo combinações adequadas de materiais semicondutores.

O modelo de 5 níveis da teoria k.p fornece uma previsão do valor do fator em semicondutores [45]. Este modelo, permite-nos determinar as bandas de energia do material semicondutor e, como consequência, o fator $g$ de Landé e a massa efetiva do portador, entre outros parâmetros. Para obter a fórmula do fator $g$ em $A l_{x} G a_{1-x} A s$ é de interesse descrever a banda de condução do GaAs na presença de um campo magnético externo. Desenvolve-se o modelo de 5 níveis perto do ponto $\Gamma$ para o caso $B \neq 0$ :

$$
\left[\frac{1}{2 m_{0}} \mathbf{P}^{2}+\mathbf{V}_{0}(r)+\frac{\hbar}{4 m_{0}^{2} c^{2}}\left(\vec{\sigma} \times \nabla V_{0}\right) \cdot \mathbf{P}+\mu_{B} \mathbf{B} \cdot \vec{\sigma}\right] \Psi=E \Psi
$$

onde $\mathbf{P}=\mathbf{p}+e \mathbf{A}$ é o momento cinético, $\mathbf{A}$ é o potencial vetor do campo magnético $\mathbf{B}$, e o termo de Pauli é escrito na notação padrão. Devido à presença do campo magnético, a equação (1.58) não é periódica. Procuramos soluções da forma:

$$
\Psi=\sum_{l} f_{l}(\mathbf{r}) \mu_{l}(\mathbf{r})
$$

onde $\mu_{l}(\mathbf{r})$ são as funções periódicas de Luttinger-Kohn [46] e $f_{l}(\mathbf{r})$ são as funções envelope que variam lentamente dentro da célula unitária. O somatório é em todas as bandas de energia. Substituindo a eq.(1.59) na eq.(1.58) é possível chegar à solução do problema de auto-valores [47].

O fator $g$ previsto pelo modelo de 5 níveis é dado pela expressão [45]:

$g_{\text {k.p }}=2+\frac{2}{3}\left[E_{P_{0}}\left(\frac{1}{E_{0}}-\frac{1}{G_{0}}\right)+E_{P_{1}}\left(\frac{1}{G_{1}}-\frac{1}{E_{1}}\right)-\frac{2 \bar{\Delta} \sqrt{E_{P_{0}} E_{P_{1}}}}{3}\left(\frac{2}{E_{1} G_{0}}+\frac{1}{E_{0} G_{1}}\right)\right]+2 C^{\prime}$ 
onde $G_{0}=E_{0}+\Delta_{0}$ e $G_{1}=E_{1}+\Delta_{1}$. Os gaps envolvidos e as energias de spinórbita são mostradas na Fig.(1.13) [45]. A constante $C^{\prime}$ representa a contribuição de bandas distantes; $\bar{\Delta}$ é a energia de spin-órbita inter-bandas. $E_{P_{0(1)}}=2 m_{0} P_{0(1)}^{2} / \hbar$ são as energias dadas em função dos elementos de matriz inter-sub-bandas $P_{0(1)}$, Em ligas de $A l_{x} G a_{(1-x)} A s$, assumimos $E_{P_{0}}, E_{P_{1}}$ e $\bar{\Delta}$ constantes [45] enquanto os outros parâmetros da equação (1.60) são funções da composição $x$.

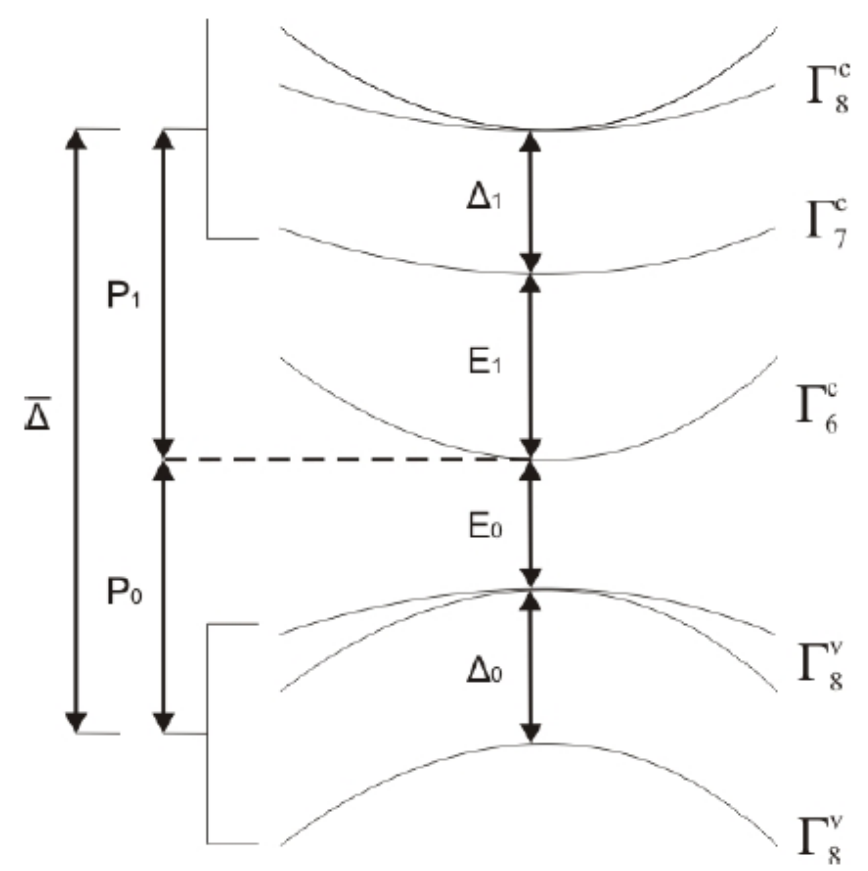

Figura 1.13: Modelo de 5 níveis para as bandas de condução e valência do GaAs perto do ponto $\Gamma$ na zona de Brillouin. Estão indicados as simetrias dos níveis em relação ao ponto $\Gamma_{i}$, os gaps de energia $E_{i}$ e as energias de spin-órbita $\Delta_{i} . P_{0}$ e $P_{1}$ são os elementos da matriz momento. $O$ zero de energia é escolhido no ponto $\Gamma_{c}^{6}$.

A dependência de $E_{0}(x)$ e $G_{0}(x)$ são conhecidas pelas referências [48, 49]:

$$
\begin{gathered}
E_{0}(x)=-\left(1,5194+1,36 x+0,22 x^{2}\right) e V \\
G_{0}(x)=-\left(1,8594+1,229 x+0,291 x^{2}\right) e V
\end{gathered}
$$

A dependência de $E_{1}(x)$ e $G_{1}(x)$ é obtida por uma interpolação linear entre os valores correspondentes para GaAs e AlAs:

$$
\begin{gathered}
E_{1}(x)=(2,969+0,971 x) e V \\
G_{1}(x)=(3,14+0,98 x) e V
\end{gathered}
$$


O parâmetro $C^{\prime}$ também varia com $x$. Porém, a comparação de resultados experimentais com a equação (1.60) mostra que seu valor é desprezível. Desse modo, a contribuição principal advém dos 5 níveis considerados pela teoria, e podemos desconsiderar $C^{\prime}$.

Na literatura encontram-se expressões empíricas para calcular $g(x)$ em $A l_{x} G a_{(1-x)} A s$. De acordo com os dados de Chadi et al [50]:

$$
g_{C h}=-0,44+4,55 x-3,5 x^{2} ; \quad(0 \leq x \leq 0,21)
$$

e resultados experimentais obtidos por Weisbuch e Hermann [51] à temperatura de $4 \mathrm{~K}$ prevêem uma depêndencia empírica dada por:

$$
g_{W H}=-0,44+4,25 x-3,9 x^{2} ; \quad(0 \leq x \leq 0,3)
$$

Essa ultima equação mostra uma boa concordância com a equação(1.60) para valores de $x$ até $30 \%$ como é mostrado na Fig.(1.14).

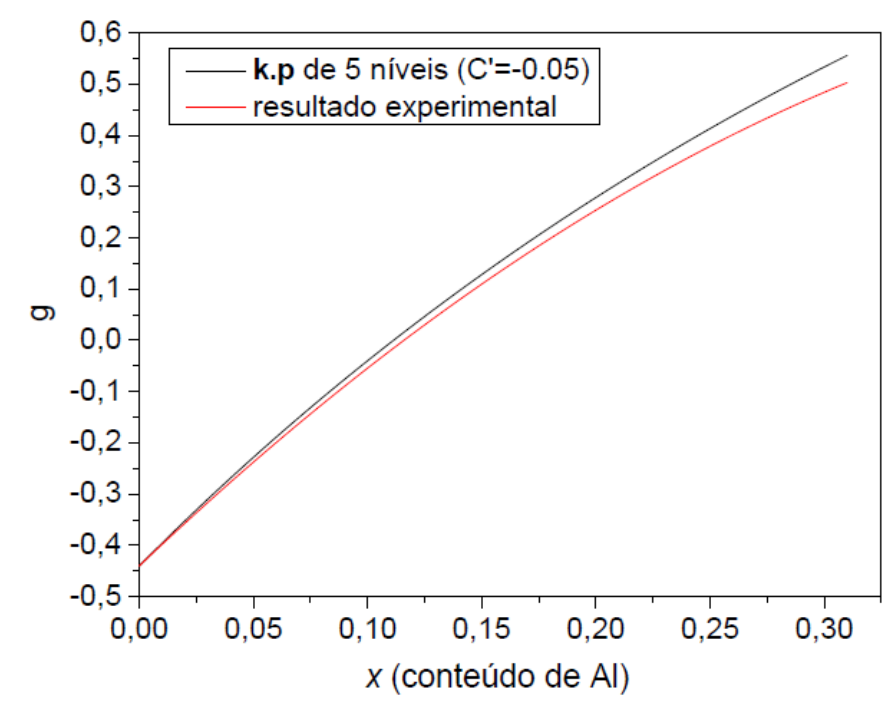

Figura 1.14: Comparação entre os valores do fator $g$ de Landé em função da concentração de Al, calculados pelo modelo de 5 bandas da teoria k.p (preto) e pela fórmula de Weisbuch e Hermann (vermelho), para a liga de $A l_{x} G a_{(1-x)} A s$. 


\subsection{Estados eletrônicos dos poços quânticos parabólicos na presença de campo magnético}

Tem sido demonstrado que os elétrons em um poço quântico parabólico ocupam várias sub-bandas elétricas. Segundo os resultados de G. Gusev et al. [14] para um poço parabólico quase cheio de largura $W=4000 \AA$ e densidade eletrônica $n_{s}=2,3 \times 10^{11} \mathrm{~cm}^{-2}$, revela a ocupação de 6 sub-bandas. Os nossos resultados de cálculos autoconsistentes (mostrados no Capítulo 2) para um poço parabólico de largura $W=2000 \AA$ e densidade eletrônica $n_{s}=3,9 \times 10^{11} \mathrm{~cm}^{-2}$ mostram a ocupação de 5 sub-bandas. O número de sub-bandas ocupadas e a largura efetiva, pode ser aumentada através do aumento da densidade superficial de elétrons.

Os poços quânticos parabólicos representam uma extensão natural de um sistema bidimensional (2D) em um sistema tridimensional (3D), quando a presença de um campo magnético aplicado, inicialmente normal ao substrato, tende a incidir paralelamente ao plano do substrato. Nesse caso, espera-se uma evolução do espectro de energia 2D para um espectro de energia 3D.

A hamiltoniana de um elétron movendo-se sob a influência de um potencial de confinamento ao longo de $z, V_{c}(z)$, e perturbado por um campo magnético paralelo orientado ao longo do eixo $x$, é dada, em forma equivalente a Eq.(1.17), por:

$$
H=-\frac{\hbar^{2}}{2 m^{*}} \frac{\partial^{2}}{\partial y^{2}}-\frac{\hbar^{2}}{2 m^{*}} \frac{\partial^{2}}{\partial z^{2}}+V_{c}(z)+\frac{m^{*} \omega_{c}^{2}}{2}\left(z+\frac{\hbar k_{x}}{e B}\right)^{2}
$$

Assim, o movimento dos elétrons na direção y é o de uma partícula livre com energia $E_{y}=\hbar^{2} k_{y}^{2} / 2 m^{*}$, e na direção $z$ observa-se o deslocamento diamagnético, com os centros das órbitas $\left(\hbar k_{x} / e B\right)$ na direção de crescimento da heteroestrutura. Portanto, o valor de $\hbar k_{x} / e B$ varia desde o centro do poço até o valor máximo $W / 2$, sendo $W$ a largura do poço quântico.

Para o caso particular de um potencial de confinamento parabólico $V_{c}(z)=\frac{1}{2} m^{*} \omega_{0}^{2} z^{2}$ na presença de um campo magnético aplicado paralelamente (ao longo do eixo $x$ ), a energia dos elétrons é dada por:

$$
E_{n}=\frac{\hbar^{2}}{2 m^{*}}\left(k_{x}^{2}+\gamma k_{y}^{2}\right)+\hbar \tilde{\omega}\left(n+\frac{1}{2}\right) ; \quad(n=0,1, \ldots)
$$

onde $\tilde{\omega}=\left(\omega_{c}^{2}+\omega_{0}^{2}\right)^{1 / 2}, \omega_{c}=e B / m^{*}, \gamma=\left(\omega_{0} / \tilde{\omega}\right)^{2}$, e $m^{*}$ é a massa efetiva. 
Para poços parabólicos largos em campos magnéticos intensos $\left(\omega_{c} \gg \omega_{0}\right)$, a Eq.(1.68) se reduz a:

$$
E_{n}=\hbar \omega_{c}\left(n+\frac{1}{2}\right)+\frac{\hbar^{2} k_{x}^{2}}{2 m^{*}}
$$

que é a expressão para o espectro de energia dos níveis de Landau 3D, onde $n$ é o número de nível de Landau.

Para um poço quântico quadrado este problema não pode ser resolvido analiticamente, porém, pode-se esperar que os resultados para ambas aproximações em campo magnético intenso não seria diferente. O espectro de energia em campo magnético paralelo é mostrado esquematicamente na Fig.(1.15) [14].

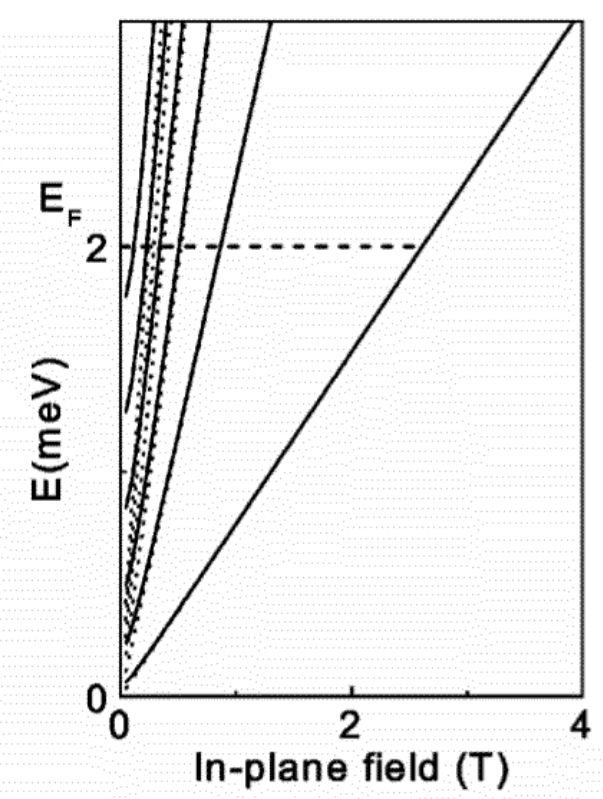

Figura 1.15: Niveis de energia de um poço quântico parabólico em função do campo magnético paralelo. A linha tracejada mostra a posição do nível de Fermi em campo magnético zero.

O comportamento é semelhante ao efeito de borda nos níveis de Landau em campo perpendicular. Aqui, porém, as energias em $k_{x}=0$ (ver Eq.1.67) correspondem às energias autoconsistentes das sub-bandas mais um termo magnético que depende quadraticamente de $z$. Isso explica por que cada curva na Fig.(1.15) inicia com as energias das sub-bandas e cresce com o incremento do campo paralelo aplicado. Também observa-se que o valor da energia de separação entre dois níveis consecutivos aumenta com o incremento do campo. Este efeito é denominado deslocamento diamagnético e é responsável pelas oscilações na resistência de um gás bidimensional de elétrons submetido a um campo paralelo. 
Espera-se que a física dos poços quânticos parabólicos largos e os sistemas tridimensionais, neste caso, devem ser semelhantes. Isto resulta do fato de que a $1 \mathrm{~T}$ o comprimento magnético $l_{B}=(\hbar / e B)^{1 / 2}$ é igual a $250 \AA$, que é menor do que a largura dos poços parabólicos largos, e um sistema eletrônico em tal campo é essencialmente tridimensional.

No caso de um campo magnético orientado ao longo do eixo $z$ (campo perpendicular), a hamiltoniana é dada por:

$$
H=-\frac{\hbar^{2}}{2 m^{*}} \frac{\partial^{2}}{\partial z^{2}}+V_{c}(z)-\frac{\hbar^{2}}{2 m^{*}} \frac{\partial^{2}}{\partial y^{2}}+\frac{m^{*} \omega_{c}^{2}}{2}\left(y-\frac{\hbar k_{x}}{e B}\right)^{2}
$$

onde na variável $z$ encontram-se as energias de confinamento do sistema $E_{i}$ ( $i$ é o índice de sub-banda) obtidas através de cálculos autoconsistentes, e ao longo de $y$ encontram-se os centros das órbitas ciclotrônicas, tendo um valor máximo na borda da amostra. Assim, o espectro de energia dos elétrons é dada por:

$$
E_{i, n}=E_{i}+\hbar \omega_{c}\left(n+\frac{1}{2}\right) ; \quad(n=0,1, \ldots)
$$

A Fig.(1.16) [14] mostra o espectro total de energia segundo a Eq.(1.71), deslocado em energia pelo valor da energia de confinamento. Claramente, esse espectro de energia é diferente do espectro de energia dos níveis de Landau 3D na presença de campo paralelo descrito pela Eq.(1.69).

Portanto, pela comparação das Eqs.(1.69) e (1.71), vê-se que o espectro de energia bidimensional (2D) é gradualmente transformado no espectro de energia tridimensional (3D), quando o campo é inclinado no sentido normal-paralelo ao plano do substrato do poço quântico parabólico. Espera-se que seja atingido o limite 3D quando o campo tende a ser paralelo e $\Delta_{i j} \rightarrow 0$, sendo $\Delta_{i j}$ as diferenças de energia entre as sub-bandas de Landau $2 \mathrm{D}$.

Em sistemas reais, os níveis de energia possuem uma largura finita $\Gamma$ devido a desordem do sistema causada por processos de espalhamento, portanto, o sistema eletrônico possui um espectro 3D de energia quando as sub-bandas eletrônicas e os seus níveis de Landau se sobrepõem, pois $\Gamma \sim \Delta_{i j}$. Assim, espera-se que todos os níveis de Landau 2D colapsem repentinamente em níveis de Landau 3D, sendo que os níveis com números quânticos elevados colapsam primeiro que os de números quânticos baixos. Atribuímos isso ao fato do alargamento dos níveis de Landau para números quânticos elevados ser maior que para números quânticos baixos. 


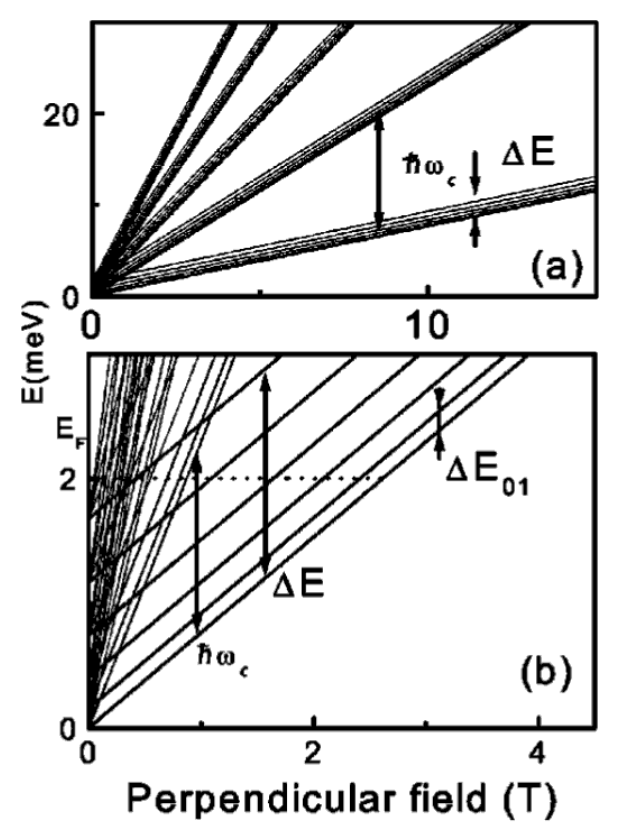

Figura 1.16: Mostra-se o espectro de energia para um poço quântico parabólico em função do campo magnético perpendicular. (a) Em campos magnéticos intensos. (b) Em campos magnéticos baixos. A posição do nível de Fermi em campo magnético zero é mostrada pela linha tracejada. As energias de ciclotron e sub-bandas são indicadas.

Quando o campo é inclinado, os efeitos acima mencionados sobrepõem-se separadamente, e o sistema assume um aspecto complexo. Mas, para o caso especial de um potencial de confinamento do tipo parabólico, $V_{c}(z)=m^{*} \omega_{0}^{2} z^{2} / 2$, soluções analíticas são encontradas [52]. Esse campo magnético inclinado, em associação com a variação do fator $g$ de Landé com respeito ao conteúdo de $A l$ na liga $A l_{x} G a_{1-x} A s$, resulta em níveis de Landau renormalizados dependentes do spin [53].

Assume-se que é alcançado um perfil de composição de $A l$ parabólico, $x(z)=a z^{2}$, onde $a$ é uma constante determinada pelo processo de crescimento. A frequência natural $\omega_{0}$ e a constante $a$, estão relacionadas pela dependência do desfasamento da banda de condução com a composição de $A l$.

Foi visto que o fator $g$ de Landé tem uma dependência com a composição de $A l$. Por exemplo, na região central de um poço quântico parabólico $(z=0)$ onde $x=0$ temos fator $g=-0,44$, valor correspondente ao GaAs puro; ao passo que nas bordas onde $x=0,29$ temos fator $g=+0,5$; e a troca de sinal acontece em $x=0,13$ onde o fator $g$ é zero. Observa-se que o fator $g$ assume valores positivos ou negativos, fato que é fundamental, já que mudanças de sinal no fator $g$ significarão mudanças de sinal na energia de Zeeman, e tal efeito pode dar origem a um efeito de "válvula de spin". 
Assim, dentro do intervalo de interesse, pode-se considerar que o fator $g$ em estruturas de $A l_{x} G a_{1-x} A s$ muda com a composição $x$ de $A l$ em forma linear [53, 54], como é mostrado na Fig.(1.17) [53].

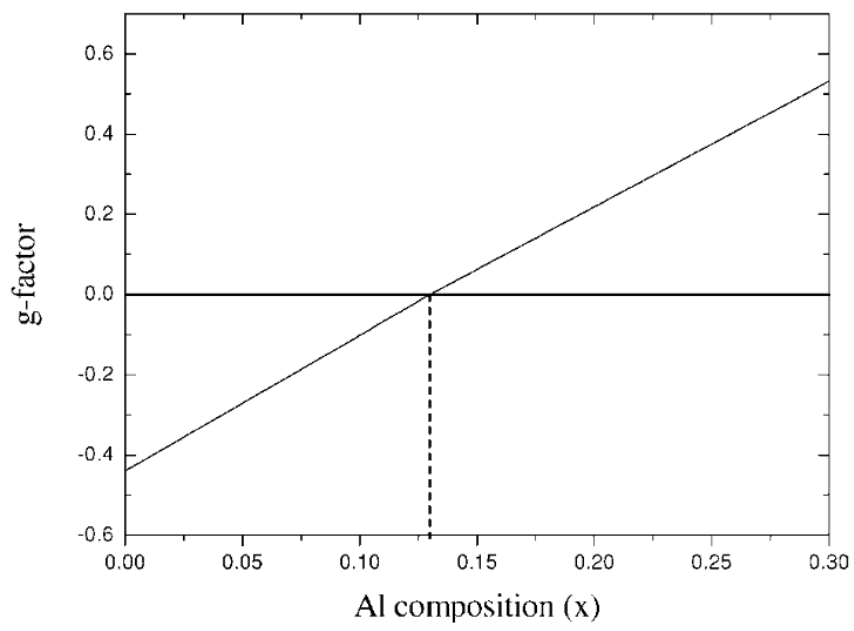

Figura 1.17: Mostra-se a dependência do fator $g$ com a composição de Al na liga $A l_{x} G a_{1-x} A s$ para $x \leq 0,3$.

A dependência linear do gator $g$ com a composição $x$ de alumínio, é dada por [53]:

$$
g(x)=-g_{0}+c x ; \quad 0 \leq x \leq 0,3
$$

onde $g_{0}=0,44$, e $c=3,24$. Considerando que a composição de $A l$ varia com $z$ da forma $x(z)=a z^{2}$, obtem-se a variação do fator $g$ ao longo do eixo $z$ :

$$
g(z)=-g_{0}+a c z^{2}
$$

A Fig.(1.18a) mostra as polarizações de spin dos estados ao longo de um poço parabólico na presença de campo paralelo. Na Fig.(1.18b) [54] mostra-se a variação do fator $g$ ao longo do eixo $z$ em um poço parabólico.

Vamos incluir a variação do fator $g$ dada pela Eq.(1.73) na hamiltoniana de um gás de elétrons confinado em um poço parabólico na presença de campo magnético inclinado $B=\left(B_{x}, 0, B_{z}\right)$. Assim, temos a hamiltoniana seguinte:

$$
H=\frac{1}{2 m^{*}}\left[\left(p_{x}+e B_{z} y\right)^{2}+p_{y}^{2}+\left(p_{z}-e B_{x} y\right)^{2}\right]+\frac{1}{2} m^{*} \omega_{0}^{2} z^{2}+g(z) \mu_{B} \vec{B} \cdot \vec{\sigma}
$$



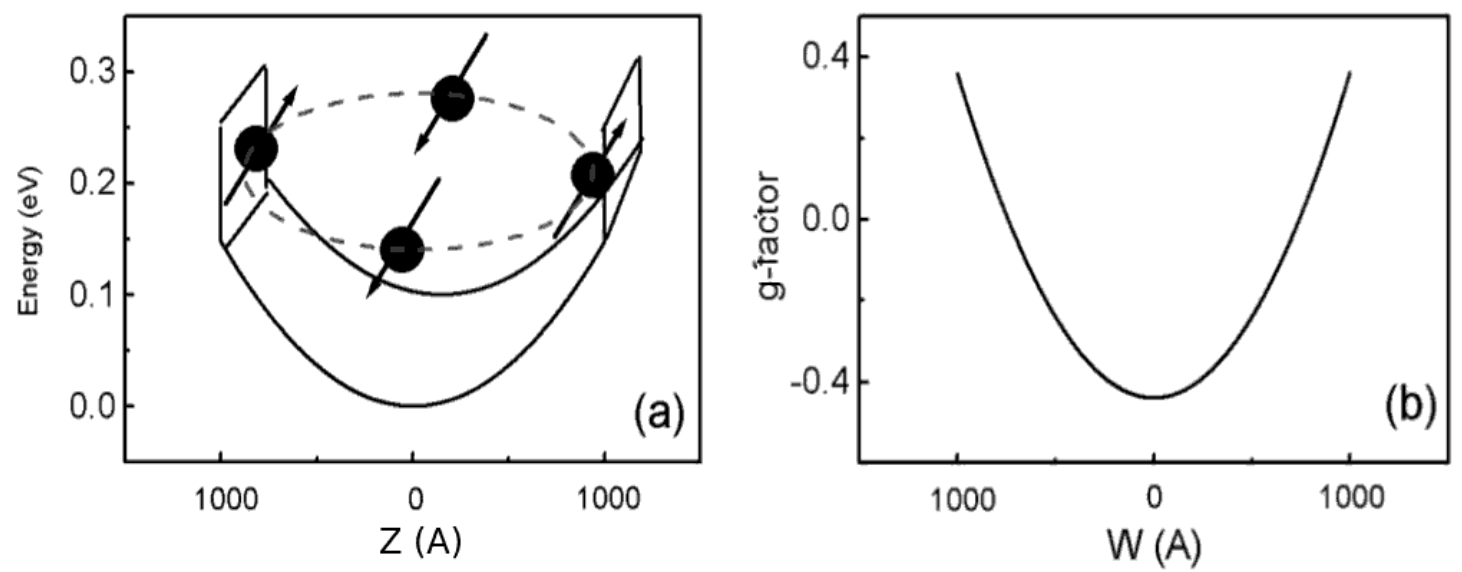

Figura 1.18: (a) Representação esquemática da orientação de spin dos elétrons em um poço quântico parabólico na presença de campo magnetico paralelo intenso. (b) Variação do fator g de Landé ao longo do eixo z.

onde $\mu_{B}$ é o magneton de Bohr, e temos usado o potencial vetor $A=y\left(-B_{z}, 0, B_{x}\right)$. Substituindo a Eq.(1.73) na Eq.(1.74) obtemos:

$$
H=\frac{1}{2 m^{*}}\left[\left(p_{x}+e B_{z} y\right)^{2}+p_{y}^{2}+\left(p_{z}-e B_{x} y\right)^{2}\right]+\frac{1}{2} m^{*} \Omega_{\sigma}^{2} z^{2}-g_{0} \mu_{B} \vec{B} \cdot \vec{\sigma}
$$

onde:

$$
\Omega_{\sigma}=\sqrt{\omega_{0}^{2}+\frac{a c \mu_{B} B}{m^{*}} \sigma} ; \quad(\sigma= \pm 1 / 2)
$$

Nota-se que nessa última expressão para a hamiltoniana, o campo magnético total acoplado ao spin eletrônico introduz uma mudança rígida, mas também aparece a frequência natural renormalizada $\Omega_{\sigma}$. Para o caso de alinhamento de spin antiparalelo $(\sigma=-1 / 2)$, o efeito é atenuar o potencial harmônico até um valor de campo magnético crítico:

$$
B_{c}=\frac{m^{*} \omega_{0}^{2}}{a c \mu_{B}}
$$

Neste campo, o elétron com a polarização de spin antiparalelo, perde o seu caráter 2D, devido a que os autoestados tornam-se níveis de Landau 3D, os quais não são afetados pela direção do campo magnético. 


\subsection{Idealização do transistor válvula de spin}

Agora, vamos apresentar a idealização do dispositivo conhecido como transistor válvula de spin de $A l G a A s / G a A s$. Através da aplicação de potenciais externos $\left(V_{g}\right)$ é possível deslocar os elétrons ao longo da direção de crescimento $(z)$, e com isso, associa-se uma mudança do fator $g$ de Landé ao longo de $z$.

Se as interações energéticas entre o spin de correntes eletrônicas e a magnetização permanente de materiais nos ofereceram dispositivos eficazes baseados na magnetoresistência gigante, podemos ter a esperança de obter êxito com dispositivos de operação similar, construídos com estruturas de $A l G a A s / G a A s$, e cujo princípio de funcionamento prescinda de materiais magnéticos, só dependendo da variação do fator $g$ de Landé.

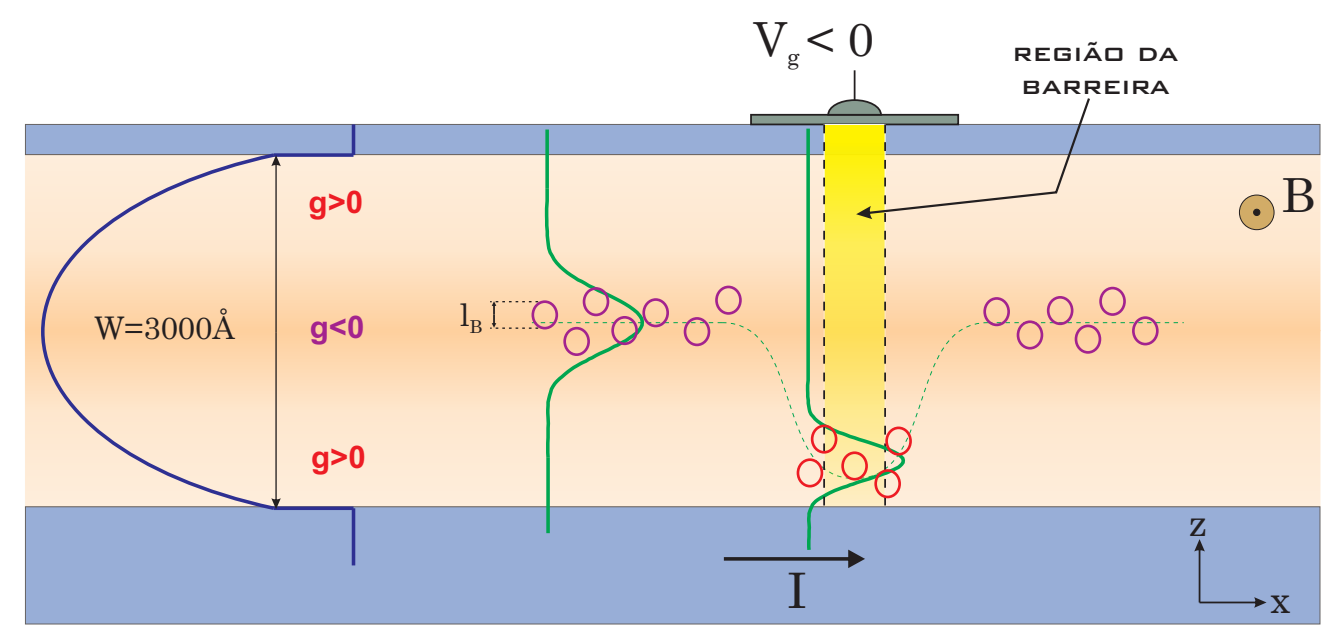

Figura 1.19: Mostra-se o esboço do transistor válvula de spin. Um fluxo de corrente I atravessa um poço quântico parabólico submetido a um campo magnético $B$ paralelo à superficie da amostra. São mostradas também as órbitas ciclotrônicas dos elétrons com raios muito menor do que a largura do poço (gás 3D de elétrons). De acordo com a localização da densidade eletrônica, o fator g será positivo ou negativo, de modo que os elétrons adquirem spin "up" (aneis roxos) ou "down" (aneis vermelhos), para que a energia de Zeeman seja mínima. A tensão de porta manipula a localização eletrônica.

Na Fig.(1.19) mostra-se o esquema para a realização de um transistor válvula de spin de AlGaAs/GaAs contendo um poço quântico parabólico. Na região central ao poço, o fator $g$ assume valores negativos, enquanto ao aproximar-se às barreiras do poço, temos uma variação gradual de $g$, e nesse intervalo assume valores negativos e positivos, sendo nas regiões proximas da barreira positivo. Mostra-se que através da região central flui uma corrente $I$ que longe da região da barreira, está associada a uma densidade eletrônica cujo centro coincide com o centro do poço, tal que o fator $g=g_{1}$ é negativo. Quando o fluxo 
de elétrons atinge a região da barreira, a ação do potencial $V_{g}$ é intensa e a função de onda é deslocada para baixo. Nessas condições, o valor do fator $g$ muda, e para tensões de porta suficientemente grandes temos que o fator $g=g_{2}$ torna-se positivo. Assim, através de diferentes mecanismos de espalhamaneto de spin (como interação spin órbita) temos uma mudança na orientação de spin dos elétrons. Essa mudança representa uma variação da energia de Zeeman $\Delta=g_{1} \mu_{B} B-g_{2} \mu_{B} B$ que, a temperaturas extremamente baixas, é maior do que $k T$ e representa portanto uma barreira de energia. O fato da barreira ser muito estreita faz com que só alguns dos elétrons deslocados pelo potencial externo, consigam mudar a sua orientação de spin, assim só esses elétrons conseguem passar através da barreira, e os outros não passam devido a que essa região resulta em uma região proibida pelo fato do fator $g$ mudar de sinal. Desse modo, espera-se que exista uma redução da corrente $I$. Assim, o dispositivo nos habilita, portanto, o controle da corrente através da aplicação de uma tensão de porta, através de um mecanismo associado ao spin.

O transistor válvula de spin e o transistor comum tem uma mesma caraterística, que em ambos utiliza-se um potencial externo modulador para controlar a corrente. A diferença básica reside no fato de que em um transistor comum esse controle baseia-se na depleção de cargas, enquanto que no transistor válvula de spin o que governa o mecanismo é a barreira de energia de Zeeman resultante da variação espacial do fator $g$ de Landé. Em acréscimo, o transistor válvula de spin precisa da ação de um campo magnético externo. 


\section{Capítulo 2}

\section{Estrutura Eletrônica dos Poços Quânticos Parabólicos de $A l_{x} G a_{1-x} A s$}

\subsection{Poços quânticos parabólicos}

O desenvolvimento dos Poços quânticos parabólicos (PQW) remotamente dopados apareceu pelo interesse no estudo das propriedades de um gás de elétrons tridimensional (3D) de alta mobilidade em campos magnéticos intensos $[8,55,56]$. A ideia original de um PQW é produzir um amplo e homogéneo gás de elétrons quase tridimensional [57]. Num sistema bidimensional de elétrons, os elétrons podem ser separados espacialmente dos doadores ionizados (Fig.(2.1a)). Isso reduz o espalhamento de Coulomb e, portanto, melhora significativamente a mobilidade dos elétrons. É evidente que uma dopagem modulada [58] não é aplicável a um sistema de elétrons que se estende indefinidamente nas tres dimensões. Não obstante, um sistema quase 3D pode ser realizado contendo duas dimensões livres e um confinamento na terceira dimensão. Se a largura do confinamento é grande em comparação com o comprimento de onda de Fermi, vários estados podem ser ocupados, e movimento ao longo do poço é possível. Nessa estrutura, os dopantes podem estar localizados fora do gás quase tridimensional de elétrons.

A Fig.(2.1b) mostra o que acontece se um poço quadrado largo é preenchido com elétrons. A interação coulombiana separa os elétrons em duas regiões estreitas cada uma com caráter bidimensional. O potencial em cada lado é equivalente à forma triangular da banda de condução de uma heteroestrutura (ver Fig.(1.1)). Só se o potencial é parabólico, como mostra a Fig.(2.1c), estas dificuldades podem ser superadas. Um potencial parabólico pode ser pensado como sendo composto por uma camada homogénea de fundo com uma densidade de carga positiva $n_{+}$. Se as interações eletron-eletron são inclusas, isto conduz a uma camada de electrões ampla, com uma densidade de electrons homogénea 3D como é ilustrado na Fig.(2.1d). 
Um poço quântico parabólico é criado através da variação gradual da composição $x$ de alumínio durante o crescimento da liga de $A l_{x} G a_{1-x} A s$ de forma a obter uma variação parabólica da banda de condução na liga [59]. Os elétrons envolvidos no processo de condução no interior do poço parabólico são provenientes das barreiras dopadas localizadas em ambos lados do poço. Uma camada de espaçamento é introduzida entre o gás confinado e as impurezas dopantes de forma a minimizar o espalhamento coulombiano dos portadores pelas impurezas ionizadas de silício. Portanto, a espessura desta camada de espaçamento é escolhida de forma a obter-se um gás de elétrons com alta mobilidade.

Para obter experimentalmente estas cargas essencialmente livres dos potenciais, temos duas possibilidades. Uma é compensar o potencial parabólico gerado por uma distribuição uniforme de cargas com um potencial parabólico confinador, criado artificialmente. A outra maneira é a dopagem remota [60] que assegura a alta mobilidade dos portadores.

a

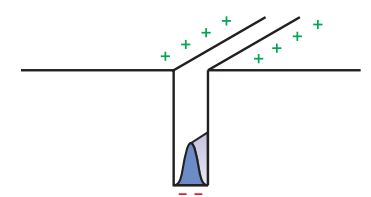

$\mathrm{b}$

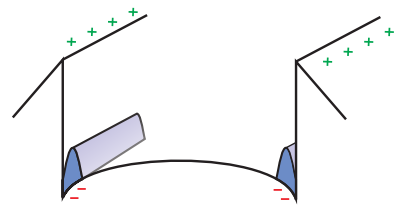

$\mathrm{c}$
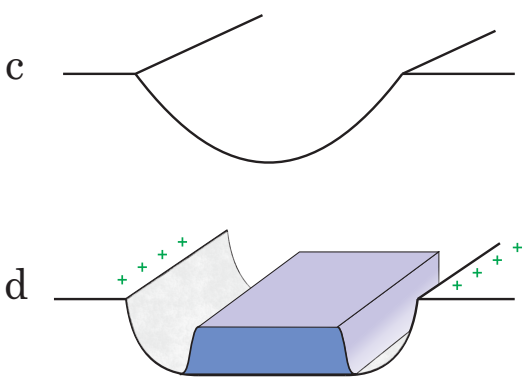

Figura 2.1: (a) Poço quântico estreito, o sistema possui caráter bidimensional. (b) Poço quântico largo, sistema separado em duas regiões cada uma com caráter bidimensional. (c) Potencial parabólico. (d) $P Q W$ com uma camada de elétrons homogénea quase tridimensional.

Na Fig.(2.2) se mostra o perfil do potencial da banda de condução em função da posição $z$, para um PQW vazio (sem cargas). Neste caso um potencial parabólico de profundidade $\Delta_{1}$ e largura $W$ é complementado por barreiras de altura $\Delta_{2}$. Para este poço, o perfil de potencial é dado por:

$$
V_{P}(z)=4 \Delta_{1}\left(\frac{z}{W}\right)^{2}
$$


Substituindo a Eq.(2.1) na equação de Poisson:

$$
\frac{d^{2} V(z)}{d z^{2}}=\frac{4 \pi e^{2}}{\epsilon} n_{3 D}(z)
$$

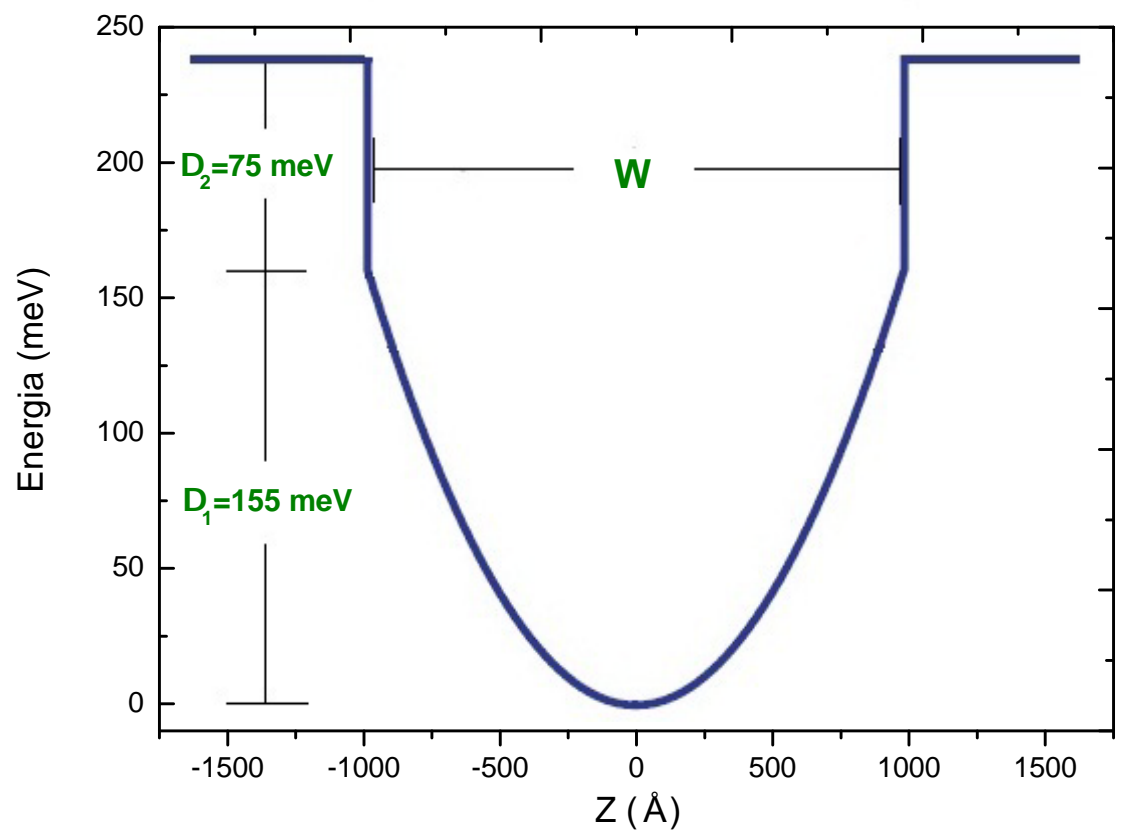

Figura 2.2: Esquema representativo que ilustra o perfil de potencial e os parâmetros relacionados com um poço parabólico. Mostra-se o caso de um poço com largura $W=2000 \AA$.

onde $V(z)$ é a energia potencial eletrostática, e é a carga do elétron, $\epsilon$ é a constante dielétrica do meio (considerada constante em toda a heteroestrutura, $\epsilon=12,6)$ e $n_{3 D}(z)$ é a densidade de carga 3D, resulta em:

$$
n_{3 D}(z)=\frac{2 \Delta_{1} \epsilon}{\pi e^{2} W^{2}}
$$

Portanto, este perfil de potencial é equivalente ao que seria produzido por uma distribuição uniforme 3D de cargas positivas. É conveniente introduzir o parâmetro $n_{+}$que descreve esta densidade de cargas positivas fictícias dada pela Eq.(2.3). Por exemplo utilizando os parâmetros do PQW mostrado na Fig.(2.2), obtém-se $n_{+}=21,5 \times 10^{15} \mathrm{~cm}^{-3}$.

Para um poço parabólico quase vazio, os níveis de energia expressam-se como os de um oscilador harmônico simples $E_{i}=(i-1 / 2) \hbar \Omega$, com frequência $\Omega^{2}=4 \pi n_{+} e^{2} / \epsilon m$ [59], com as energias das sub-bandas igualmente espaçadas entre si e com as suas funções de onda características. Entretanto, na medida que aumentamos a densidade do gás de elétrons em um poço parabólico, o potencial confinador se modifica. Desta forma, uma descrição 
detalhada do perfil do potencial e da distribuição das cargas nas sub-bandas requer de um cálculo autoconsistente, já que o potencial total depende da distribuição de cargas e a distribuição de cargas por sua vez depende do potencial confinador.

As barreiras dopadas em ambos os lados do poço, fornecem elétrons ao poço para enchelo. Quando entram no poço, os elétrons ocultam a carga positiva fictícia $n_{+}$[61]. O poço é considerado totalmente cheio quando a densidade eletrônica superficial $n_{s}$ é suficiente para blindar totalmente a carga fictícia, ou seja, $n_{s}=n_{+} W$ [55]. Para o poço da Fig.(2.2), $n_{s}^{(\text {cheio })}=4,3 \times 10^{11} \mathrm{~cm}^{-2}$. Nestas condições, o potencial total auto-consistente converge para um potencial quadrado de largura $W$, profundidade $\Delta_{2}$ e níveis de energia:

$$
E_{i}=\frac{i^{2}}{8 m}\left(\frac{h}{W}\right)^{2}
$$

onde $h$ é a constante de Planck [61].

Espera-se um poço parcialmente cheio quando a ocupação fracionária $f$, definida como:

$$
f=\frac{n_{s}}{n_{+} W}
$$

for menor do que um. Esse preenchimento parcial do poço leva a um perfil intermediário entre parabólico e quadrado. Quando o poço está parcialmente preenchido a distribuição das cargas não ocupa toda a largura do poço, desta forma, é útil considerar uma largura efetiva $W_{e}=n_{s} / n_{+}=f W$ em torno do centro do poço. A estrutura de sub-bandas do sistema é determinada através da solução autoconsistente das equações de Poisson e Schrödinger. 


\subsection{Cálculos Autoconsistentes}

Nesta seção vamos fazer cálculos teóricos do perfil de potencial da banda de condução de estruturas parabólicas seguindo o modelamento utilizado nos cálculos numéricos proposto por Rimberg e Westervelt [55]. Basicamente, estes envolvem a solução autoconsistente da equação de Schrödinger para a distribuição de cargas e a solução da equação de Poisson para o potencial eletrostático, assim podemos determinar o perfil do potencial total, os níveis de energia e a densidade de cargas no poço.

A energia do gás de elétrons em uma determinada sub-banda $i$ é a soma da energia do movimento na direção do plano $x-y$ e a energia autoconsistente $E_{i}$. A densidade de carga é construída a partir da superposição de funções de onda das diferentes subbandas eletrônicas do poço. Os níveis de energia $E_{i}$ são semelhantes aqueles do sistema do oscilador harmônico bidimensional quando $f \ll 1$, aproximando-se ao comportamento daqueles do poço quadrado quando $f \approx 1$. A equação de Schrödinger para a função de onda envelope dos elétrons na $i$-ésima sub-banda do poço $\phi_{i}(z)$, é dada por:

$$
\left[-\frac{\hbar^{2}}{2 m^{*}} \frac{d^{2}}{d z^{2}}+V(z)\right] \phi_{i}(z)=E_{i} \phi_{i}(z)
$$

onde $E_{i}$ é a energia do fundo da $i$-ésima sub-banda e $V(z)$ é o potencial autoconsistente total. O potencial total inclui os seguintes termos:

$$
V(z)=V_{p}(z)+V_{c}(z)+V_{x c}(z)
$$

onde $V_{p}(z)$ é o potencial parabólico descrito pela Eq.(2.1); $V_{c}(z)$ é o potencial eletrostático do gás de elétrons que descreve a repulsão eletrostática entre os portadores do gás e é obtido através da solução da equação de Poisson, e $V_{x c}(z)$ é o potencial de troca e correlação que leva em conta o caráter fermiônico dos elétrons na aproximação de funcional de densidade local. O potencial eletrostático é escrito como:

$$
V_{c}(z)=-\frac{2 \pi e^{2}}{\epsilon} \int_{0}^{W} n\left(z^{\prime}\right)\left|z-z^{\prime}\right| d z^{\prime}
$$

A equação de Poisson é dada pela Eq.(2.2) com

$$
n(z)=\sum_{i} n_{s}^{(i)}\left|\phi_{i}(z)\right|^{2}
$$

onde $n_{s}^{(i)}$ é a densidade da $i$-ésima sub-banda na temperatura $T=0 \mathrm{~K}$ e é dada por:

$$
n_{s}^{(i)}=\frac{m^{*}}{\pi \hbar^{2}}\left(E_{F}-E_{i}\right)
$$


O potencial de troca e correlação $V_{x c}(z)$ é incluído através de uma aproximação local para a densidade de cargas $[62,63,64]$. Mas vamos utilizar a forma proposta por Hedin e Lundqvist [65]:

$$
V_{x c}(z)=-\left[1+0,7734 x \ln \left(1+\frac{1}{x}\right)\right]\left(\frac{2}{\pi \alpha r_{s}}\right) R_{y}^{*}
$$

com:

$$
\alpha=\left(\frac{4}{9 \pi}\right)^{1 / 3}
$$

O potencial descrito é dado em unidades da constante de Rydberg definido por:

$$
R_{y}^{*}=\frac{e^{2}}{2 \epsilon a_{B}^{*}}
$$

onde $a_{B}^{*}$ é o raio de Bohr no poço parabólico, sendo expresso por:

$$
a_{B}^{*}=\frac{4 \pi \epsilon \hbar^{2}}{m^{*} e^{2}}
$$

a dependência do potencial de troca e correlação em relação à variável $z$ está em $x=r_{s} / 21$, por que $r_{s} \equiv r_{s}(z)$, a dependência em $z$ de $r_{s}$ é dada por:

$$
r_{s}(z)=\left[\frac{4}{3} \pi a_{B}^{* 3} n(z)\right]^{-1 / 3}
$$

Uma questão importante é a variação da massa efetiva $m^{*}=m^{*}(z)$ e da constante dielétrica $\epsilon=\epsilon(z)$ com a concentração de $A l$ ao longo do poço parabólico. A variação destas duas grandezas com a composição da liga $x$ é dada por [59]:

$$
\begin{gathered}
\epsilon(z)=\epsilon_{0}[12,9-2,84 x(z)] \\
m^{*}(z)=m_{0}[0,067+0,0838 x(z)]
\end{gathered}
$$

Em nosso caso usamos o valor médio $\epsilon=12,67$ empregado também por outros autores [55] e enquanto à massa efetiva vamos considerar $m^{*}=0,075 m_{0}$. Vamos desconsiderar ambas variações pois diversos trabalhos tem mostrado que estas variações não causam modificações apreciáveis na estrutura eletrônica dos poços. Por exemplo, das Sarma mostrou que uma variação da ordem de $25 \%$ na massa $m^{*}(z)$ não introduz mudanças significativas nos resultados, também mostrou que uma variação da ordem de aproximadamente $5 \%$ na constante dielétrica resulta numa variação desprezível no resultado final [66]. Em alguns cálculos é considerada uma constante dielétrica $\epsilon=12,87$ e a massa efetiva $m^{*}=0,067 m_{0}$ [67]. 
Na Fig.(2.3) [68] se mostra como referência, os resultados autoconsistentes para um PQW com $500 \AA$ de largura e $n_{s}=4,7 \times 10^{11} \mathrm{~cm}^{-2}$. O resultado desse cálculo aponta à ocupação de duas sub-bandas. Na figura podemos ver que a forma do potencial autoconsistente total (vermelho) é menos côncavo do que o potencial da banda de condução (preto), o que é causado basicamente por interações entre elétrons. Também mostra-se o potencial de repulsão coulombiana (azul) e a interação de troca e correlação (verde).

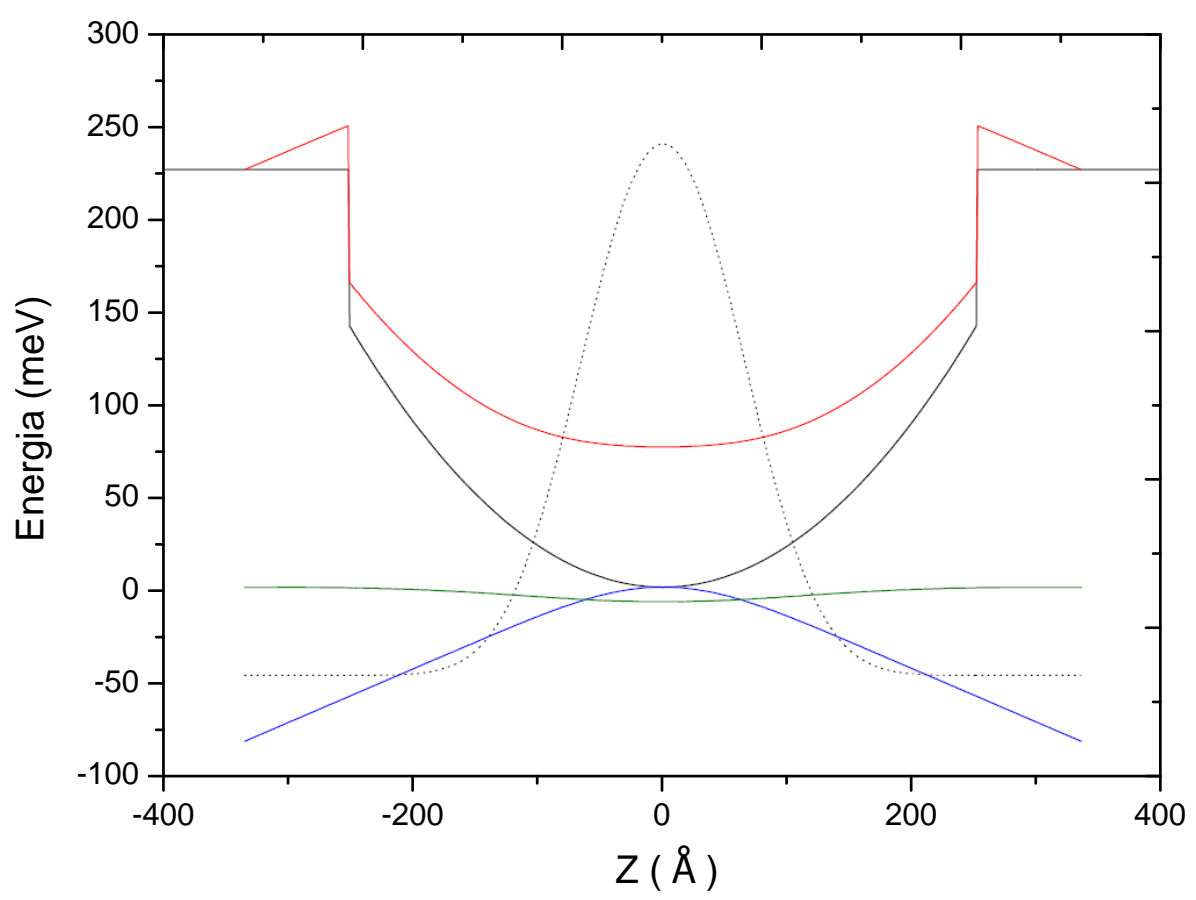

Figura 2.3: Resultados de cálculos autoconsistentes para um $P Q W$ de 500A. Mostra-se o perfil $d a$ banda de condução $V_{p}(z)$ em preto, o potencial eletrostático de repulsão coulombiana $V_{c}(z)$ em azul, e o potencial de troca e correlação $V_{x c}(z)$ em verde. O potencial autoconsistente total é mostrado em vermelho. A linha tracejada em preto é a distribuição da densidade eletrônica.

Com base na formulação teórica apresentada, se utilizou um programa computacional feito em Fortran para achar de maneira autoconsistente a estrutura de sub-bandas dos poços quânticos parabólicos, resolvendo as equações de Schrödinger e Poisson numericamente e de forma iterativa [69]. Primeiramente, um potencial inicial ou potencial de prova $V_{H}^{\text {inicial }}$ para a densidade de carga eletrônica total é calculado, o qual é somado ao potencial externo e ao potencial de troca-correlação. Com este potencial inicial resolve-se a equação de Scrödinger (Eq.(2.6)), através da qual podem ser determinadas, numa primeira iteração, as autofunções $\phi_{i}^{0}$ e os autovalores $E_{i}^{0}$. Tendo determinado as autofunções, a distribuição da densidades de carga inicial $n^{0}(z)$ também é calculada. Com estes resultados obtidos na primeira iteração, resolve-se a equação de Poisson (Eq.(2.2)) para a densidade de carga, e determina-se um novo potencial $V_{H}^{0}$. Se os potenciais de saída $V_{H}^{0}$ e de entrada $V_{H}^{\text {inicial }}$ se aproximan um do outro, dizemos que a convergência foi atingida. Se a 
convergência não foi atingida na primeira iteração, inicia-se uma segunda iteração, onde o potencial usado no cálculo será uma mistura dos dois potenciais anteriores consecutivos. A equação de mistura para os potenciais $V_{H}$ é da forma seguinte:

$$
V_{H}^{(i+1)}=V_{H}^{(i)}+\lambda\left(V_{H}^{(i+1)}-V_{H}^{(i)}\right)
$$

onde $\lambda$ é um fator independente de $z$ que varia de 0 a 1 . Para $\lambda=1$ a convergência pode ser rápida, mas também pode conduzir a uma divergência dos resultados. Valores pequenos de $\lambda$ garantem uma melhor convergência ao custo de um maior tempo de processamento.

Após a mistura de potenciais, resolve-se novamente a equação de Schrödinger, repetindose o ciclo até atingir a convergência entre os potenciais de entrada e de saída.

A seguir mostramos os resultados para diferentes poços parabólicos obtidos através do programa utilizado, e a comparação destes resultados com aqueles obtidos em outros trabalhos.

\subsection{Resultados de cálculos autoconsistentes de estru- turas parabólicas}

Com a formulação teórica apresentada determinamos a estrutura eletrônica de diferentes poços parabólicos usando os parâmetros que caracterizam ao poço. Estes cálculos nos permitem obter os níveis de energia dos estados eletrônicos, a distribuição de cargas, e o potencial total.

Apresentamos na Tabela (2.1) os resultados obtidos com o nosso programa para um poço parabólico com os seguintes parâmetros: largura do poço $W=2000 \AA$, densidade superficial de carga $n_{s}=2,0 \times 10^{11} \mathrm{~cm}^{-2}$, profundidade do poço $\Delta_{1}=155 \mathrm{meV}$ e altura da barreira $\Delta_{2}=75 \mathrm{meV}$. Estes resultados concordam muito bem com os publicados em trabalhos anteriores, como por exemplo de C. S. Sergio [70].

\begin{tabular}{|c|c|c|}
\hline Sub-banda & Energia $(\mathrm{meV})$ & $n_{s}^{(i)}\left(10^{11} \mathrm{~cm}^{-2}\right)$ \\
\hline 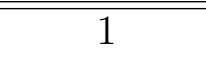 & 0,46 & 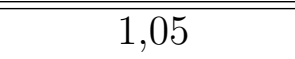 \\
\hline 2 & 1,64 & 0,71 \\
\hline 3 & 3,33 & 0,24 \\
\hline
\end{tabular}

Tabela 2.1: Energias e densidades de carga para um poço parabólico com parâmetros: $W=$ $2000 \AA, n_{s}=2,0 \times 10^{11} \mathrm{~cm}^{-2}, \Delta_{1}=155 \mathrm{meV}$ e $\Delta_{2}=75 \mathrm{meV}$. Neste poço três sub-bandas estão ocupadas. 


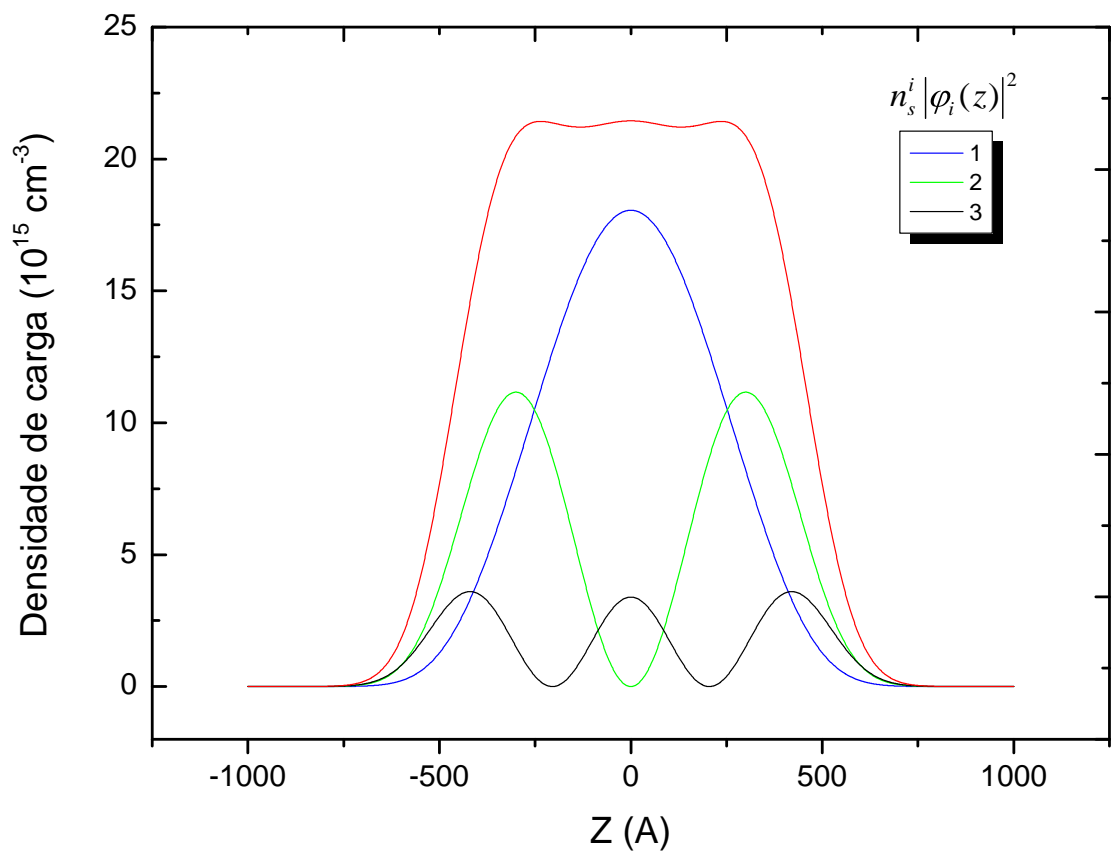

Figura 2.4: Distribuição da densidade de carga para cada uma das sub-bandas e a densidade de carga total para um poço de $W=2000 \AA$ e $n_{s}=2,0 \times 10^{11} \mathrm{~cm}^{-2}$.

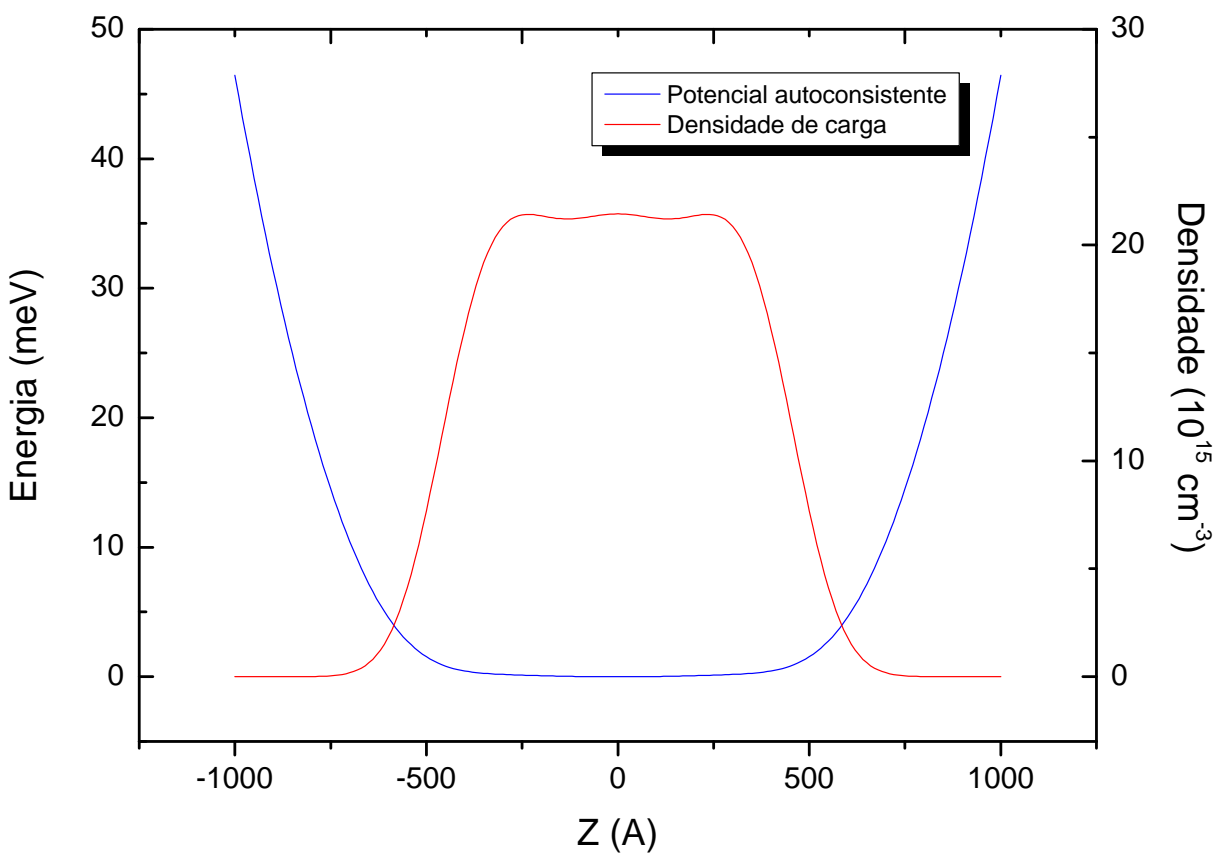

Figura 2.5: Perfil de potencial total e a densidade de carga para um poço de $W=2000 \AA e$ $n_{s}=2,0 \times 10^{11} \mathrm{~cm}^{-2}$. No gráfico do potencial não foi considerada a barreira. 
A Fig.(2.4) mostra as densidades de carga eletrônica de cada uma das sub-bandas junto com a densidade de carga total e a Fig.(2.5) mostra o perfil de potencial final convergido, simultaneamente com a densidade de carga total do sistema.

Vamos mostrar agora os resultados para um poço parabólico com os mesmos parâmetros que do poço anterior, ou seja, $W=2000 \AA, \Delta_{1}=155 \mathrm{meV}, \Delta_{2}=75 \mathrm{meV}$, mas com uma densidade de carga maior $n_{s}=3,9 \times 10^{11} \mathrm{~cm}^{-2}$. A Tabela (2.2) mostra estes resultados os quais reproduzem os resultados de A. O. de Zevallos [71].

\begin{tabular}{|c|c|c|}
\hline Sub-banda & Energia $(\mathrm{meV})$ & $n_{s}^{(i)}\left(10^{11} \mathrm{~cm}^{-2}\right)$ \\
\hline$\overline{11}$ & 0,11 & 1,14 \\
\hline 2 & 0,50 & 1,04 \\
\hline 3 & 1,15 & 0,85 \\
\hline 4 & 2,04 & 0,60 \\
\hline 5 & 3,23 & 0,27 \\
\hline
\end{tabular}

Tabela 2.2: Energias e densidades de carga para um poço parabólico com parâmetros: $W=$ $2000 \AA, n_{s}=3,9 \times 10^{11} \mathrm{~cm}^{-2}, \Delta_{1}=155 \mathrm{meV}$ e $\Delta_{2}=75 \mathrm{meV}$. Este poço tem 5 sub-bandas ocupadas.

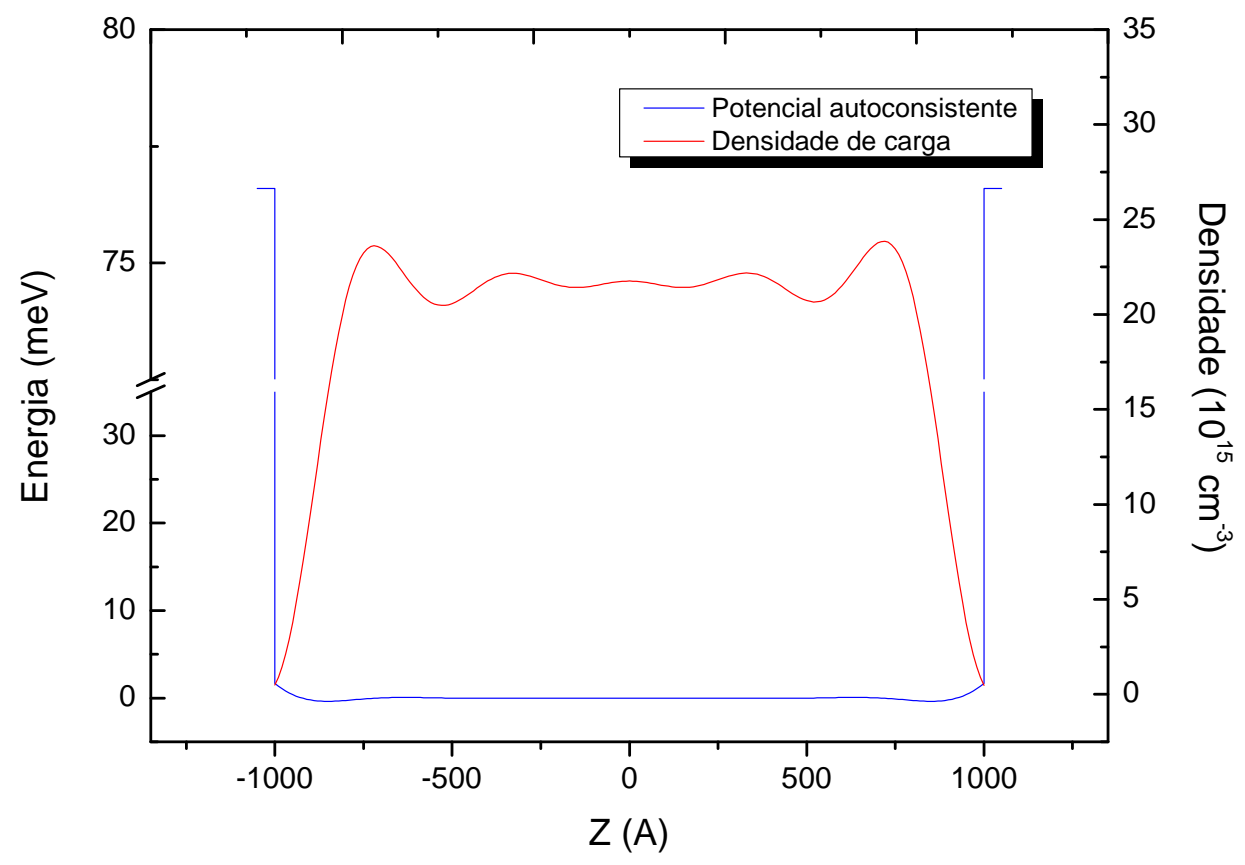

Figura 2.6: Perfil do potencial autoconsistente convergido e a densidade de carga total para um poço de $W=2000 \AA$ e $n_{s}=3,9 \times 10^{11} \mathrm{~cm}^{-2}$. 
Na Fig.(2.6) mostra-se a distribuição de densidade de carga eletrônica total para um poço de largura $W=2000 \AA$ com densidade superficial total $n_{s}=3,9 \times 10^{11} \mathrm{~cm}^{-2}$, simultaneamente com o perfil de potencial final convergido.

Também foram feitos cálculos para poços de largura menor $W=1000 \AA$. A Tabela (2.3) mostra os resultados de cálculos autoconsistentes para um poço com os parâmetros seguintes: densidade superficial de portadores $n_{s}=4,2 \times 10^{11} \mathrm{~cm}^{-2}$, profundidade do poço $\Delta_{1}=162,5 \mathrm{meV}$ e altura da barreira $\Delta_{2}=97,7 \mathrm{meV}$. Existe uma boa concordância entre os nossos resultados e os obtidos por A. O. de Zevallos [71].

\begin{tabular}{|c|c|c|}
\hline Sub-banda & Energia $(\mathrm{meV})$ & $n_{s}^{(i)}\left(10^{11} \mathrm{~cm}^{-2}\right)$ \\
\hline 1 & $\overline{1,64}$ & 2,58 \\
\hline 2 & 5,48 & 1,51 \\
\hline 3 & 10,48 & 0,10 \\
\hline
\end{tabular}

Tabela 2.3: Energias e densidades de carga para um poço parabólico com parâmetros: $W=1000$ $\AA, n_{s}=4,2 \times 10^{11} \mathrm{~cm}^{-2}, \Delta_{1}=162,5 \mathrm{meV}$ e $\Delta_{2}=97,7 \mathrm{meV}$. Neste poço três sub-bandas estão ocupadas.

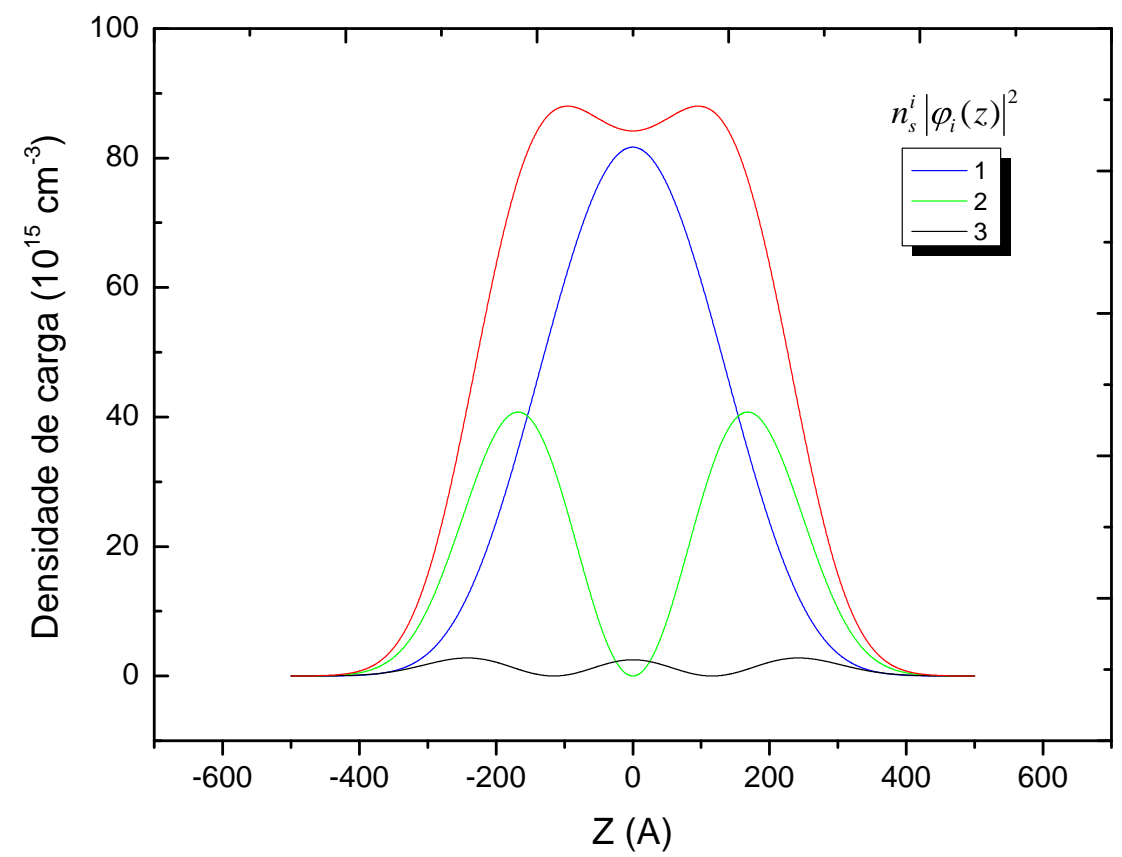

Figura 2.7: Distribuição da densidade de carga para cada uma das sub-bandas e a densidade de carga total para um poço de $W=1000 \AA$ e $n_{s}=4,2 \times 10^{11} \mathrm{~cm}^{-2}$. 
A Fig.(2.7) mostra a distribuição de densidade de carga para cada uma das sub-bandas, e também a densidade total para o poço de largura $W=1000 \AA$ e densidade superficial de carga $n_{s}=4,2 \times 10^{11} \mathrm{~cm}^{-2}$. Na Fig.(2.8) mostra-se o perfil de potencial convergido e a distribuição da densidade total.

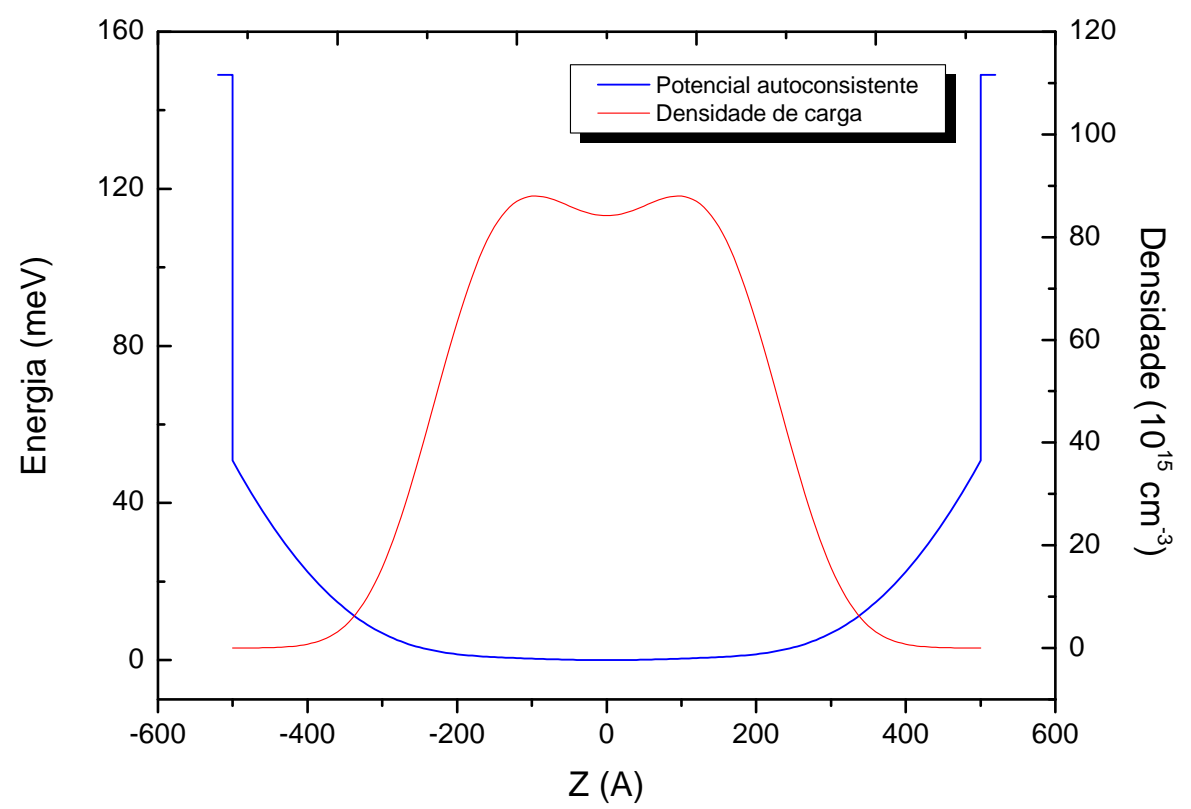

Figura 2.8: Potencial autoconsistente total e a densidade de carga total para um poço de $W=$ $1000 \AA$ e $n_{s}=4,2 \times 10^{11} \mathrm{~cm}^{-2}$.

Observa-se que para os poços parabólicos com a mesma largura $W=2000 \AA$ (Fig.(2.5) e Fig.(2.6)), o número de sub-bandas ocupadas é maior no poço com maior densidade superficial de carga, mas os níveis de energia no poço com maior densidade são mais baixos que no poço com menor densidade. Uma maior densidade de portadores no poço faz que o potencial parabólico original se modifique mais, caracterizado pelo achatamento do fundo do poço, assim para uma maior densidade, o poço tende a adotar a forma de um poço quadrado, como mostra-se na Fig.(2.6).

O poço com menor densidade $n_{s}=2,0 \times 10^{11} \mathrm{~cm}^{-2}$ tem uma ocupação fracionária $f=0,46$ (Eq. (2.5)), e a distribuição de densidade de carga ocupa uma largura efetiva $W_{e}=920 \AA$. Enquanto, o poço com densidade $n_{s}=3,9 \times 10^{11} \mathrm{~cm}^{-2}$ tem uma ocupação fracionária $f=0,90$, e a distribuição de densidade de carga ocupa uma largura efetiva $W=1800 \AA$. No caso do poço com $f=0,90$, este encontra-se quase cheio $\left(N_{s}^{\text {cheio }}=\right.$ $4,3 \times 10^{11} \mathrm{~cm}^{-2}$ ) e o potencial original modifica-se mais que no caso do poço com $f=0,46$. 


\subsection{Cálculos da estrutura de sub-bandas dos poços parabólicos empregados no trabalho}

As amostras contendo poços quânticos parabólicos foram estudadas através de medidas de magnetotransporte para determinar-se a concentração eletrônica no interior desses poços. Também foi determinada a concentração de cada uma das sub-bandas através de um análises de Fourier (FFT) das oscilações Shubnikov-de Haas. A descrição detalhada deste tipo de análises será feita mais adiante.

A seguir, são mostrados os resultados dos cálculos autoconsistentes para a determinação da estrutura de sub-bandas das amostras, empregando os valores medidos da densidade superficial de carga. Também são comparados com os resultados obtidos das medidas experimentais.

- Amostra A2578: $W=1000 \AA$ e $n_{s}=2,1 \times 10^{11} \mathrm{~cm}^{-2}$.

Para a determinação autoconsistente dos níveis de energia, perfil de potencial e distribuição de cargas desse poço de largura $W=1000 \AA$, utilizou-se a concentração eletrônica obtida das medidas de magnetotransporte, $n_{s}=2,1 \times 10^{11} \mathrm{~cm}^{-2}$. Considerando os valores dos parâmetros do poço: $\Delta_{1}=162,5 \mathrm{meV}, \Delta_{2}=97,7 \mathrm{meV}, m^{*}=0,075$ e $\epsilon=12,6$, conjuntamente com o valor medido da concentração eletrônica, determinou-se que o poço possui duas sub-bandas ocupadas, mas a segunda delas encontra-se quase vazia.

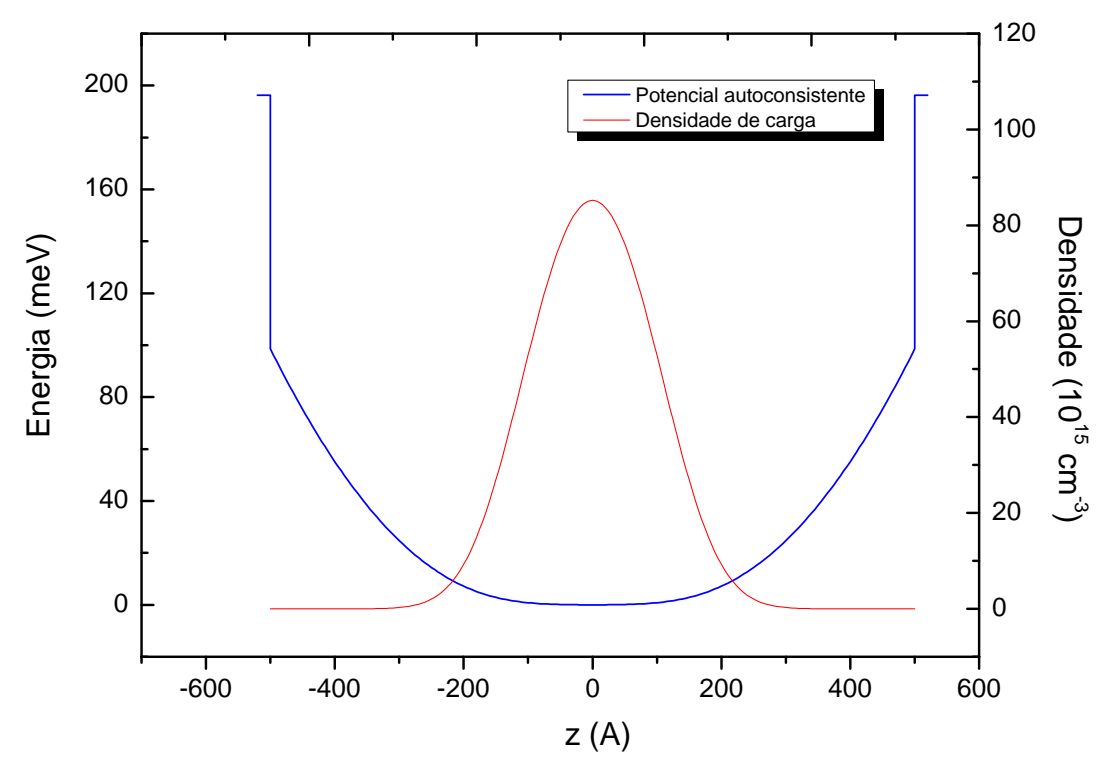

Figura 2.9: Potencial autoconsistente total e a densidade de carga total para um poço de $W=$ $1000 \AA$ e $n_{s}=2,1 \times 10^{11} \mathrm{~cm}^{-2}$. 
A Fig.(2.9) mostra o perfil de potencial autoconsistente após convergido, juntamente com a distribuição de densidade de carga eletrônica total. Observa-se que para o valor da concentração eletrônica $n_{s}=2,1 \times 10^{11} \mathrm{~cm}^{-2}$, a ocupação fracionária é $f=0,23$ e a largura efetiva da distribuição de carga no poço é $W_{e}=230 \AA$. A Tabela (2.4) mostra os dois níveis de energia obtidos através do nosso programa, e também se mostra os valores obtidos experimentalmente. Experimentalmente não foi possível encontrar a concentração da segunda sub-banda pelo fato dela estar quase vazia.

\begin{tabular}{cccc}
\hline \hline Sub-banda & Energia $(\mathrm{meV})$ & $\begin{array}{c}n_{s}^{(i)} \\
\left(10^{11} \mathrm{~cm}^{-2}\right) \\
\text { teórico }\end{array}$ & $\begin{array}{c}n_{s}^{(i)}\left(10^{11} \mathrm{~cm}^{-2}\right) \\
\text { experimental }\end{array}$ \\
\hline \hline 1 & 2,55 & 2,01 & 2,00 \\
2 & 8,68 & 0,09 & - \\
\hline \hline
\end{tabular}

Tabela 2.4: Energias e densidades de carga para um poço parabólico com parâmetros: $W=1000$ $\AA, n_{s}=2,1 \times 10^{11} \mathrm{~cm}^{-2}, \Delta_{1}=162,5 \mathrm{meV}$ e $\Delta_{2}=97,7 \mathrm{meV}$. Neste poço duas sub-bandas estão ocupadas, mas a segunda se encontra quase vazia.

Na Fig.(2.10) mostra-se a distribuição de densidade de carga para cada uma das subbandas e também a densidade total para o poço de largura $W=1000 \AA$ e densidade superficial de carga $n_{s}=2,1 \times 10^{11} \mathrm{~cm}^{-2}$.

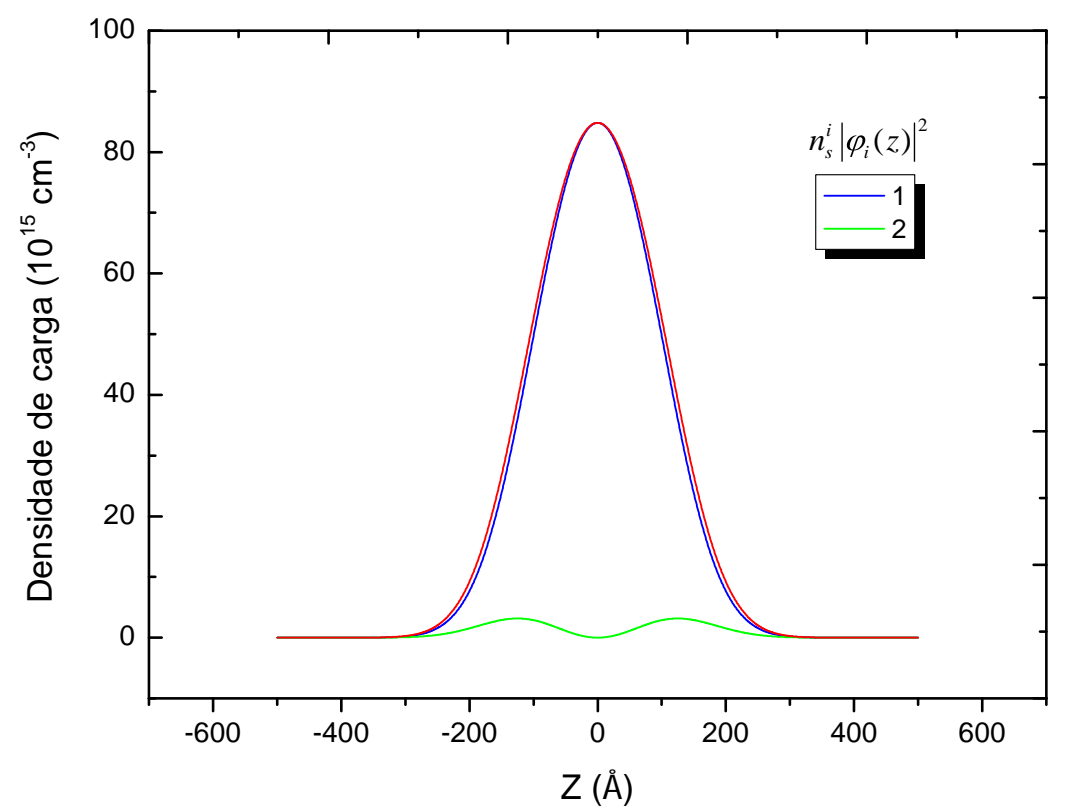

Figura 2.10: Distribuição da densidade de carga para cada uma das sub-bandas e a densidade de carga total para um poço de $W=1000 \AA$ e $n_{s}=2,1 \times 10^{11} \mathrm{~cm}^{-2}$. 
- Amostra A2580: $W=1000 \AA$ e $n_{s}=3,2 \times 10^{11} \mathrm{~cm}^{-2}$.

Vamos mostrar os resultados dos cálculos autoconsistentes para a determinação da estrutura de sub-bandas dessa amostra. Também são comparados estes valores teóricos com os obtidos experimentalmente.

Para determinar autoconsistentemente os autovalores de energia, perfil de potencial e distribuição de cargas desse poço de largura $W=1000 \AA$, utilizou-se a densidade superficial de carga obtida das medidas de magnetotransporte $n_{s}=3,2 \times 10^{11} \mathrm{~cm}^{-2}$, levando em conta os parâmetros do poço: $\Delta_{1}=162,5 \mathrm{meV}, \Delta_{2}=97,7 \mathrm{meV}, m^{*}=0,075 \mathrm{e}$ $\epsilon=12,6$. Determinou-se que para a concentração de portadores dada, o poço possui duas sub-bandas ocupadas.

A Tabela (2.5) apresenta os autovalores de energia obtidos através dos cálculos autoconsistentes, juntamente com as densidades de carga em cada sub-banda. Também mostra-se os valores obtidos experimentalmente. A Fig.(2.11) mostra a distribuição da densidade de carga eletrônica total e também o perfil de potencial autoconsistente, após atingida a convergência. Observa-se que para a concentração de portadores $n_{s}=3,2 \times 10^{11}$ $\mathrm{cm}^{-2}$, a ocupação fracionária no poço é $f=0,35$ e a largura efetiva da distribuição de carga no poço é $W_{e}=350 \AA$.

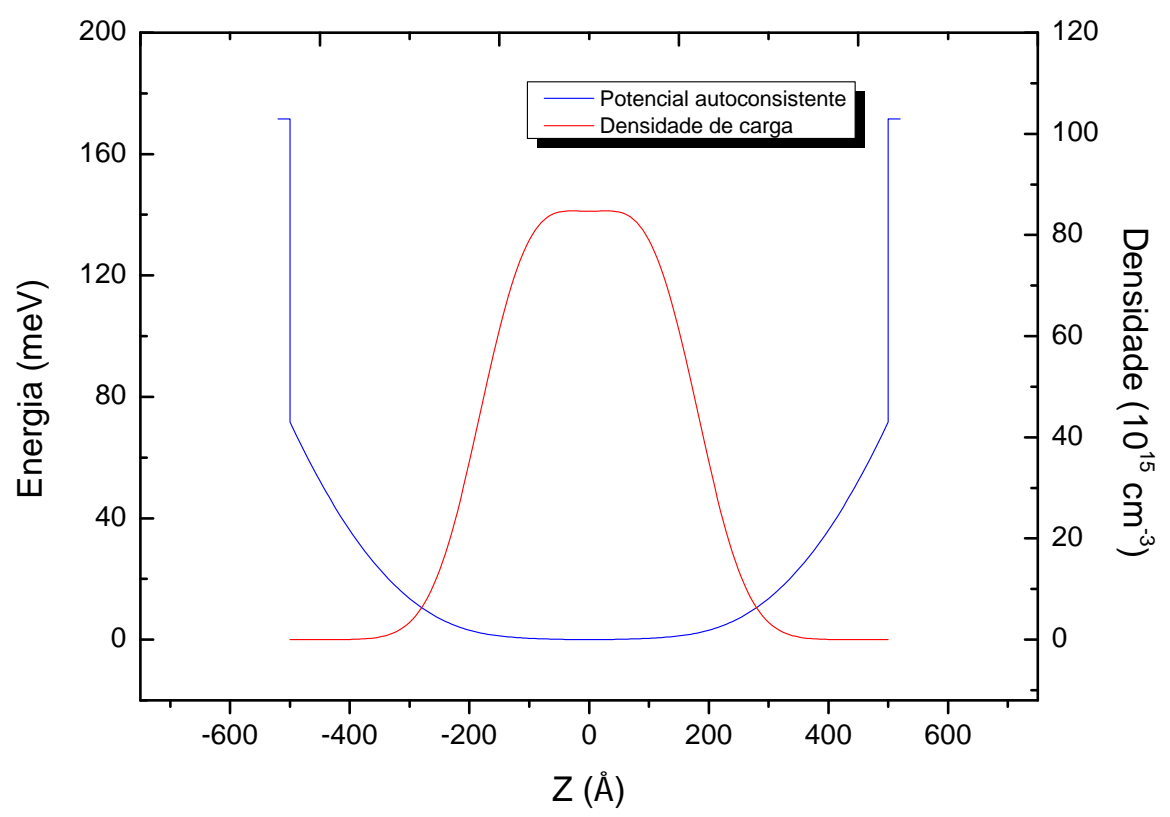

Figura 2.11: Potencial autoconsistente total convergido e a distribuição de densidade de carga total para um poço de $W=1000 \AA$ e $n_{s}=3,2 \times 10^{11} \mathrm{~cm}^{-2}$. 


\begin{tabular}{cccc}
\hline \hline Sub-banda & Energia $(\mathrm{meV})$ & $\begin{array}{c}n_{s}^{(i)}\left(10^{11} \mathrm{~cm}^{-2}\right) \\
\text { teórico }\end{array}$ & $\begin{array}{c}n_{s}^{(i)}\left(10^{11} \mathrm{~cm}^{-2}\right) \\
\text { experimental }\end{array}$ \\
\hline \hline 1 & 1,91 & 2,32 & 2,45 \\
2 & 6,49 & 0,88 & 0,82 \\
\hline \hline
\end{tabular}

Tabela 2.5: Energias e densidades de carga para um poço parabólico com parâmetros: $W=1000$ $\AA, n_{s}=3,2 \times 10^{11} \mathrm{~cm}^{-2}, \Delta_{1}=162,5 \mathrm{meV}$ e $\Delta_{2}=97,7 \mathrm{meV}$. Neste poço duas sub-bandas estão ocupadas.

Na Fig.(2.12) mostra-se a distribuição de carga para cada uma das sub-bandas e também a distribuição da densidade total.

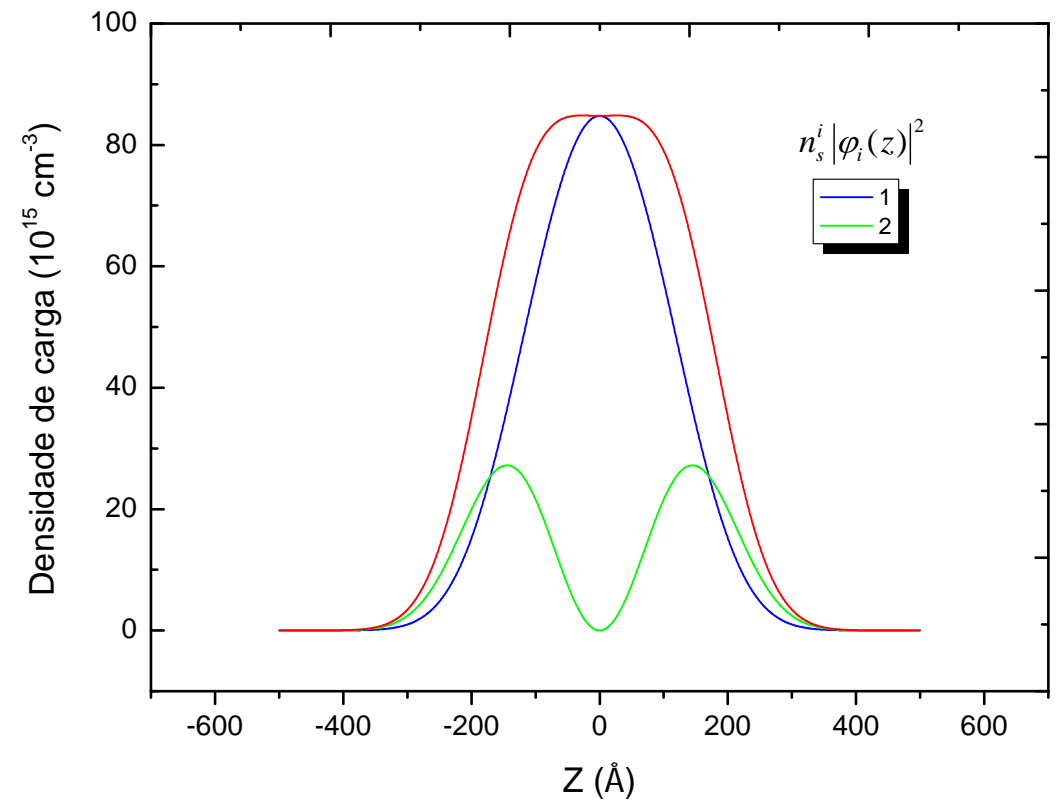

Figura 2.12: Distribuição da densidade de carga para cada uma das sub-bandas e a densidade de carga total para um poço de $W=1000 \AA$ e $n_{s}=3,2 \times 10^{11} \mathrm{~cm}^{-2}$. 


\section{Capítulo 3}

\section{Crescimento, Processamento e Caracterização das Amostras}

\subsection{Crescimento por MBE}

As amostras estudadas neste trabalho, contendo poços quânticos parabólicos, foram crescidos pela técnica de epitaxia por feixe molecular (MBE, Molecular Beam Epitaxy) [72]. Estas amostras foram crescidas no nosso grupo de pesquisa pelo Prof. Dr. Alain A. Quivy, no Laboratório de Novos Materiais Semicondutores (LNMS) do Instituto de Física da Universidade de São Paulo (IFUSP).

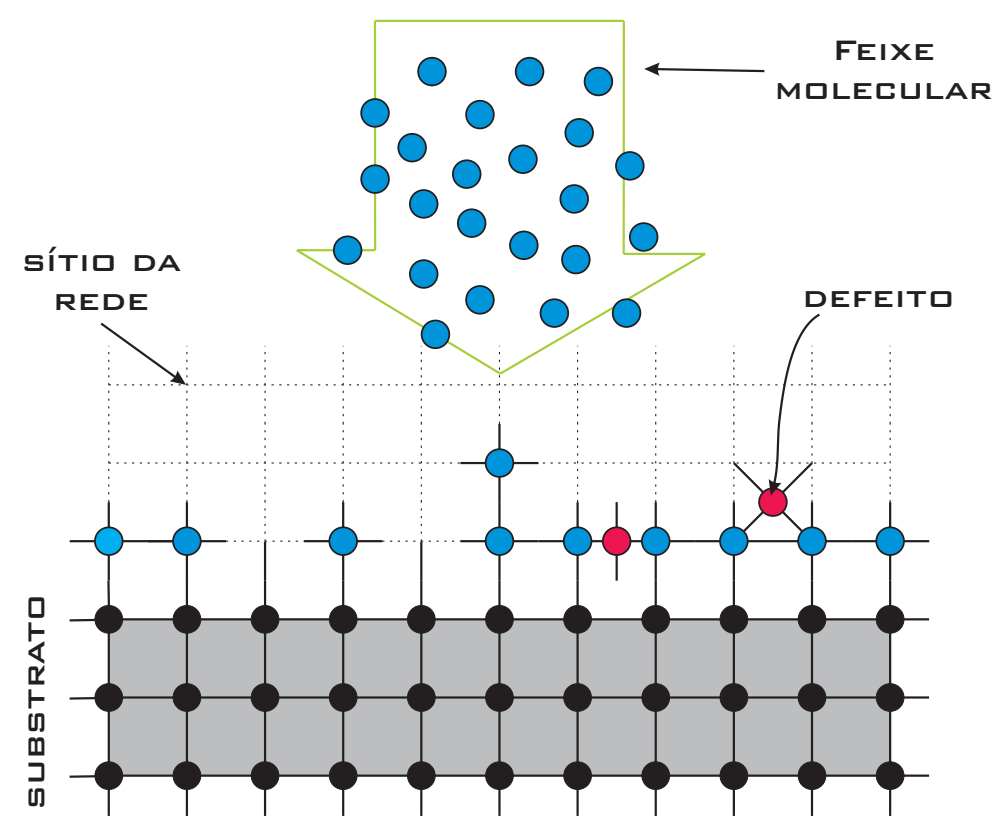

Figura 3.1: Representação esquemática do crescimento de amostras através da técnica de Epitaxia por feixe molecular (MBE). 
O princípio dessa técnica baseia-se na evaporação térmica de elementos ultra puros para gerar um feixe de átomos ou moléculas, o qual incide sobre um substrato cristalino aquecido. Ao incidir os átomos ou as moléculas sobre o substrato, eles tendem a se posicionar nos sítios da rede, e assim, forma-se uma camada fina estruturalmente idêntica à do material sobre o qual foi depositado. Dessa forma, o crescimento tende a seguir a estrutura cristalina do substrato.

Na Fig.(3.1) mostra-se como o feixe de átomos ou moléculas tendem a se posicionar nos sítios da rede. Algumas situações como aquelas representadas pelos círculos vermelhos são possíveis, embora menos frequentes.

O equipamento é composto por tres câmaras de ultra vácuo isoladas entre se por válvulas do tipo gaveta. A primeira câmara é a câmara de entrada e saída das amostras. Na segunda câmara é realizado um processo de degaseificação dos substratos antes de iniciar-se o crescimento. O processo de crescimento ocorre na terceira câmara, onde estão localizadas as células de efusão. Nessas células são aquecidos os elementos ultra puros até começarem a evaporar-se, e o feixe molecular, produto da evaporação, é colimado e direcionado para o substrato, onde os elementos reagem e condensam-se. Para as nossas amostras, as células de efusão contém os siguintes materiais sólidos: gálio $(G a)$, arsênio $(A s)$, alumínio $(A l)$, e silício $(S i)$, todos com alto grau de pureza. Entre o substrato e cada célula existe um obturador pneumático que permite interromper o fluxo dos feixes de maneira quase imediata. A quantidade de átomos ou moléculas que incide sobre o substrato é diretamente proporcional ao tempo de abertura do obturador.

Nesta câmara também encontra-se uma fonte para o aquecimento do substrato e um motor que o faz girar de forma a garantir a uniformidade dos filmes durante o crescimento. O sistema também tem um conjunto de camisas de nitrogênio líquido que atuam como dissipadores térmicos evitando que a temperatura das células de efusão influencie as vizinhas ao mesmo tempo que mantém a temperatura de crescimento do substrato constante.

O canhão de elétrons e a tela fluorescente são necessários para monitorar o crescimento através de uma técnica chamada RHEED (reflection high-energy electron diffraction). Consiste basicamente em monitorar a projeção dos elétrons emitidos pelo canhão que, após serem difratados na superfície da amostra, são projetados na tela fosforescente gerando um padrão de difração que permite obter informações sobre a estrutura cristalina das camadas crescidas. Permite também calibrar o fluxo dos feixes e controlar a composição da liga e o número de camadas depositadas [73]. A Fig.(3.2) mostra o esquema de um sistema típico de MBE.

As condições e o sistema descritos acima, possibilitam produzir filmes com alta qualidade cristalina, imprescindível para a produção de diversas estruturas semicondutoras. 


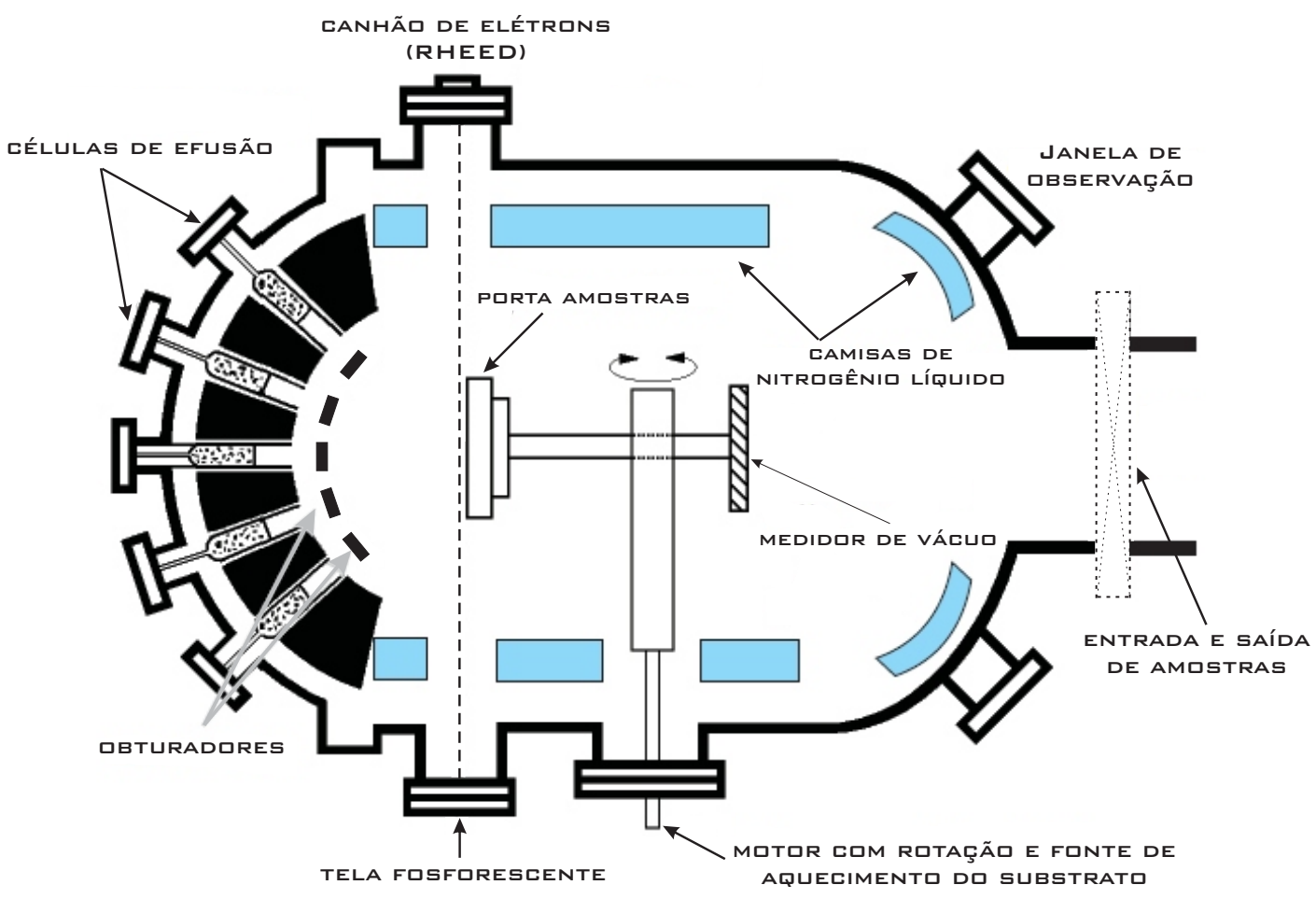

Figura 3.2: Esquema genérico de um sistema $M B E$ onde são crescidas as heteroestruturas semicondutoras.

\subsection{Arquitetura dos poços quânticos parabólicos}

As heteroestruturas semicondutoras contendo os poços quânticos foram crescidas sobre substratos semi-isolantes de GaAs com orientação cristalográfica (100) por epitaxia de feixe molecular. O esquema de crescimento das amostras seguiu as seguintes etapas: Primeiramente é crescida uma camada buffer de $1000 \AA$ de GaAs sobre o substrato, para a obtenção de uma superficie de alta qualidade cristalina, seguida de uma super-rede de 20 períodos de $A l A s / G a A s$, para a contenção das impurezas do substrato, e de outra camada de $8000 \AA ̊$ de $G a A s$. A seguir, com a concentração de $A l$ variando linearmente de $0 \%$ até $31 \%$ cresce-se uma rampa de $500 \AA$ de espessura, seguida de uma camada de $A l G a A s$ com a concentração uniforme de $31 \%$ de $A l$. Esta camada com a concentração constante de $31 \%$ constitui a barreira interna do poço e contém uma camada de dopagem planar de silício $\left(\delta_{S i}\right)$. Após a camada $\delta_{S i}$, existe uma outra camada de AlGaAs chamada de camada espaçadora ou spacer também com concentração $31 \%$ de $A l$. A finalidade desta camada é separar o gás de elétrons confinado no poço dos centros espalhadores coulombianos localizados na camada $\delta_{S i}$. A seguir é crescido o poço quântico parabólico com parâmetros de acordo com o desejado, seguido de uma outra camada de espaçamento e uma outra camada planar de dopantes de $S i$. Finalmente é crescida uma camada de proteção de $500 \AA$ de $A l G a A s$ com dopagem planar de silício no meio, com a finalidade de neutralizar 
as cargas da superfície da amostra.

Na Fig.(3.3) mostra-se a composição das camadas que compõem as amostras de poços quânticos parabólicos, juntamente com o perfil da concentração de alumínio na liga de AlGaAs.

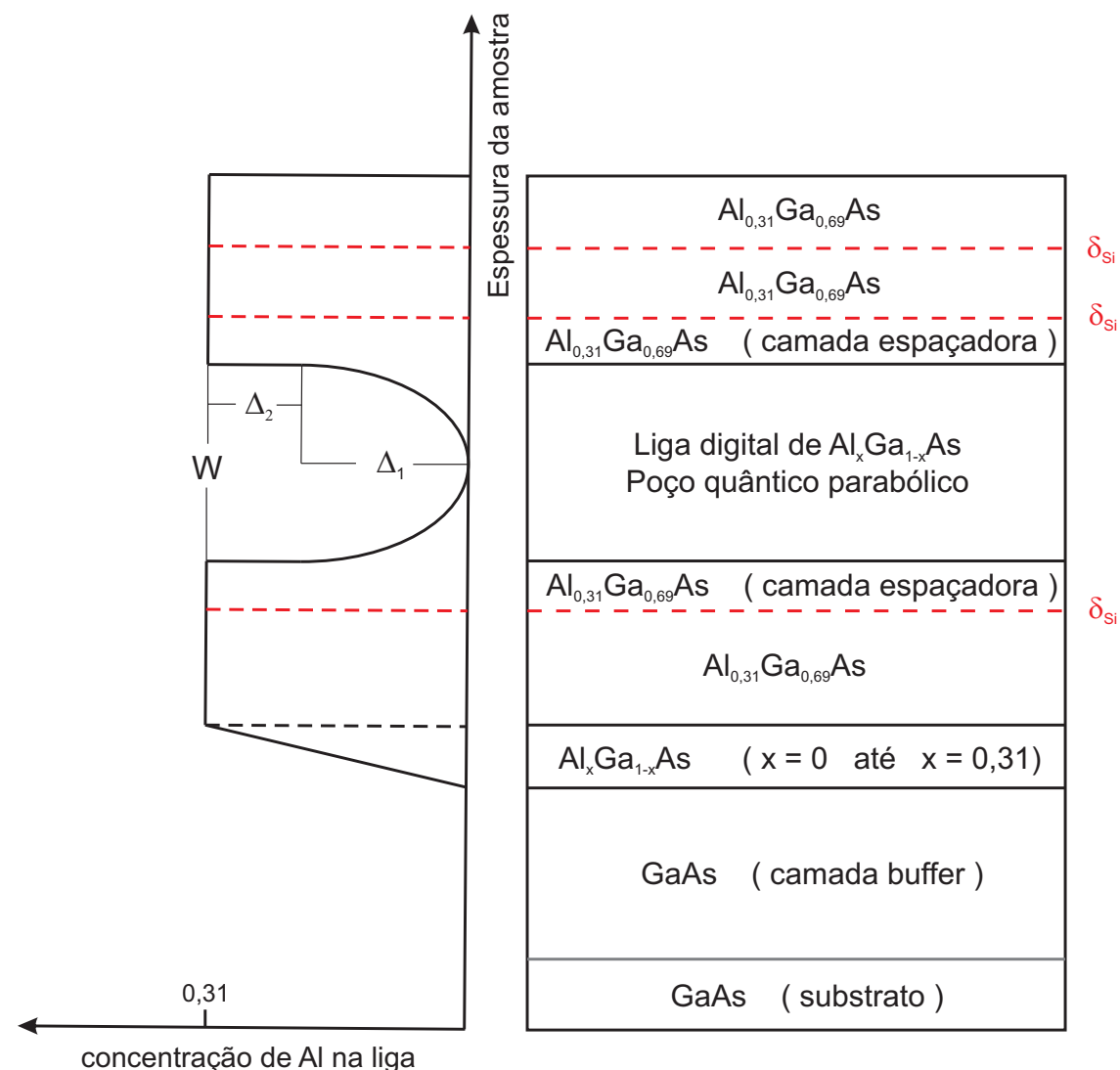

Figura 3.3: Representação esquemática da sequencia das camadas semicondutoras que compõem as amostras de poços parabólicos, assim como a concentração de alumínio na liga.

O crescimento do poço quântico parabólico é feito com uma estrutura denominada liga digital, em contraposição à denominada liga analógica. Isso é feito com a finalidade de se obter amostras com mobilidades melhores, pois amostras de poços quânticos crescidas com a técnica de liga digital apresentam melhores resultados em medidas ópticas e elétricas $[74,75]$.

O crescimento de uma liga analógica de $A l_{x} G a_{1-x} A s$ consiste na deposição dos tres elementos simultaneamente nas proporções desejadas, enquanto o equivalente digital consiste na deposição de camadas finas de GaAs e $A l A s$ em períodos de espessura $L_{p}$, sendo que o porcentual da quantidade do número de camadas de cada material definirá a composição média da liga de $A l G a A s$. Por exemplo, se quisermos ter uma liga de $A l G a A s$ 
com $30 \%$ de alumínio, podem ser crescidas 3 camadas de AlAs e 7 camadas de GaAs em um período $L_{p}$. Na média o conjunto de todos estes períodos com diferentes composições na liga produzirão o potencial parabólico. O valor mais adequado para $L_{p}$ é de $20 \AA$, uma vez que esta largura permite o tunelamento de elétrons [76].

A razão de crescer as amostras com a técnica da liga digital é a mobilidade das cargas. Em sistemas crescidos através da técnica da liga analógica, o espalhamento por desordem na liga se traduz numa baixa mobilidade dos portadores. Cálculos teóricos tem mostrado que o espalhamento por desordem é quem limita a mobilidade dos portadores em poços crescidos pela técnica analógica. Entretanto, nos poços quânticos de liga digital este espalhamento é minimizado e os portadores tem maiores mobilidades [77, 78].

\subsection{Preparo das amostras para a realização de medi- das}

As medidas de transporte em heteroestruturas semicondutoras exige uma preparação prévia das amostras. Antes de fazer as medidas de magnetotransporte é necessário primeiramente gravar uma barra Hall na amostra. Após a gravação da barra Hall é feito a difusão dos contatos ôhmicos. No caso das amostras com porta metálica ou gate, é feito um processo de litografia eletrônica numa camada de polímero PMMA depositada sobre a barra Hall, com a finalidade de criar uma barreira, e depois foi depositada a porta de ouro. Os processos de gravação da barra Hall foram feitos no Instituto de Física de São Carlos (IFSC) da USP, enquanto a evaporação da porta foi feita na Universidade estadual de Campinas (UNICAMP). A seguir, vamos descrever com mais detalhe estes processos.

\subsubsection{Gravação da barra Hall}

A implantação da barra Hall nas amostras foi feita através da técnica de fotolitografia e corrosão química. O desenho da barra Hall é mostrado na Fig.(3.4). A região de interesse consiste apenas na pequena área central (cor amarelo) medindo $200 \mu \mathrm{m} \times 500 \mu \mathrm{m}$, onde possamos assumir que a densidade de corrente seja uniforme.

O processo consiste nas seguintes etapas:

- Primeiramente devemos limpar as amostras com acetona, álcool, água e seca-la com nitrogênio gasoso $\left(N_{2}\right)$.

- Depois, fixamos a amostra (a vácuo) pela base (substrato) em um aparelho chamado spinner, pingamos algumas gotas de um revelador fotossensível (fotorresiste) na superfície da amostra e ela é posta em rotação (rotação do spinner $7000 \mathrm{rpm}$ ). Este 
passo deve ser feito sobre luz amarela para que o fotorresiste não seja sensibilizado. Assím, o fotorresiste fica espalhado uniformemente pela superfície da amostra.

- Em seguida, é feito um pre-aquecimento colocando a amostra num forno a $100^{\circ} \mathrm{C}$ por 20 min. Este processo também deve ser feito sob luz amarela.

- Coloca-se a amostra em uma fotoalinhadora, posiciona-se a máscara de litografia com barra Hall sobre a amostra e expõe-se a amostra à luz ultravioleta por 40s. Deste modo, a superfície do fotorresiste que não estava protegida pela mascara é sensibilizada, sofrendo alterações em sua composição química.

- Agora, mergulhamos a amostra numa solução reveladora por aproximadamente 1 min. Em seguida a amostra é lavada com água deionizada e secada com nitrogênio. A revelação extrai o fotorresiste sensibilizado e assim o ataque químico a seguir não agride a região protegia pelo fotorresiste não sensibilizado.

- Depois é feita a corrosão química, com uma solução de $\mathrm{H}_{3} \mathrm{PO}_{4}: \mathrm{H}_{2} \mathrm{O}_{2}: \mathrm{H}_{2} \mathrm{O}$ na proporção de 1:8:80. O tempo do ataque químico depende da amostra, neste caso foi de $70 \mathrm{~s}$.

- Finalmente, retiramos o fotorresiste com acetona, e limpamos os resíduos com isopropanol. Assim, a barra Hall está instalada e a amostra está pronta para colocar os contatos ôhmicos.

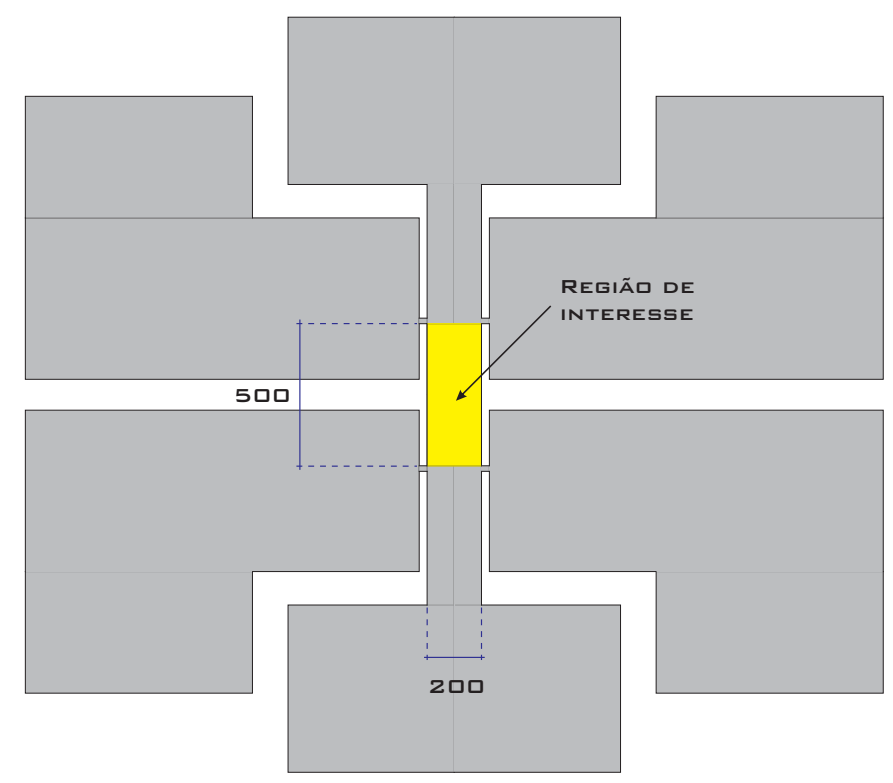

Figura 3.4: Desenho da barra Hall litografada nas amostras. Mostra-se a região de interesse com largura de $200 \mu \mathrm{m}$ e comprimento de $500 \mu \mathrm{m}$. 


\subsubsection{Difusão dos contatos ôhmicos}

Um contato ôhmico é basicamente uma junção metal-semicondutor na qual a relação entre a corrente elétrica e a tensão através da amostra é linear. Esses contatos são utilizados para aplicar corrente elétrica, assim como para medir as diferenças de potenciais.

Para fazer os contatos ôhmicos, usamos o método de difusão, que consiste simplesmente em colocar um pouco de índio nas bordas da barra Hall e em seguida aquece-lo a $400{ }^{\circ} \mathrm{C}$ durante 4 minutos numa atmosfera de nitrogênio. Nessas circunstancias o índio difunde verticalmente e forma um canal metálico ao longo de toda a heteroestrutura, o que nos dá acesso à porção da heteroestrutura que possui a estrutura de interesse.

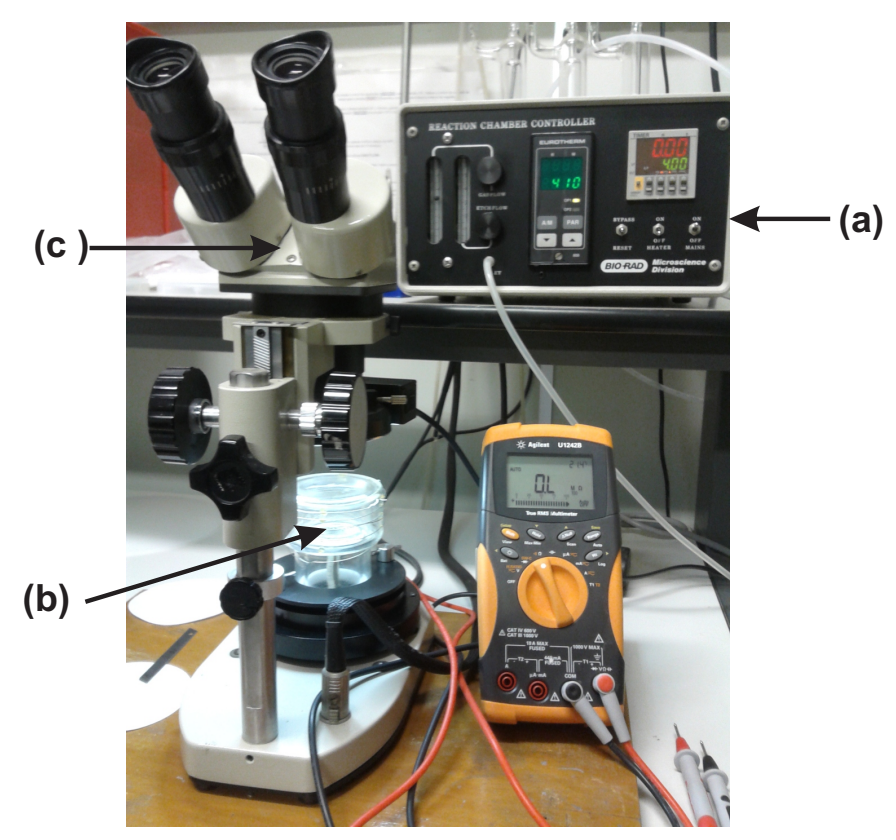

Figura 3.5: Equipamento para fazer a difusão dos contatos ôhmicos na amostra com barra Hall. (a) Módulo eletrônico que controla a temperatura da câmara da amostra. (b) Câmara da amostra, contem um sistema de aquecimento e é mantida numa atmosfera de $N_{2}$ gasoso. (c) Microscópio para colocar os contatos de In.

\subsubsection{Evaporação da porta metálica com barreira}

O gate ou porta, é um contato não ôhmico muito utilizado em transistores de efeito de campo (FET, field effect transistor), cuja função é controlar a concentração de portadores 
através da diferença de potencial aplicado a ele, o que cria uma região de depleção nos arredores da porta metálica. Basicamente trabalha como um capacitor, onde uma das placas é o contato metálico e a outra o gás de elétrons [79]. A diferença dos contatos ôhmicos, neste tipo de contato não há fluxo de cargas entre a porta e o gás bidimensional de elétrons, o que é conhecido como corrente de fuga.

Em primeiro lugar, é depositado um filme de um polímero de PMMA (polimetilmetacrilato) com espessura aproximada de $0,3 \mu \mathrm{m}$, que recobre a superfície da amostra. Esta deposição é feita exatamente como depositamos o fotorresiste para a litografia da barra Hall. A seguir, a barreira no PMMA é feita por litografia eletrônica, ou seja, é desenhada com um feixe de elétrons. No nosso caso a barreira é perpendicular ao canal de corrente da barra Hall, e possui uma largura de aproximadamente 350nm. O processo é feito num microscópio eletrônico de varredura (SEM, scanning electron microscope), onde o feixe de elétrons sensibiliza a região de PMMA exposta. Depois de exposto ao feixe de elétrons, efetuamos a revelação, processo químico no qual as regiões no PMMA sensibilizadas pelo feixe são removidas, assim, o PMMA fica cobrindo toda a superfície da amostra, exceto na região da barreira. Na Fig.(3.6) mostra-se a vista de perfil do canal de corrente da barra Hall com o esquema geral de camadas na amostra e com as camadas de PMMA e ouro depositadas na superfície da amostra.

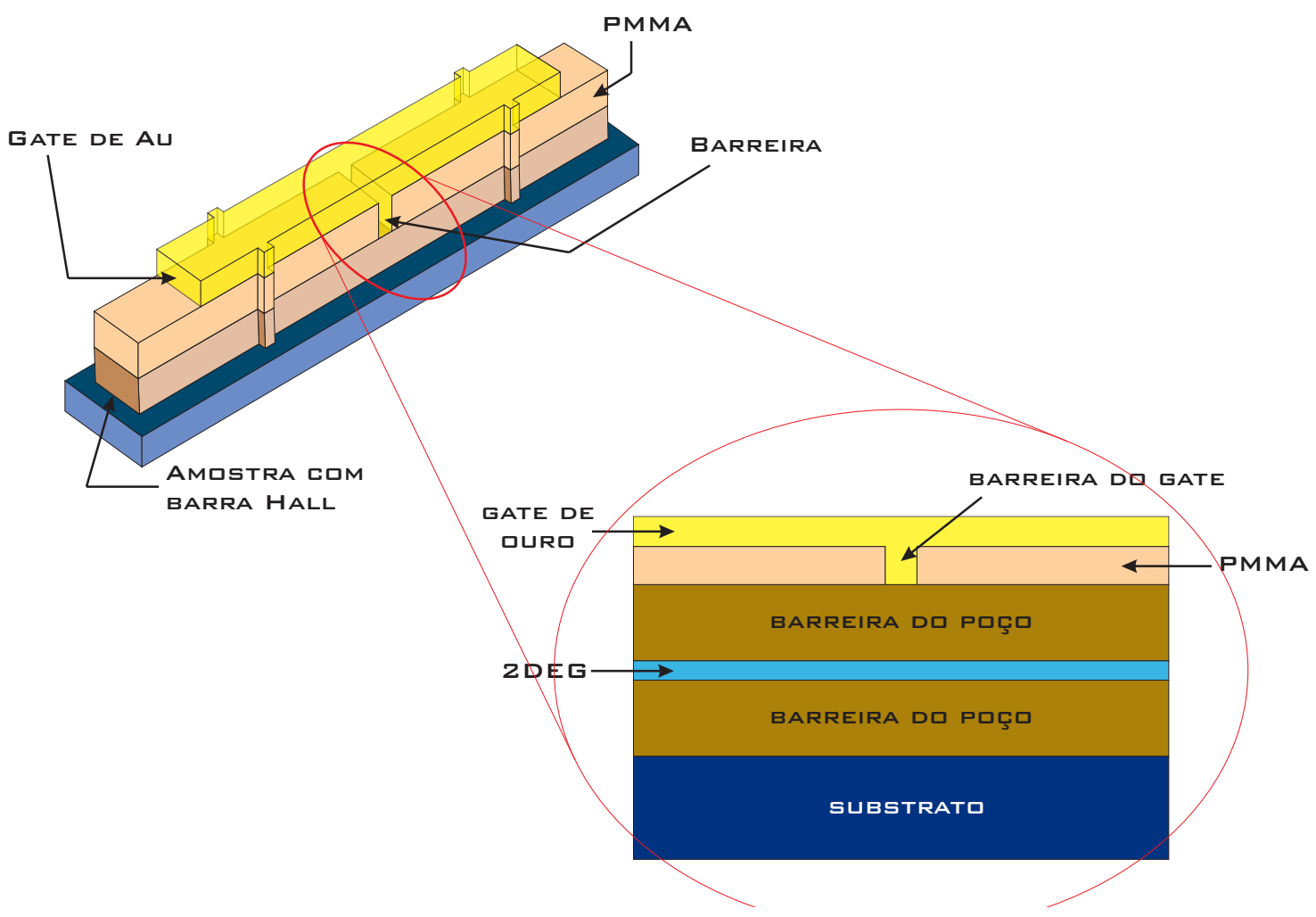

Figura 3.6: Mostra-se o esquema da deposição de camadas de PMMA sobre a barra Hall com a barreira feita por feixe de elétrons, e a camada de ouro (porta). 
Enquanto ao procedimento de fabricação, consiste basicamente na evaporação e subsequente deposição do metal, nesse caso ouro e titânio, sobre a superfície da amostra. Usa-se um pequeno pedaço de acetato com um furo central de aproximadamente $1 \mathrm{~mm}$ de diâmetro, e colocamos-lo sobre a amostra, tomando cuidado de posicionar o furo sobre a região de interesse, como se mostra na Fig.(3.7b). Depois a amostra é colocada numa câmara de vácuo de uma evaporadora, dentro da qual encontra-se um cadinho metálico que contém o metal a ser evaporado. A Fig.(3.7a) mostra o esquema da câmara de evaporação.

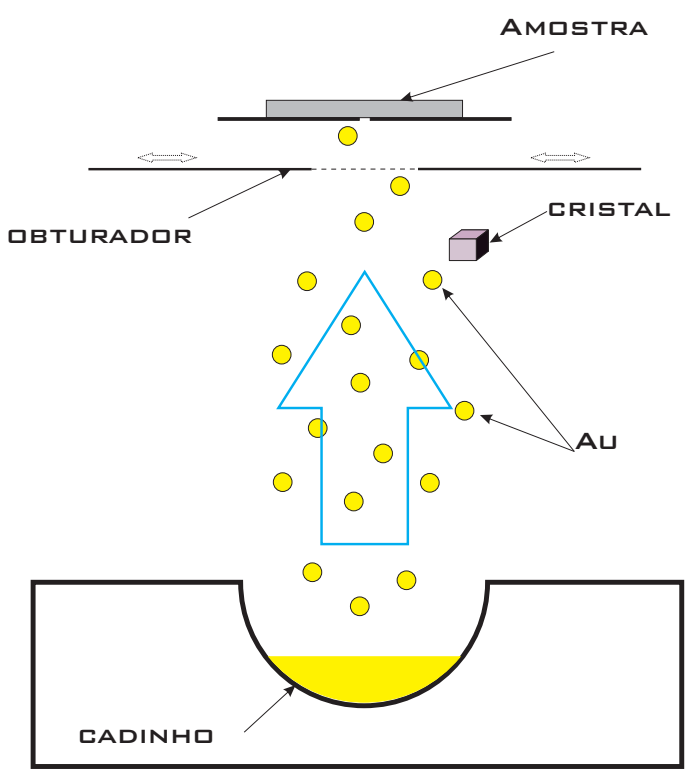

(a)

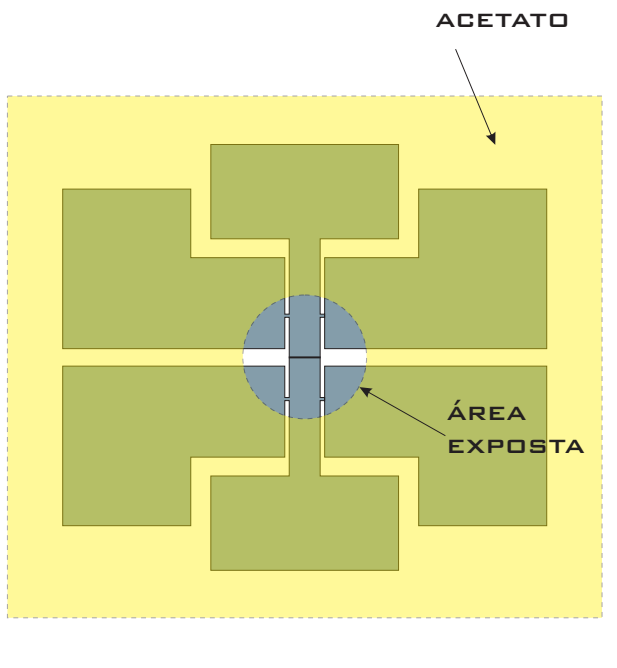

(b)

Figura 3.7: (a) Câmara de evaporação do ouro e titânio. (b) Região da amostra abaixo do acetato que fica exposta ao fluxo de ouro/titânio.

O metal é aquecido por efeito Joule ao aplicarnos uma corrente elétrica constante através do cadinho, e o fluxo de evaporação é medido através da oscilação de um cristal próximo da amostra. Conhecendo a taxa de deposição do ouro/titânio no cristal (da ordem de $\AA$ /s e supostamente igual aquela sobre a amostra), podemos depositar o filme metálico com precisão de um ou dois nanometros. Existe um problema quando só é depositado ouro $(A u)$, pois ocorre que ele difunde no GaAs mesmo a temperatura ambiente, desse modo, após algum tempo ele atinge o gás de elétrons, destruindo a amostra. Por conta disso, primeiro é depositado um filme de titânio, que funciona como barreira de difusão, antes de fazer a deposição do ouro. Depois colocamos com prata ou soldamos com índio um fio de ouro na porta, através do qual conectamos a amostra aos instrumentos de medida. 
Finalmente, depois de fazer todos esses procedimentos na amostra, desde a gravação da barra Hall até a doposição da porta metálica, a amostra fica pronta para fazer a caracterização por meio de transporte. A Fig.(3.8) mostra o esquema da amostra pronta para as medidas de transporte.

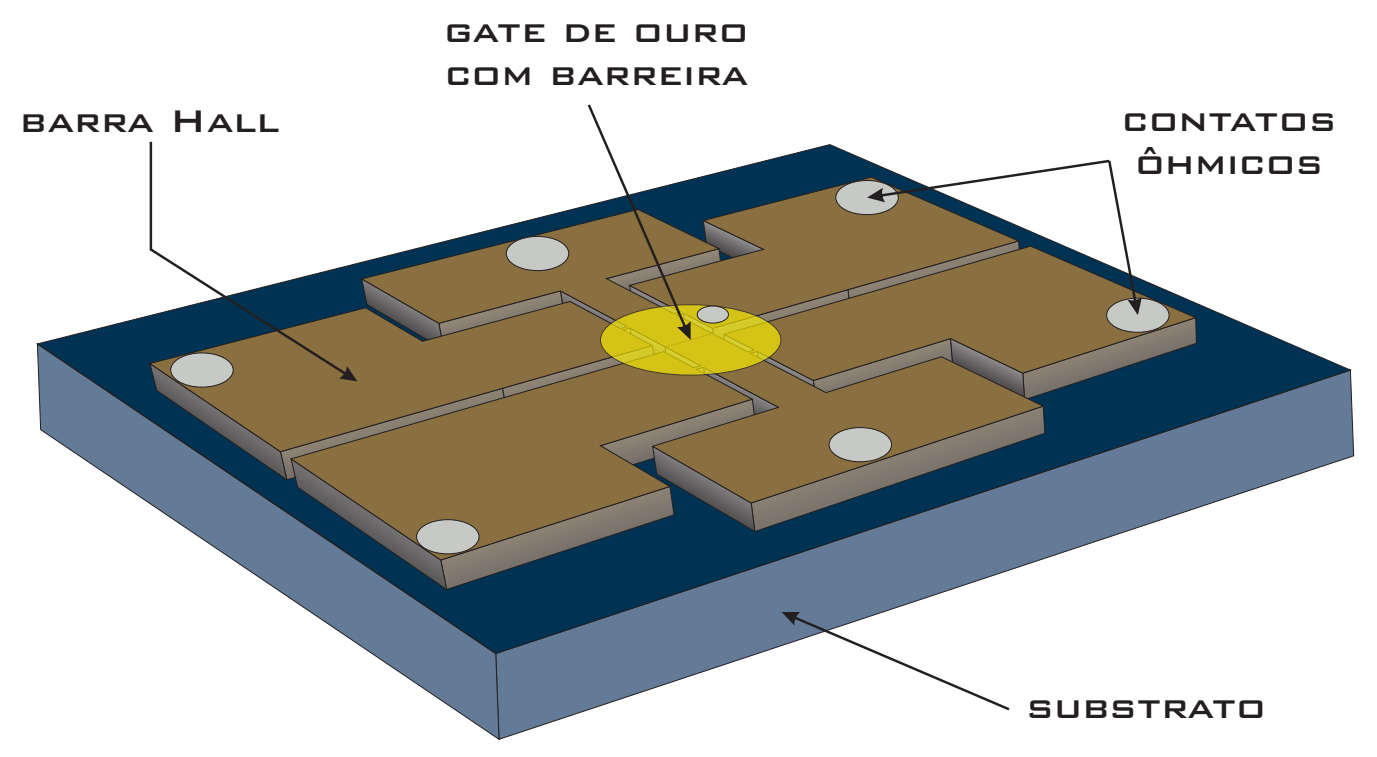

Figura 3.8: Esquema de uma amostra com porta ou gate pronta para as medidas de transporte.

\subsection{Caracterização por meio de transporte}

Todos os resultados apresentados aqui, tem como base medidas de magnetorresistência $\left(R_{x x}\right)$ e resistência Hall $\left(R_{x y}\right)$ a temperaturas do Hélio líquido ${ }^{4} H e(4,2 \mathrm{~K})$ e do Hélio superfluido $(1,5 \mathrm{~K})$. As medidas foram realizadas com campo magnético perpendicular e paralelo à superficie das amostras, utilizando as facilidades experimentais do Laboratório de novos materiais semicondutores (LNMS) do Instituto de Física da Universidade de São Paulo.

\subsubsection{Equipamentos utilizados}

O equipamento que possui o LNMS, consiste de uma bobina supercondutora que pode atingir campos até de $15 \mathrm{~T}$ e de um criostato com sistema VTI (Variable Temperature Insert) da Oxford Instruments que permite o controle da temperatura de 1,5K a 300K. Para o estudo do transporte, as temperaturas baixas são um fator muito importante, nessas 
temperaturas é possível a observação de efeitos quânticos.

No LNMS temos um criostato com uma bobina supercondutora que permanece imersa num banho de ${ }^{4} \mathrm{He}$. A câmara contendo o ${ }^{4} \mathrm{He}$ líquido é isolada através de uma câmara de vácuo, outra câmera mais externa contendo $N_{2}$ líquido permite limitar a diminuição da temperatura até $77 \mathrm{~K}$, e finalmente uma câmara externa em alto vácuo para melhorar o isolamento térmico do sistema.

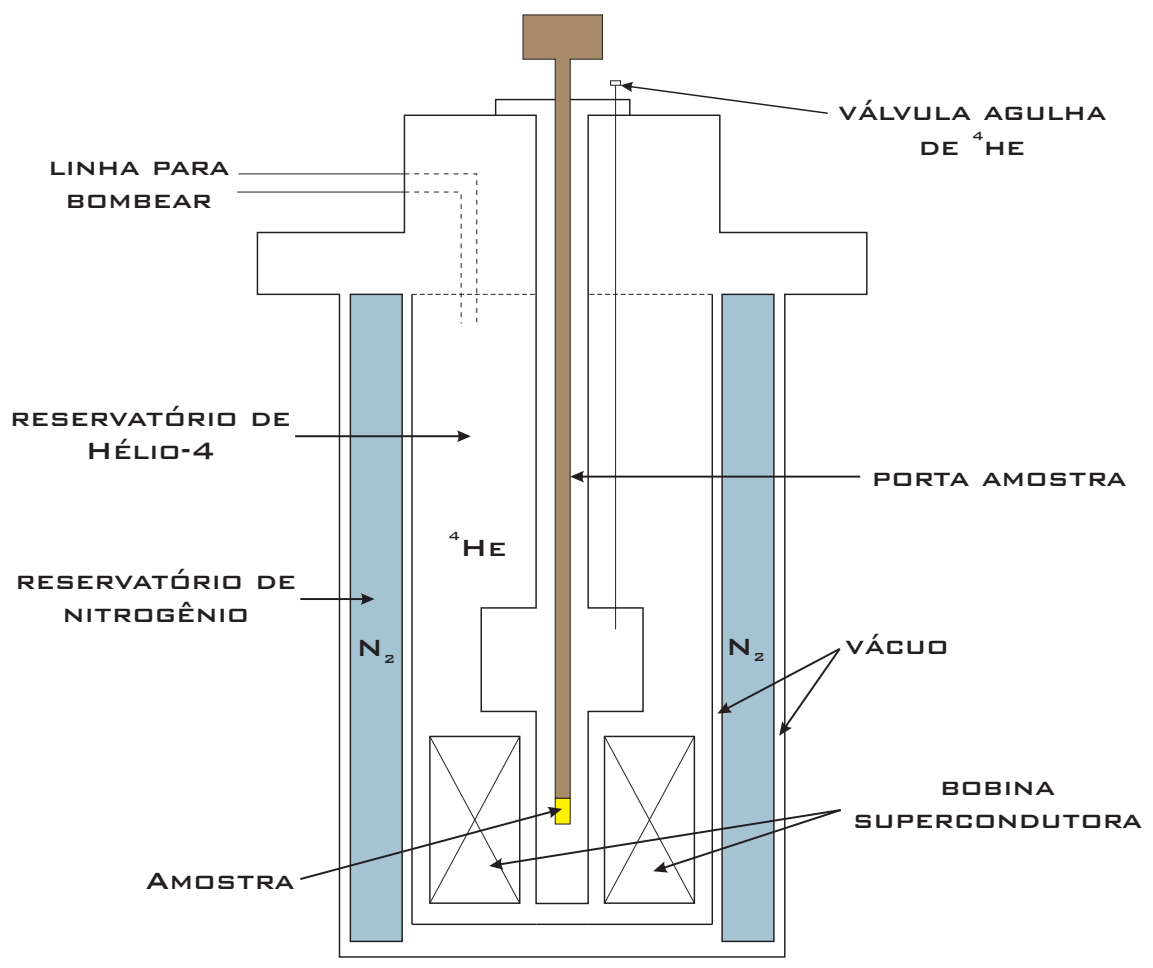

Figura 3.9: Esquema interno básico do criostato com bobina supercondutora para medidas de transporte no LNMS.

A Fig.(3.9) mostra o esquema básico do nosso sistema VTI. O principio de utilização deste sistema está baseado nas propriedades da temperatura e da pressão do ${ }^{4} \mathrm{He}$. A temperatura do ${ }^{4} \mathrm{He}$ líquido a pressão atmosférica é de $4,2 \mathrm{~K}$, mas diminuindo a pressão do ${ }^{4} \mathrm{He}$ na sua respectiva câmara, a sua temperatura também diminui, assim é possível atingir temperaturas até de 1,5K usando um sistema de bombeamento. A amostra é colocada num porta-amostra, que ao mesmo tempo está imerso diretamente num banho de ${ }^{4} \mathrm{He}$. Este banho de ${ }^{4} \mathrm{He}$ é conectado ao banho de ${ }^{4} \mathrm{He}$ principal através de uma válvula agulha, a qual é usada para injetar ${ }^{4} H e$ na câmara onde está o porta-amostra. A adequada injeção de ${ }^{4} \mathrm{He}$ na câmara da amostra permite estabilizar a temperatura.

Entre os outros equipamentos de medida temos: 
- Um amplificador lock-in modelo EGG 5210 Princeton Applied Research. Ele recebe os sinais de tensão provenientes dos contatos da voltagem longitudinal e transversal, e continua o processo de amplificação e rejeição de ruídos indesejados. Também é utilizado aplicar uma corrente alternada da ordem de $1 \mathrm{~V}$ às amostras.

- Um multímetro programável modelo Hewlett Packard 34401A. Ele envia o sinal de voltagem amplificado ao computador.

- Uma fonte de corrente programável modelo Oxford IPS120-10, utilizada no sistema magnético supercondutor.

- Um controlador de temperatura programável utilizada no sistema VTI modelo Oxford ITC-503.

- Um controlador de nível de Hélio-4 e de nitrogênio modelo Oxford ILM-211.

- Um computador para a aquisição dos dados. Adicionalmente um programma feito em Labview é o responsável pelo controle automático dos equipamentos.

\subsubsection{Técnicas de caracterização de amostras}

A Fig.(3.10) apresenta a disposição dos contatos longitudinal e transversal em uma barra Hall, responsáveis pelas medidas das voltagems longitudinal e de Hall respectivamente. A aquisição de dados é feita lendo valores de voltagems. Assim, é necessario transformar os valores de voltagem em valores de resistência.

O valor da resistência longitudinal ou magnetorresistência é dada utilizando a lei de Ohm por:

$$
R_{x x}=\frac{V_{x x}}{I}
$$

onde $V_{x x}$ é a voltagem longitudinal em função do campo magnético, e $I$ é a corrente aplicada.

Um valor importante de transporte, utilizado para determinar a mobilidade de transporte, é o da resistividade longitudinal (ou resistencia quadrado $R_{\square}$ ), que é dada por $\rho_{x x}=R_{x x} / f$, onde $f$ é o fator de forma que depende da geometria da barra Hall e é dado pela razão entre o comprimento e a largura do canal de corrente da barra Hall, que no nosso caso é $f=\frac{500 \mu m}{200 \mu m}=2,5$. As unidades da resistência e resistividade longitudinais são as mesmas.

A resistência Hall $R_{x y}$ que é equivalente à resistividade $\rho_{x y}$ é simplesmente dada por:

$$
R_{x y}=\frac{V_{x y}}{I}
$$




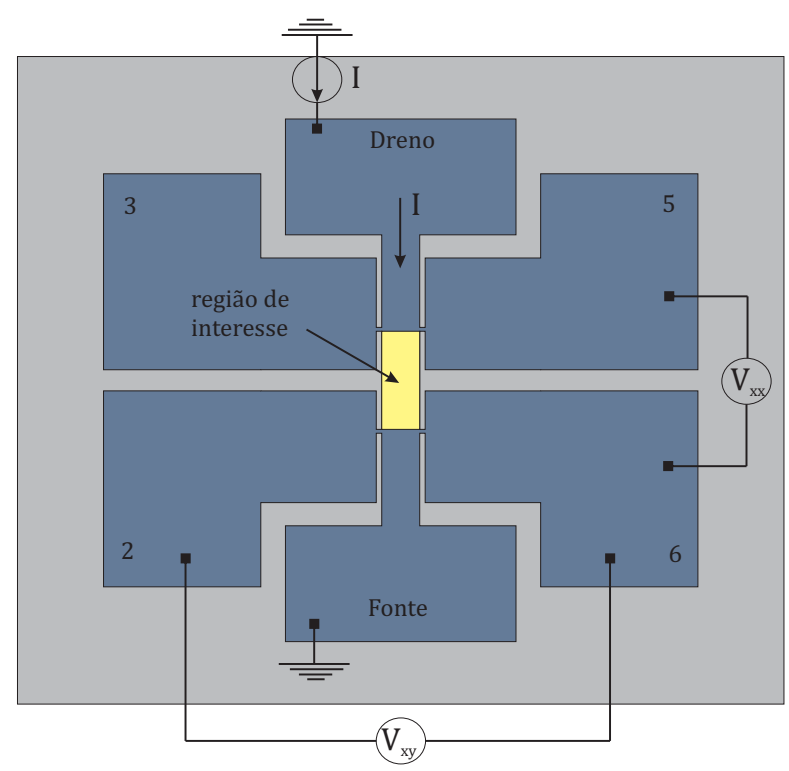

Figura 3.10: Desenho da barra Hall, onde a corrente elétrica é aplicada através dos contatos 1 (fonte) e 4 (dreno). A região de interesse é acessada por os contatos, através dos quais efetuamos as medidas de magnetorresistência e efeito Hall.

onde $V_{x y}$ é a voltagem transversal ou voltagem de Hall em função do campo magnético.

A densidade eletrônica total é obtida na região da curva $R_{x y}(B)$ onde temos um comportamento clássico, ou seja para valores pequenos de campo magnético (geralmente até $0,5 \mathrm{~T}$ ), através da Eq.(1.42) mostrada anteriormente:

$$
n_{s}=\frac{B}{e R_{x y}}
$$

Outro parâmetro importante é a mobilidade de transporte $\mu$, a qual é obtida usando a Eq.(1.43):

$$
\mu=\frac{1}{e \rho_{x x}(0) n_{s}}
$$

onde $\rho_{x x}(0)$ é a resistividade longitudinal a campo magnético zero $(B=0)$. 


\section{Capítulo 4}

\section{Análise e Discussão de Resultados}

Neste capítulo apresentamos a análise dos resultados experimentais das medidas de magnetotransporte e de resistência Hall em heteroestruturas semicondutoras contendo poços quânticos parabólicos (PQW) em configurações de campo magnético perpendicular e paralelo. São apresentadas medidas realizadas em amostras sem porta metálica e em amostras contendo uma porta com barreira.

No caso das medidas de transporte, que compreendem os efeitos Hall e Shubnikovde Haas, as informações que queremos limitam-se às concentrações e mobilidades dos portadores envolvidos no transporte elétrico. Isto porque são essas duas propriedades do gás de elétrons que caracterizam esse transporte de cargas através das amostras.

A implementação do formalismo dos cálculos autoconsistentes descrito no Capítulo 2, permitiu calcular numericamente as concentrações de cada uma das sub-bandas ocupadas nos poços parabólicos. As análises dos resultados das medidas de magnetotransporte também nos fornecem esses valores os quais são comparados com os resultados numéricos.

Realizou-se medidas em uma amostra contendo uma porta metálica de ouro com uma barreira estreita. São feitas medidas em configurações de campo magnético perpendicular e paralelo variando-se a tensão de porta.

As amostras estudadas foram as seguintes:

\begin{tabular}{c|c|c}
\hline \hline Amostra & Largura $(W)$ & \\
\hline \hline A2578 & $W=1000 \AA$ & amostra sem porta \\
A 2580 & $W=1000 \AA$ & amostra sem porta \\
A 2519 & $W=3000 \AA$ & amostra contendo porta com barreira \\
\hline \hline
\end{tabular}

Tabela 4.1: Amostras estudadas no presente trabalho. 


\subsection{Medidas em poços quânticos parabólicos}

Fazendo uso das medidas de resistência longitudinal, resistência de Hall e oscilações Shubnikov-de Haas, determinamos as concentrações e as mobilidades dos elétrons nas amostras A2578 e A2580. Estas medidas de magnetotransporte foram feitas na geometria de barra Hall com campo magnético perpendicular à superfície da amostra e a temperatura de Hélio liquido superfluido $(1,5 \mathrm{~K})$.

\subsubsection{Resultados para a amostra A2578}

A amostra A2578, apresentada no capítulo 2, contem um poço quântico parabólico de largura $W=1000 \AA$. Na Fig.(4.1) mostra-se os resultados para as resistências $R_{x x}$ e $R_{x y}$.

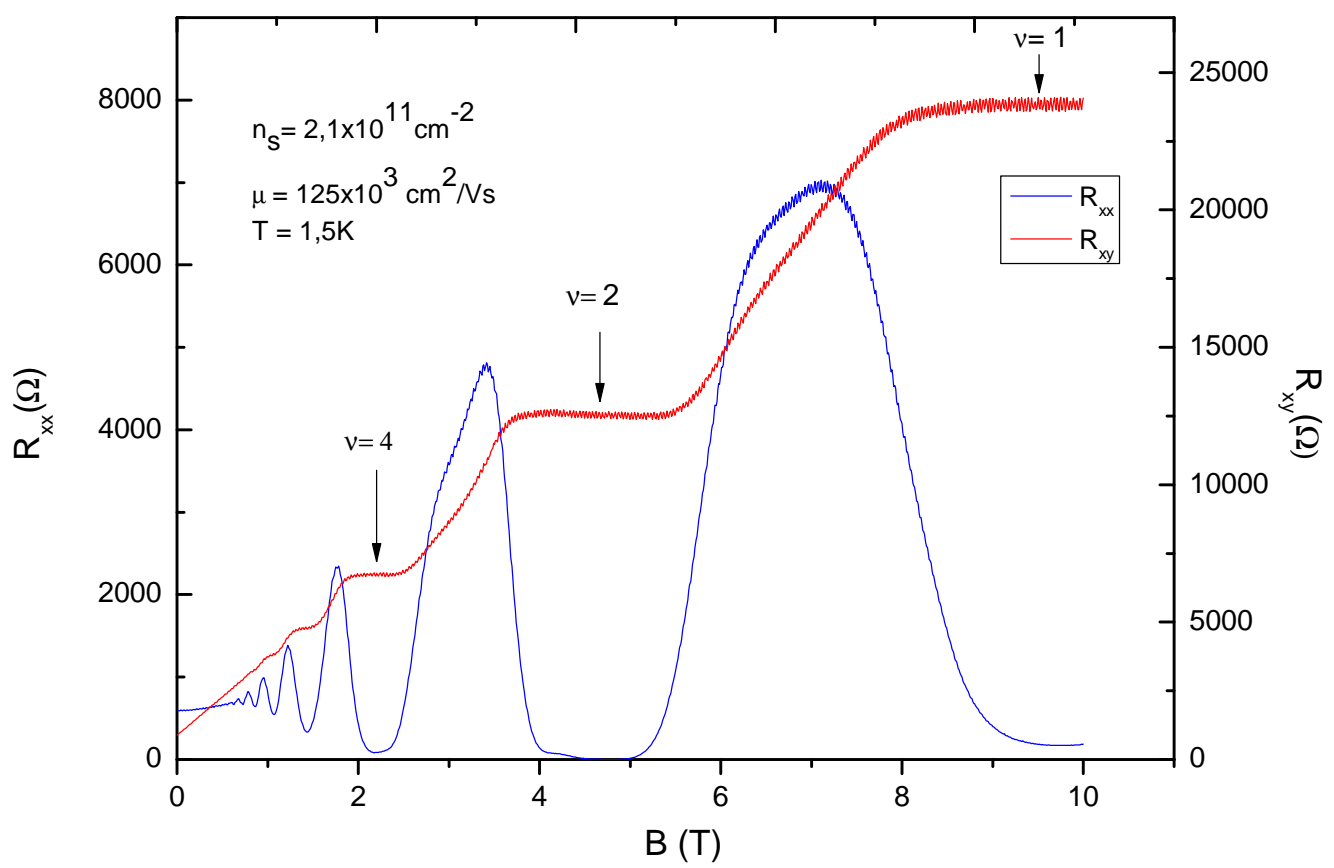

Figura 4.1: Oscilações da magnetoresistência $\left(R_{x x}\right)$ e resistência de Hall $\left(R_{x y}\right)$ para a amostra A2578, a uma temperatura de $1,5 \mathrm{~K}$.

Os elétrons mostram um comportamento do efeito Hall quântico convencional, com platôs largos com uma resistência Hall $h / \nu e^{2}$ acompanhados por mínimos na magnetoresistência. Na figura mostramos também o fator de preenchimento dos níveis de Landau, calculados usando a Eq.(1.45).

Observe-se que para campos baixos (B menor a 0,5T aproximadamente) temos um comportamento linear da resistência de Hall $\left(R_{x y}\right)$. Através da Eq.(1.42), o valor da con- 
centração de Hall ou densidade total de carga é $n_{s}=2,1 \times 10^{11} \mathrm{~cm}^{-2}$. A partir das oscilações da magnetoresistência a campos baixos (oscilações Shubnikov-de Haas), conforme visto na Fig.(4.2a), é possível confirmar o valor da densidade de carga determinando o período dessas oscilações em função da inversa do campo magnético (Fig.4.2b) e usando a Eq.(1.52), obtendo $n_{s}=2,0 \times 10^{11} \mathrm{~cm}^{-2}$.

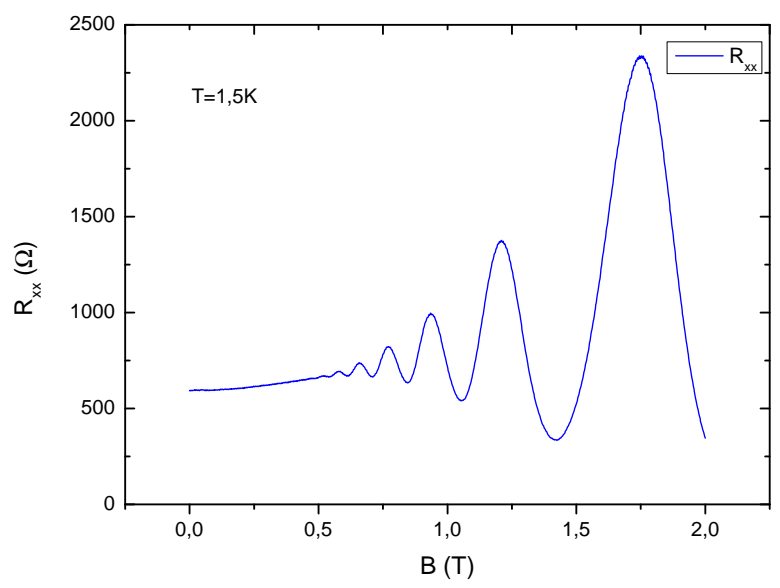

(a)

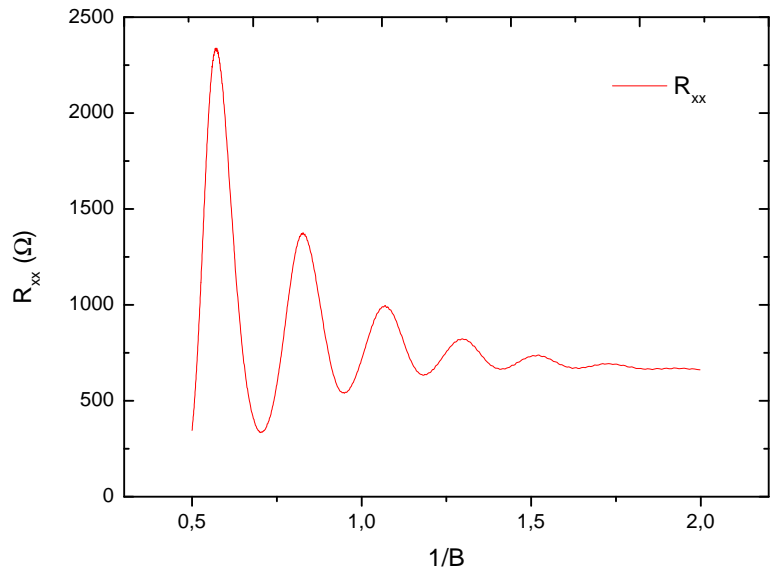

(b)

Figura 4.2: (a) Oscilações Shubnikov-de Haas da amostra A2578. (b) Oscilações Shubnikov-de Haas em função do inverso do campo magnético, elas são periodicas.

Usando os mínimos de magnetoresistência a campos magnéticos altos também é possível obter a concentração de carga através da Eq.(1.49). O valor encontrado é $n_{s}=2,0 \times 10^{11}$ $\mathrm{cm}^{-2}$.

Desta forma, em toda a região de campo magnético, o valor de $n_{s}$ é praticamente o mesmo. Isso indica que a carga capturada pelo poço foi apenas suficiente para preencher 
a primeira sub-banda. Considerando a concentração total $n_{s}=2,1 \times 10^{11} \mathrm{~cm}^{-2}$, é importante calcular a mobilidade dos portadores de carga $\mu$. Para isso, precisamos o valor da resistividade longitudinal a campo magnético zero, descrito no capítulo anterior, que no nosso caso é $\rho_{x x}(0)=239,08 \Omega$. Usando a Eq.(3.4) com os valores de $e=1,6 \times 10^{-19}$ e o valor de $n_{s}$, obtemos que a mobilidade para essa amostra é: $\mu=125 \times 10^{3} \mathrm{~cm}^{2} / V s$.

Uma outra maneira simples e direta de obtermos cada uma das concentrações $n_{s}^{(i)}$ associadas a cada sub-banda ocupada do gás bidimensional, é fazendo a transformada de Fourier (Fast Fourier Transform, FFT) da função $R_{x x}^{\prime \prime}(1 / B)$, onde $1 / B$ é tomado como domínio da função.

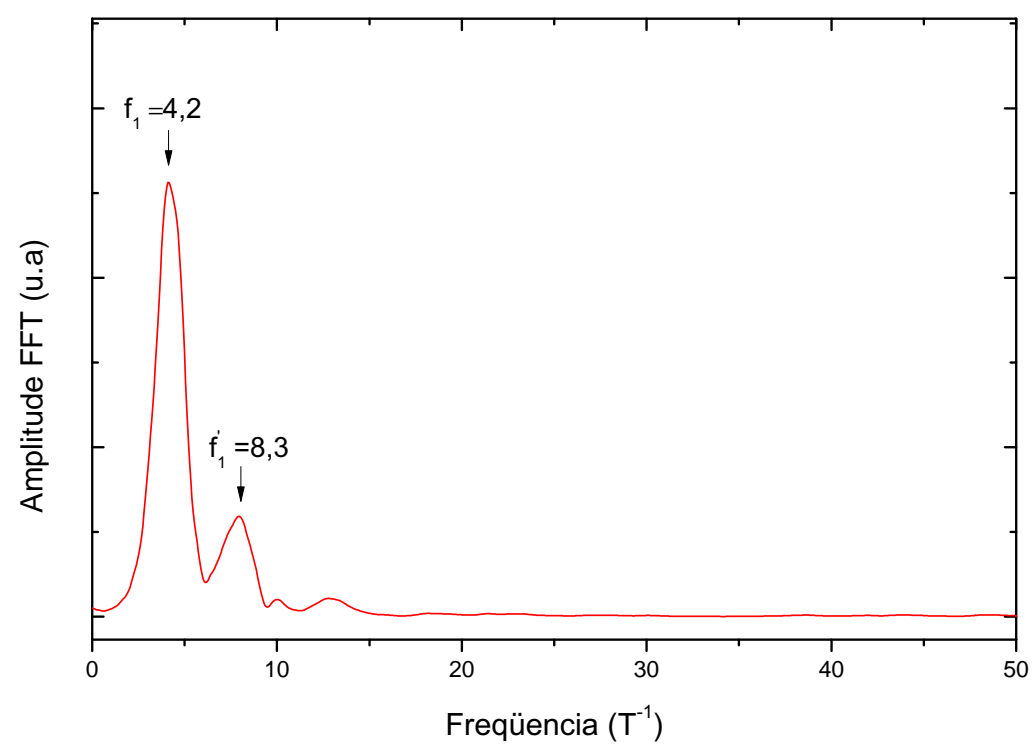

Figura 4.3: Espectro de frequências da transformada de Fourier das oscilações Shubnikov-de Haas da amostra A2578.

Portanto, uma vez tendo os traços das oscilações SdH, o passo seguinte da análise consiste em efetuar a transformada de Fourier dos gráficos $R_{x x}^{\prime \prime} \times 1 / B$, sendo $R_{x x}^{\prime \prime}$ a derivada segunda da magnetoresistência com relação ao recíproco do campo magnético. A descrição detalhada do procedimento é explicado em L. G. Armas [80]. Fizemos isto através do algorítmo FFT disponibilizado pelo software de análise Origin 8.6.

Assim obteve-se um espectro de frequência como o apresentado na Fig.(4.3). A frequência $f_{i}$ do pico na curva da transformada de Fourier está relacionada com a ocupação de cada sub-banda através da relação [81]:

$$
f_{i}(1 / B)=\frac{h n_{s}^{(i)}}{2 e}
$$


Substituindo os valores das constantes obtemos:

$$
n_{s}^{(i)}=0,04836 f_{i}
$$

onde $\left[n_{s}^{(i)}\right]=10^{12} \mathrm{~cm}^{-2}$ e $\left[f_{i}\right]=T^{-1}$.

Assim, no caso em que o gás bidimensional está distribuído em mais de uma sub-banda, observam-se tantas frequências quantas forem as sub-bandas ocupadas. A densidade total de elétrons é determinada somando-se a densidade de cada uma das sub-bandas ocupadas, $n_{s}=\sum_{i} n_{s}^{(i)}$.

Na Fig.(4.3) mostra-se a transformada de Fourier das oscilações SdH medida para a amostra A2578. Nesse espectro de frequência observa-se dois picos principais marcados como $f_{1}$ e $f_{1}^{\prime}$. O primeiro deles indica a ocupação da primeira sub-banda, enquanto o segundo pico representa o seu harmônico. O pico com $f_{1}^{\prime}$ não indica a presença de uma outra sub-banda, e isto nós podemos justificar assim: Suponhamos que associa-se a este pico uma sub-banda ocupada. Através dos espectros apresentados na Fig.(4.3) verificamos que aproximadamente $f_{1}^{\prime}=2 f_{1}$. Portanto, temos que $f_{1}^{\prime}=0$ quando $f_{1}=0$, indicando que ambas sub-bandas começam a ser populadas simultaneamente. Mas isto só é possível quando $E_{1}^{\prime}=E_{1}$ ( $E_{i}$ é a energia do fundo da i-ésima sub-banda), o que não é permitido, pois $E_{i}$ são os autovalores do poço quântico.

$\mathrm{Na}$ verdade, os harmônicos tem origem na conjugação do caráter discreto das transformadas de Fourier com fenômenos falsos tais como ruídos, flutuações de temperatura, etc [82]. Estes harmônicos são frequentemente encontrados e já foram relatados em vários trabalhos, tais como [83] e [84]. Então, iremos ignorá-los, porque eles não trazem nenhuma informação adicional.

Deste modo, a presença do primeiro pico na Fig.(4.3) indica a ocupação de uma única sub-banda para a amostra A2578. Usando a Eq.(4.2) obtem-se que a concentração dessa sub-banda é $n_{s}=2,0 \times 10^{11} \mathrm{~cm}^{-2}$.

No Capítulo 2, fizemos os cálculos autoconsistentes para este poço parabólico utilizando como concentração eletrônica total aquela obtida da resistência de Hall a baixos campos magnéticos: $n_{s}=2,1 \times 10^{11} \mathrm{~cm}^{-2}$. A Tabela(2.4) contém estes resultados, onde mostra-se que o poço parabólico da amostra A2578 tem duas sub-bandas ocupadas, mas a segunda sub-banda mostra uma concentração de portadores quase desprezível, ou seja, encontra-se quase vazia. Por causa disso na nossa análise de Fourier não foi possível encontrar um segundo pico que mostre a ocupação de uma segunda sub-banda. 


\subsubsection{Resultados para a amostra A2580}

Apresentamos agora os resultados correspondentes à amostra A2580 contendo um poço quântico parabólico de largura $W=1000 \AA$. Na Fig.(4.4) mostra-se as medidas de magnetoresistência e resistência de Hall para essa amostra.

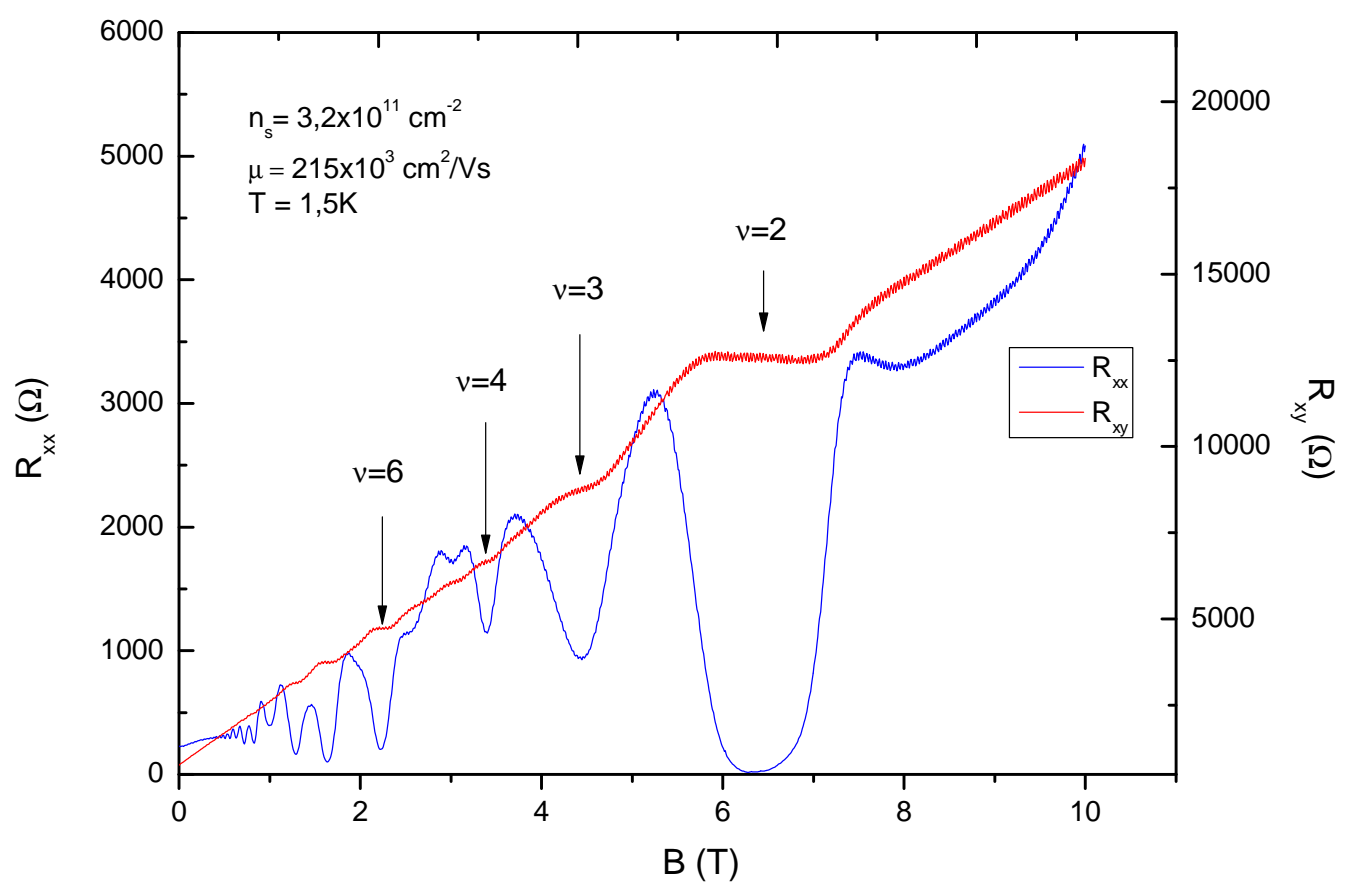

Figura 4.4: Oscilações da magnetoresistência $\left(R_{x x}\right)$ e resistência de Hall $\left(R_{x y}\right)$ para a amostra A2580, a uma temperatura de 1,5K.

Nesse caso, as oscilações da magnetoresistência mostram um comportamento diferente ao das oscilações mostradas na Fig.(4.1). Isso é devido a que na amostra A2580 temos a ocupação de mais de uma sub-banda.

Da região de comportamento clássico da resistência de Hall, através da Eq.(1.42), o valor da concentração de Hall é $n_{s}=3,2 \times 10^{11} \mathrm{~cm}^{-2}$. É esse valor que foi utilizado para fazer os cálculos autoconsistentes para essa amostra.

A Fig.(4.5a) mostra as oscilações Shubnikov-de Haas para essa amostra, de onde é determinada uma densidade de carga $n_{s}=2,4 \times 10^{11} \mathrm{~cm}^{-2}$ para campos baixos. Mas dos mínimos da magnetoresistência a campos altos obtemos uma densidade de portadores $n_{s}=3,1 \times 10^{11} \mathrm{~cm}^{-2}$. 


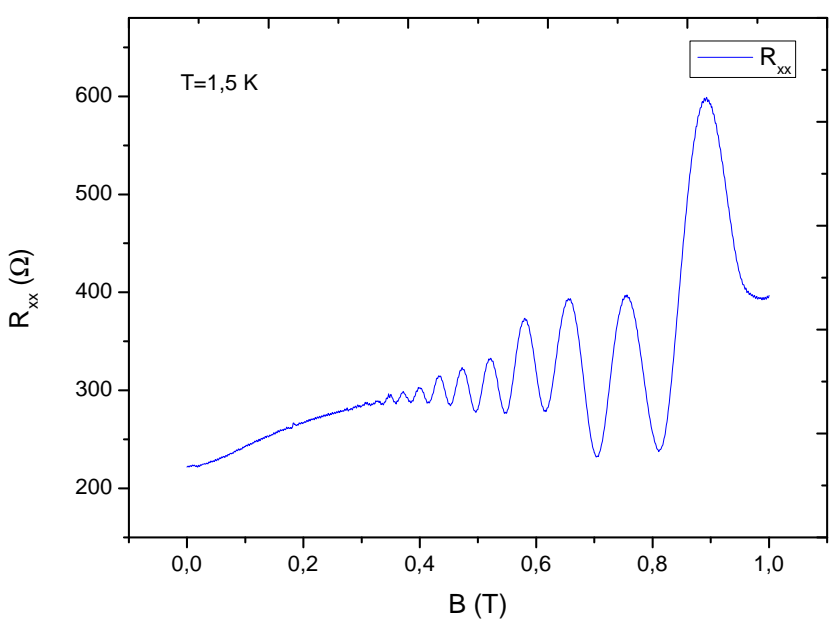

(a)

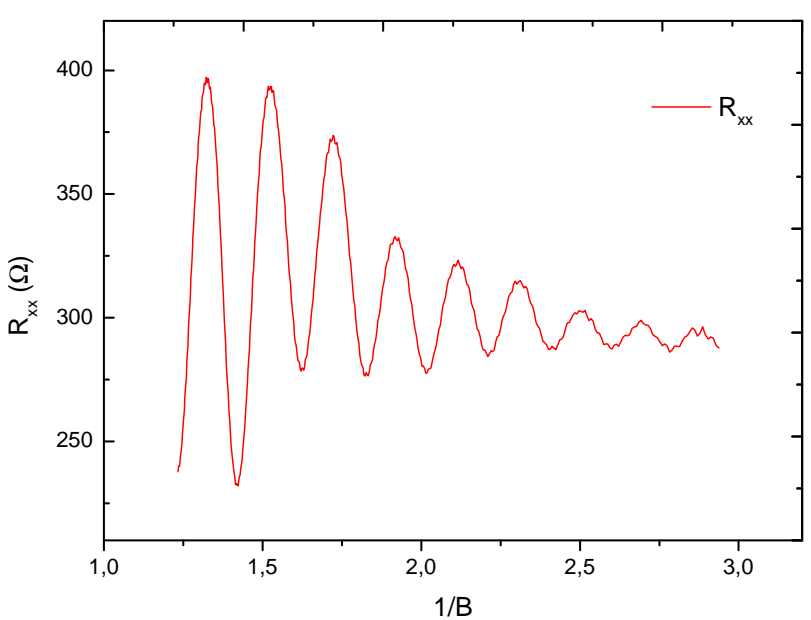

(b)

Figura 4.5: (a) Oscilações Shubnikov-de Haas da amostra A2580. (b) Oscilações Shubnikov-de Haas em função do inverso do campo magnético, elas são periodicas.

Observa-se nesse caso, que a densidade de portadores obtida na região de campos magnéticos baixos é diferente à extraída em campos altos. Este fato indica que o poço quântico possui mais de uma sub-banda ocupada. Baseando-se nos cálculos autoconsistentes para este poço, mostra-se que ele contém duas sub-bandas ocupadas (ver Capítulo 2). Assim, atribuímos o valor a campo baixo como sendo proveniente das oscilações da primeira sub-banda ocupada, e o valor a campo alto como sendo a carga total capturada pelo poço.

Usando a Eq.(3.4), determinou-se o valor da mobilidade da amostra a partir do valor da concentração total $n_{s}=3,2 \times 10^{11} \mathrm{~cm}^{-2}$ e do valor da resistividade longitudinal a 
campo magnético zero $\rho_{x x}(0)=91,32 \Omega$. Assim, a mobilidade para a amostra A2580 é $\mu=214 \times 10^{3} \mathrm{~cm}^{2} / V s$.

Os procedimentos para encontrar as concentrações de cada sub-banda ocupada foram os mesmos que para a amostra A2578. Usando os traços das oscilações Shubnikov-de Haas das figuras (4.5a) e (4.5b) e fazendo o análise da transformada de Fourier FFT, obteve-se o espectro de frequências mostrado na Fig.(4.6).

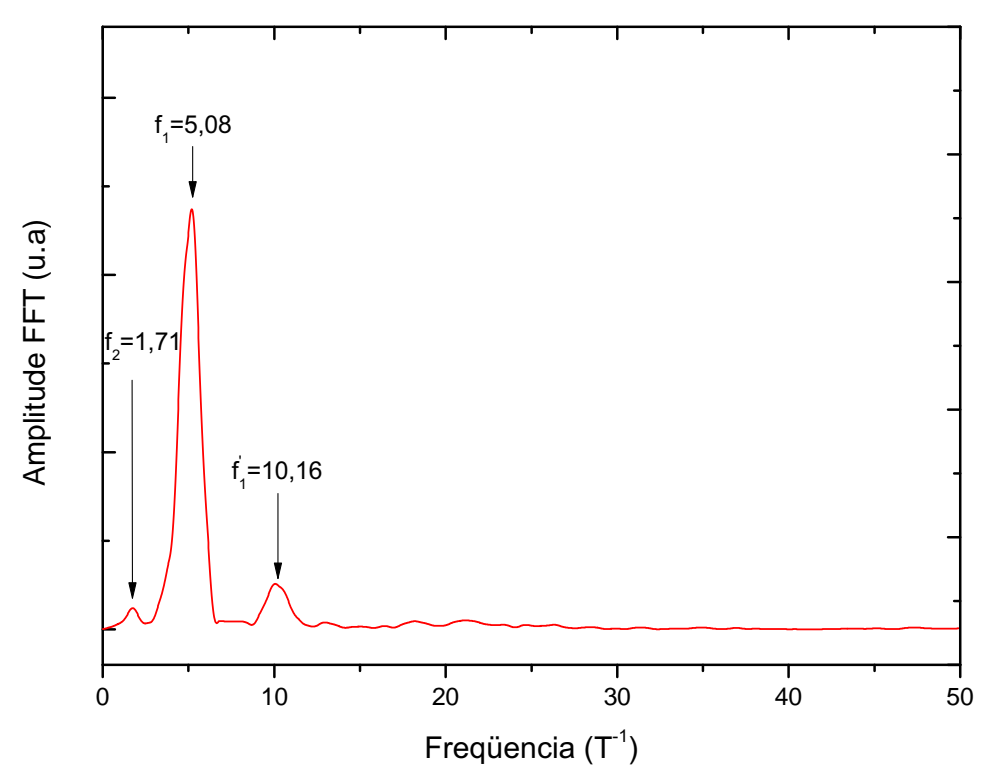

Figura 4.6: Espectro de frequências da transformada de Fourier das oscilações Shubnikov-de Haas da amostra A2580.

Nessa figura observa-se dois picos marcados como $f_{1}$ e $f_{2}$ que indicam a ocupação da primeira e segunda sub-banda respectivamente. Também aparece um terceiro pico marcado como $f_{1}^{\prime}$ que corresponde ao harmônico da primeira sub-banda, pois a sua frequência é exatamente o dobro da frequência correspondente à primeira sub-banda.

Deste modo, usando a Eq.(4.2) e as frequências encontradas no espectro da Fig.(4.6), obtem-se que as concentrações em cada uma das sub-bandas são: $n_{s}^{(1)}=2,45 \times 10^{11} \mathrm{~cm}^{-2}$ para a primeira sub-banda, e $n_{s}^{(2)}=0,82 \times 10^{11} \mathrm{~cm}^{-2}$ para a segunda sub-banda.

Os valores das concentrações obtidas através da transformada de Fourier estão em concordância com os resultados obtidos dos cálculos autoconsistentes. Isso é mostrado na Tabela(2.5) do Capítulo 2. 


\subsection{Medidas em poços quânticos parabólicos com porta com barreira}

Neste caso estudou-se a amostra A2519 contendo um poço quântico parabólico de largura $W=3000 \AA$, densidade de carga $n_{s}=1,9 \times 10^{11} \mathrm{~cm}^{-2}$ e mobilidade $\mu=220 \times 10^{3}$ $\mathrm{cm}^{2} /$ Vs. Nesta amostra foram feitas medidas de magnetotransporte nas configurações de campo magnético perpendicular e campo paralelo, aplicando diferentes voltagens de porta. A porta metálica com barreira feita na superfície da amostra nos levou a estudar os fenômenos de transporte através do modelo de transporte de estados de borda [85].

Através da aplicação de uma tensão $V_{g}$ na porta, a concentração de portadores na região da barreira (única região afetada pelo potencial aplicado) pode ser alterada. Em campos magnéticos intensos, a densidade de estados se divide em níveis de Landau e de spin com uma degenerescência de $n_{\text {deg }}=e B / h$ por nível. Assim, mudando a concentração de portadores na região da barreira, o fator de preenchimento dessa região é alterado e a amostra conterá regiões com fatores de preenchimento diferentes.

No modelo de estados de borda, o fator de preenchimento esta conectado ao número de estados de borda ocupados. Através da aplicação de uma voltagem negativa na porta, é possível depletar a região da barreira, isto é, diminuir o número de estados de borda nesta região. Assim, os estados de borda que existem nas regiões não perturbadas adjacentes, são refletidas na região da barreira. Isto é mostrado esquematicamente na Fig.(4.7).

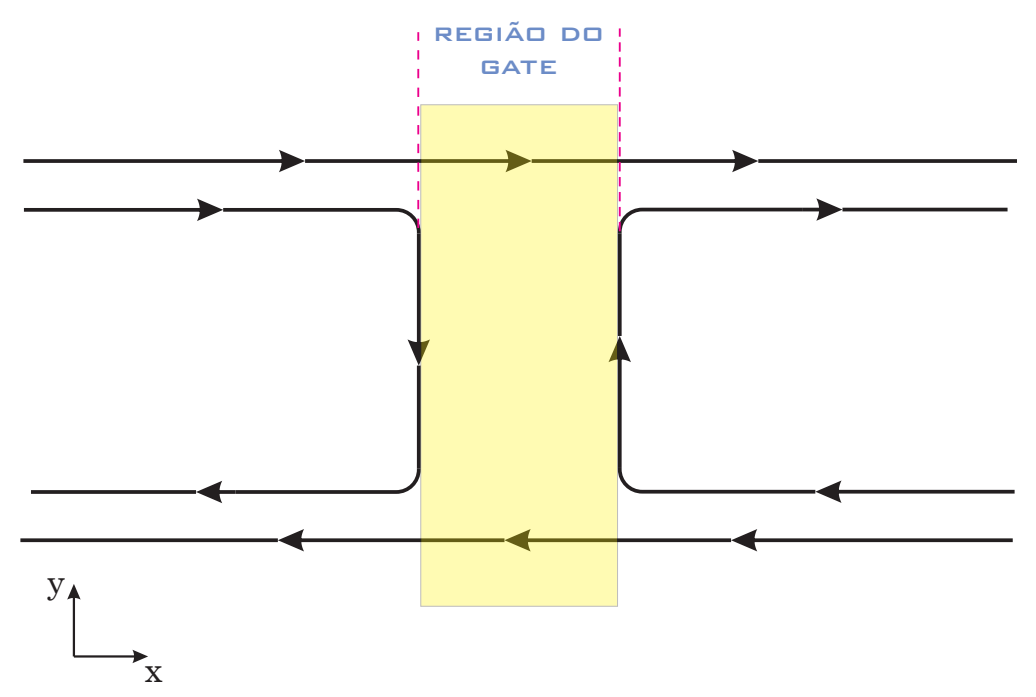

Figura 4.7: Esquema do modelo de estados de borda para uma amostra com barreira. Nesse caso, é aplicada uma tensão de porta negativa $\left(V_{g}<0\right)$ que gera uma reflexão dos estados de borda mais internos na barreira. Nas regiões não perturbadas temos fator de preenchimento $\nu=2$, e na região da barreira temos $\nu_{g}=1$. 
Dentro do modelo de estados de borda, é possivel calcular os valores de resistência com a ajuda da fórmula de Landauer-Buttiker. Assim, sendo $\nu_{g}$ o fator de preenchimento na região da porta, e $\nu$ o fator de preenchimento na região não perturbada da amostra, os valores de resistência nos platôs que aparecem na magnetoresistência são dados pela equação [38]:

$$
R_{x x}=\frac{h}{e^{2}}\left(\frac{1}{\nu_{g}}-\frac{1}{\nu}\right)
$$

Primeiramente, foram feitas medidas em configuração de campo magnético perpendicular. Nessa configuração, na amostra A2519 é possível observar que a magnetoresistência apresenta um comportamento muito bem descrito pelo modelo de estados de borda, onde temos o aparecimento de uns platôs que obedecem à Eq.(4.3). Usando a relação $\nu=n_{s} h / e B$, onde $\nu$ é o fator de preenchimento [38], é possível determinar o fator de preenchimento na região da porta $\nu_{g}$.

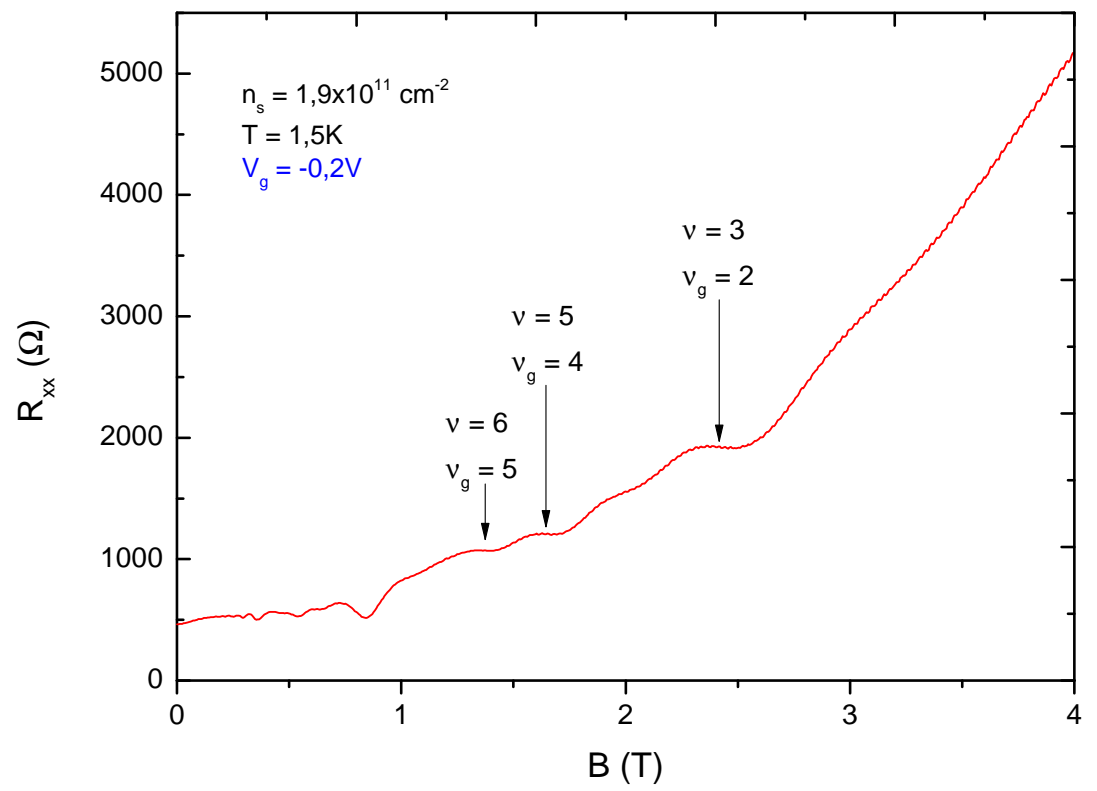

Figura 4.8: Magnetoresistência na amostra A2519 para uma voltagem de porta $V_{g}=-0,2 \mathrm{~V}$ em campo magnético perpendicular.

A Fig.(4.8) mostra três platôs, indicados por umas setas pretas, na magnetoresistência para uma voltagem de porta $V_{g}=-0,2 V$. Para esses valores de campos magnéticos temos que é satisfeita a Eq.(4.3), dando como resultado os mostrados na figura. Em todos esses platôs pode-se concluir que o canal de borda mais interno pertencente ao nível de Landau mais alto, é totalmente refletido. 
Da mesma maneira, a Fig.(4.9) apresenta um platô na magnetoresistência para uma voltagem de porta $V_{g}=-0,4 \mathrm{~V}$. Nesse caso também foi possível encontrar valores inteiros para o fator de preenchimento na região da porta e na região não perturbada pela tensão de porta.

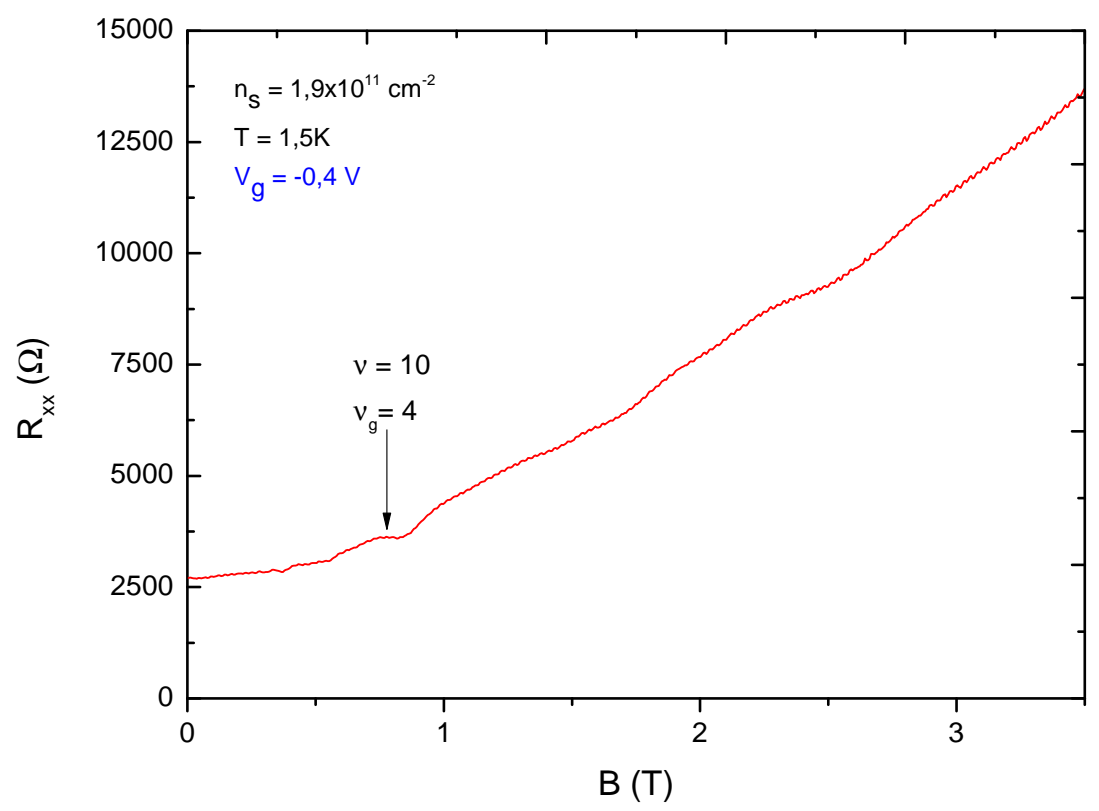

Figura 4.9: Magnetoresistência na amostra A2519 para uma voltagem de porta $V_{g}=-0,4 \mathrm{~V}$ em campo magnético perpendicular.

Também realizou-se medidas de magnetoresistência em configuração de campo magnético paralelo. Nesse caso, as medidas foram feitas fixando o valor do campo magnético enquanto variava-se a tensão de porta desde $0,0 \mathrm{~V}$ até $-0,8 \mathrm{~V}$.

Na Fig.(4.10) mostra-se as medidas da magnetoresistência $\left(R_{x x}\right)$ em função da voltagem de porta $\left(V_{g}\right)$ para diferentes valores de campos magnéticos fixos. Devido ao aumento súbito da magnetoresistência, é conveniente expressar esse gráfico em uma escala logarítmica.

Com as medidas da Fig.(4.10) é possível fazer um gráfico tridimensional da magnetoresistência em função da voltagem de porta e do campo magnético $B$. Esse gráfico 3D é mostrado na Fig.(4.11a), onde a magnetoresistência é expressa em uma escala linear, e mostra-se também na Fig.(4.11b) em uma escala logarítmica. 


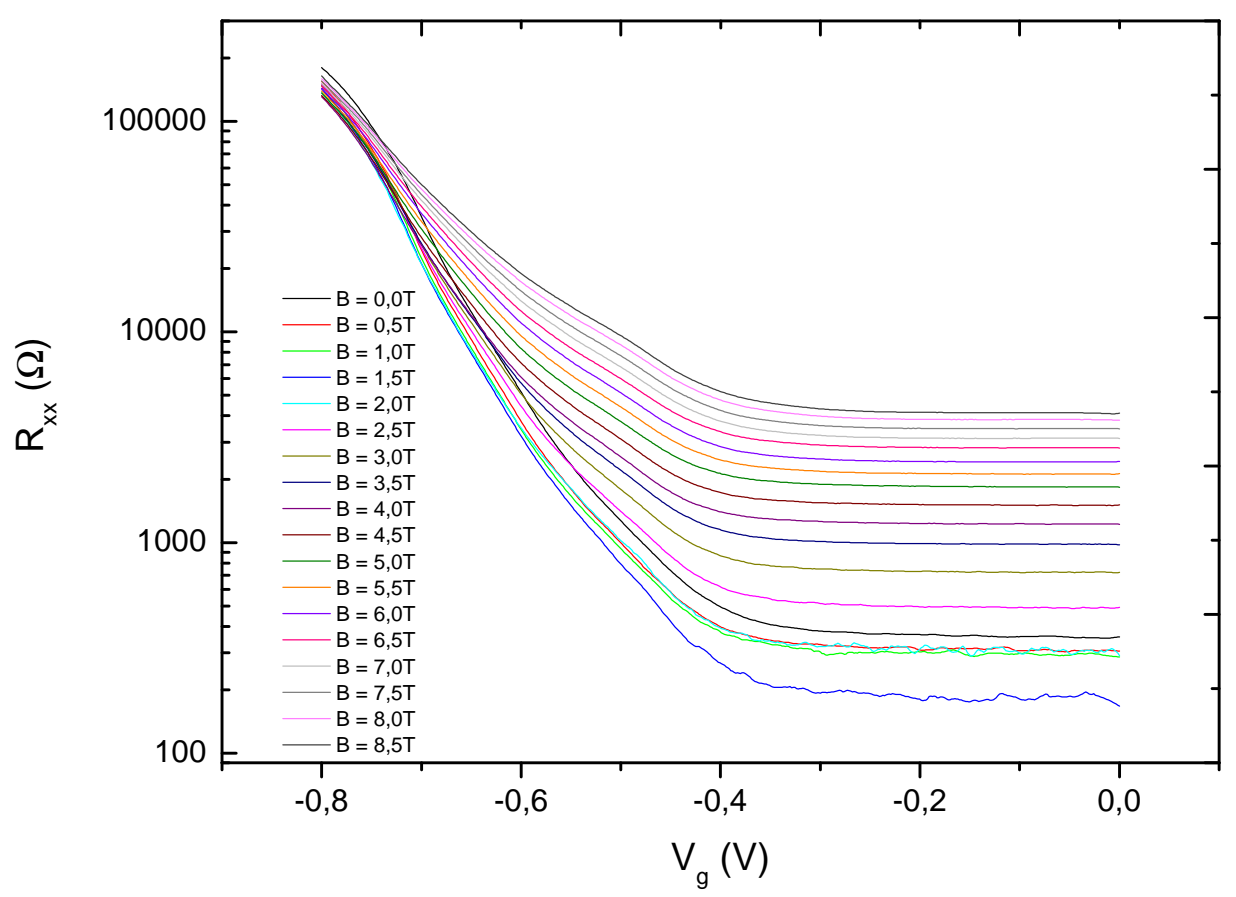

Figura 4.10: Magnetoresistência da amostra A2519 em função da voltagem de porta para diferentes valores de campo magnético. O gráfico está em escala logarítmica.

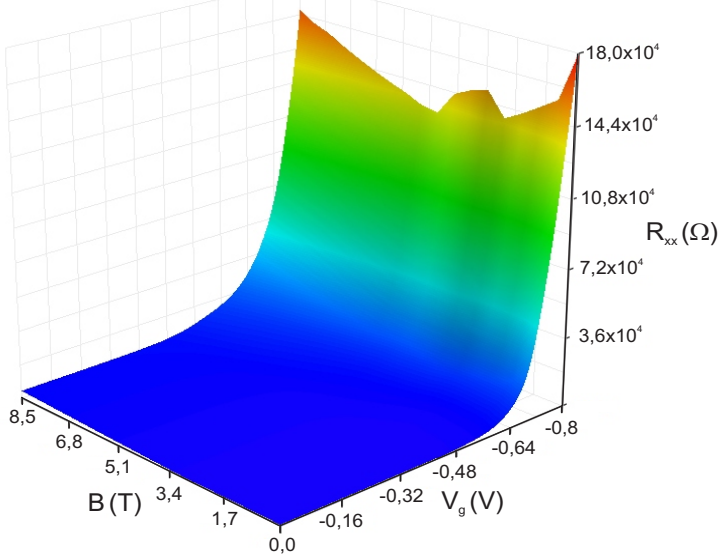

(a)

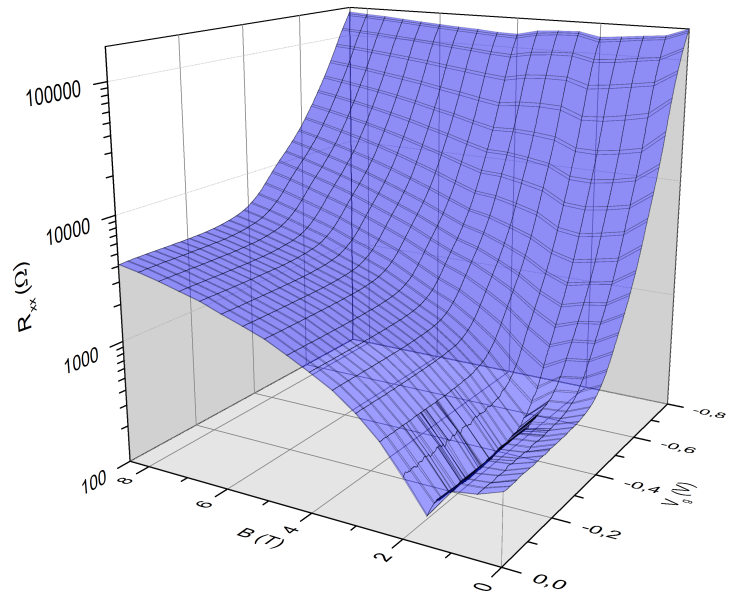

(b)

Figura 4.11: Gráficos tridimensionais da magnetoresistência para a amostra A2519 em função da voltagem de porta $V_{g}$ e do campo magnético B paralelo. (a) Mostra-se o gráfico da magnetoresistência em escala linear. (b) Mostra-se o gráfico da magnetoresistência em escala logarítmica. 
As curvas de magnetoresistência mostradas na Fig.(4.10) apresentam um comportamento de tal forma que é possível ajustar elas a curvas exponenciais. Esse ajuste é feito para cada valor de campo magnético $B$ fixo. A equação usada para o ajuste exponencial de $R_{x x}$ em função de $V_{g}$ é a seguinte:

$$
R_{x x}^{\text {Ajuste }}=R_{0}+a \times e^{b V_{g}}
$$

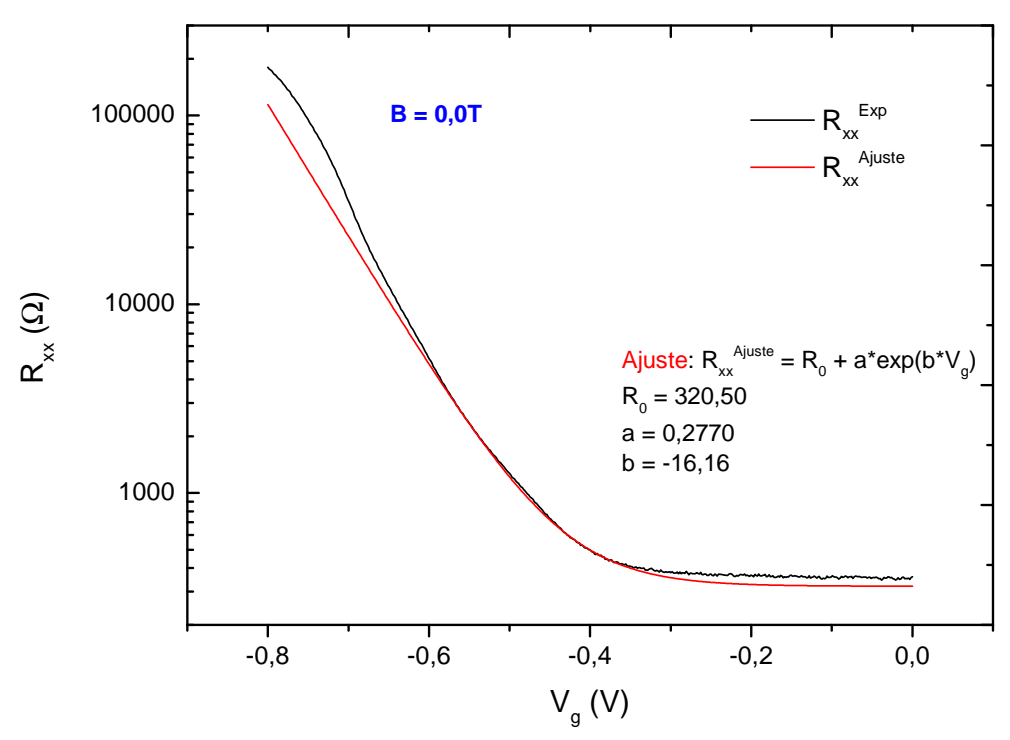

(a)

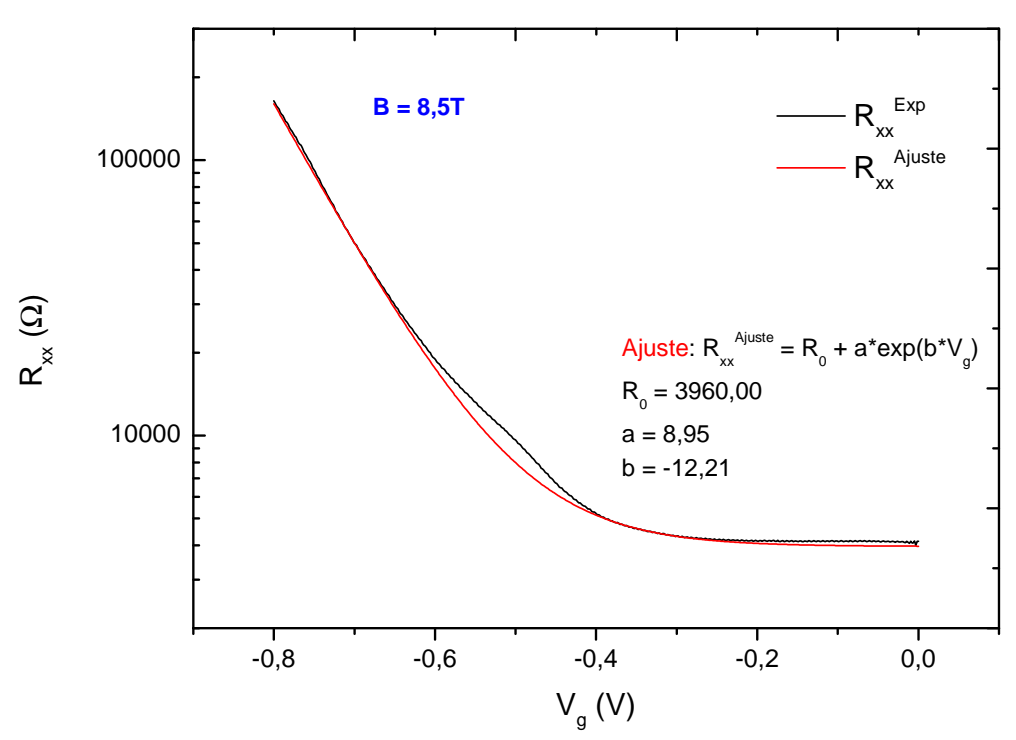

(b)

Figura 4.12: (a) Magnetoresistência $R_{x x}$ e curva ajustada $R_{x x}^{\text {Ajuste }}$ para $B=0,0 T$. (b) Magnetoresistência $R_{x x}$ e curva ajustada $R_{x x}^{A j u s t e}$ para $B=8,5 T$. 
É mostrado como exemplo, os gráficos para dois dos ajustes feitos em campos magnéticos diferentes. Na Fig.(4.12a) mostra-se a magnetoresistência experimental $\left(R_{x x}^{E x p}\right)$ e a magnetoresistência ajustada $\left(R_{x x}^{\text {Ajuste }}\right)$ à Eq.(4.4) para o campo magnético $B=0,0 \mathrm{~T}$, e a Fig.(4.12b) mostra $R_{x x}^{E x p}$ e $R_{x x}^{\text {Ajuste }}$ para o campo magnético $B=8,5 \mathrm{~T}$.

Note-se que os valores ajustados de $R_{0}, a$ e $b$ mudam para cada valor de campo magnético $B$. Assim, do ajuste de todas as curvas de magnetoresistência mostradas na Fig.(4.10) é possível obter a variação dos parâmetros $R_{0}$, $a$ e $b$ com o campo magnético $B$. A Tabela (4.2) mostra os valores de $R_{0}, a$ e $b$ para cada valor de campo magnético $B$.

\begin{tabular}{c|c|c|c}
\hline \hline $\mathrm{B}(\mathrm{T})$ & $R_{0}$ & $a$ & $b$ \\
\hline \hline 0,0 & 320,5 & 0,277 & $-16,16$ \\
0,5 & 300,0 & 0,085 & $-17,67$ \\
1,0 & 287,5 & 0,061 & $-18,08$ \\
1,5 & 176,5 & 0,054 & $-18,21$ \\
2,0 & 300,0 & 0,068 & $-17,90$ \\
2,5 & 485,0 & 0,113 & $-17,44$ \\
3,0 & 710,0 & 0,115 & $-17,07$ \\
3,5 & 970,0 & 0,195 & $-16,79$ \\
4,0 & 1210,0 & 0,256 & $-16,37$ \\
4,5 & 1481,0 & 0,417 & $-15,79$ \\
5,0 & 1800,0 & 0,675 & $-15,23$ \\
5,5 & 2068,0 & 1,080 & $-14,68$ \\
6,0 & 2336,0 & 1,737 & $-14,12$ \\
6,5 & 2700,0 & 2,540 & $-13,68$ \\
7,0 & 3000,7 & 3,680 & $-13,24$ \\
7,5 & 3317,0 & 5,050 & $-12,88$ \\
8,0 & 3648,0 & 6,820 & $-12,53$ \\
8,5 & 3960,0 & 8,950 & $-12,21$ \\
\hline \hline
\end{tabular}

Tabela 4.2: Mostra-se os valores dos parâmetros $R_{0}$, a e b para cada campo magnético $B$.

Os valores dos parâmetros $R_{0}, a$ e $b$ da Tabela (4.2) podem ser mostrados graficamente. A Fig.(4.13) mostra como varia o parâmetro $R_{0}$ em função de $B$. Observa-se que para $B>1,5 \mathrm{~T}$ aproximadamente, temos um comportamento linear, assim na mesma figura também é mostrado o ajuste linear válido só para $B>1,5 \mathrm{~T}$. A equação que descreve o comportamento de $R_{0}$ na região linear é dada por:

$$
R_{0}^{\text {Ajuste }}=580,0 \times B-1100 ; \quad B>1,5 T
$$




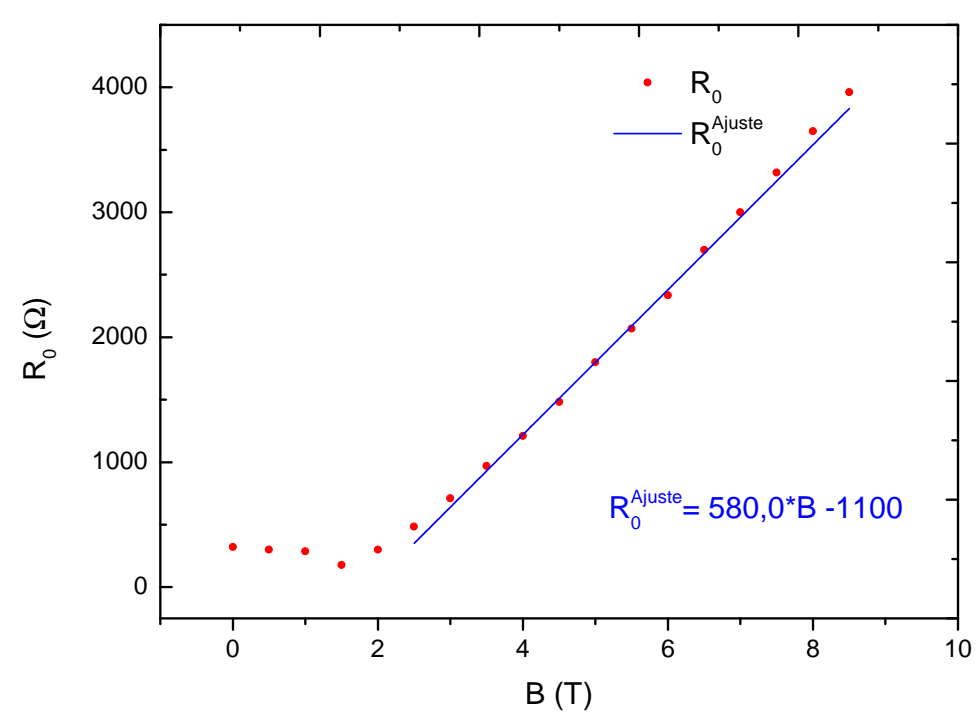

Figura 4.13: Variação de $R_{0}$ com o campo magnético. Mostra-se também o ajuste linear para a região $B>1,5 T$.

Na Fig.(4.14) mostra-se graficamente a variação do parâmetro a com o campo magnético $B$. Observa-se que nesse caso a variação é exponencial, assim, mostra-se também o ajuste exponencial válido para $B>1,5 \mathrm{~T}$. Esse ajuste é descrito pela equação:

$$
a^{\text {Ajuste }}=0,019 \times e^{(0,74 \times B)} ; \quad B>1,5 T
$$

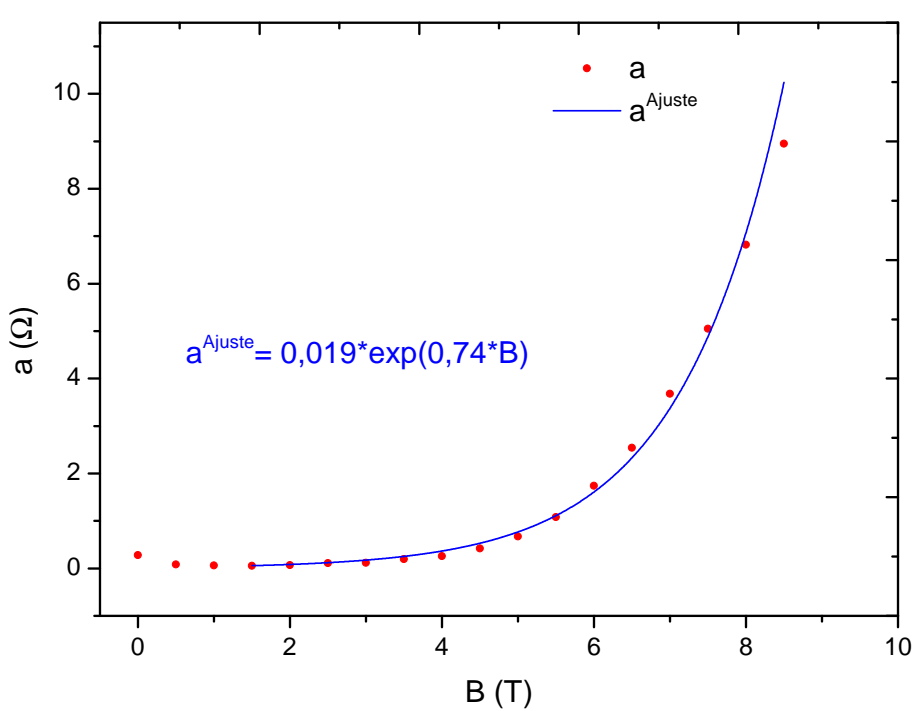

Figura 4.14: Variação de a com o campo magnético. Mostra-se também o ajuste exponencial para a região $B>1,5 T$. 
A variação do parâmetro $b$ com o campo magnético é mostrado na Fig.(4.15). Note-se que também é possível observar que na região para $B>1,5 \mathrm{~T}$ o comportamento é linear, e pode descrever-se através da equação:

$$
b^{\text {Ajuste }}=0,91 \times B-19,76 ; \quad B>1,5 T
$$

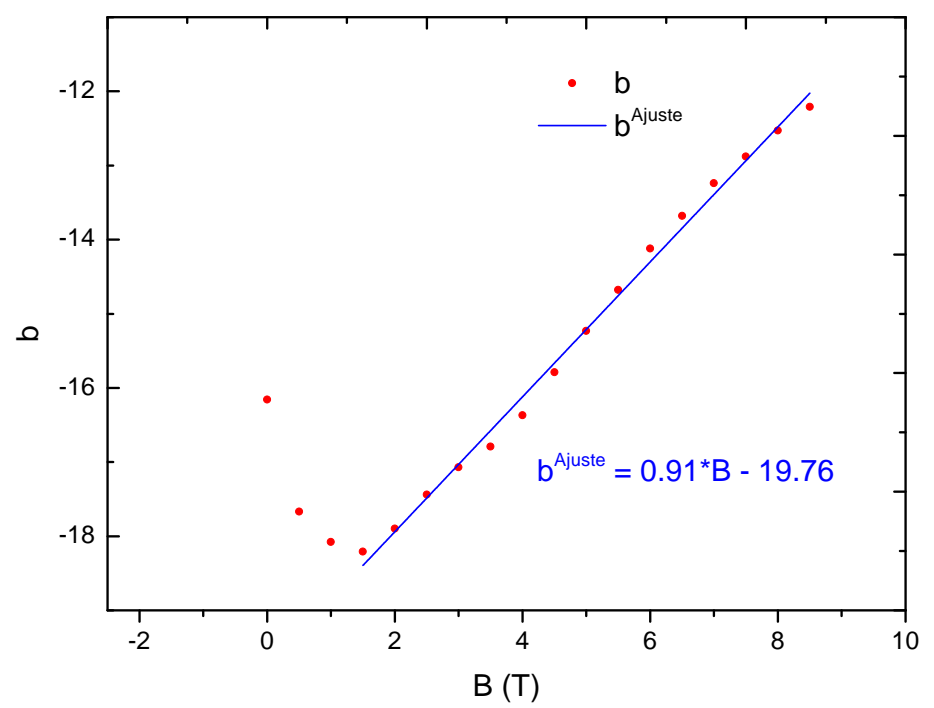

Figura 4.15: Variação de b com o campo magnético. Mostra-se também o ajuste linear para a região $B>1,5 T$.

Dessa maneira, temos expressões aproximadas para os parâmetros $R_{0}, a$ e $b$ em função do campo magnético $B$, validas para $B>1,5 \mathrm{~T}$. Com essas expressões (Eq.(4.5), Eq.(4.6) e Eq.(4.7)), podemos determinar uma equação aproximada para descrever a dependência da magnetoresistência com o campo magnético e a tensão de porta. Assim, substituindo as expressões para $R_{0}(B), a(B)$ e $b(B)$ na equação de ajuste exponencial Eq.(4.4), obtemos a seguinte relação:

$$
R_{x x}\left(B, V_{g}\right)=-1100+580 B+0,019 e^{\left[\left(0,74+0,91 V_{g}\right) B-19,76 V_{g}\right]} ; \quad B>1,5 T
$$




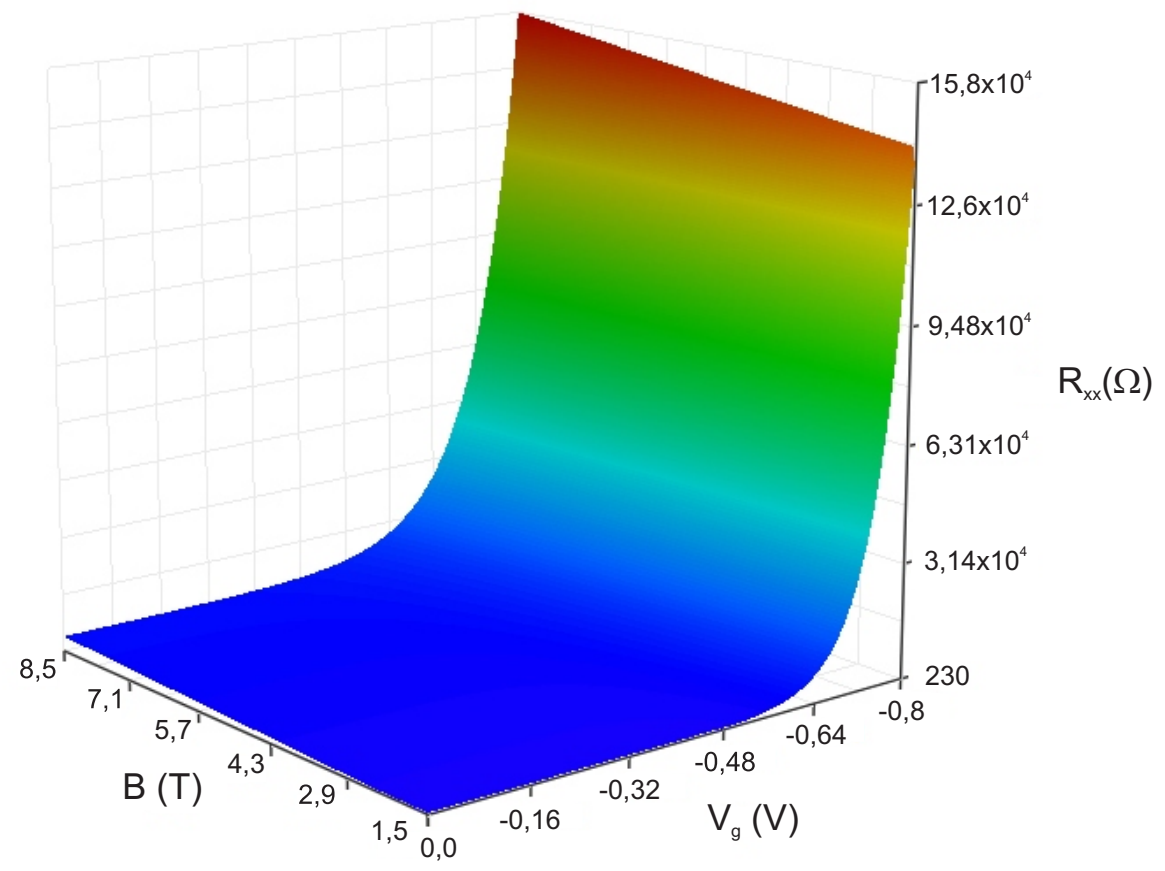

(a)

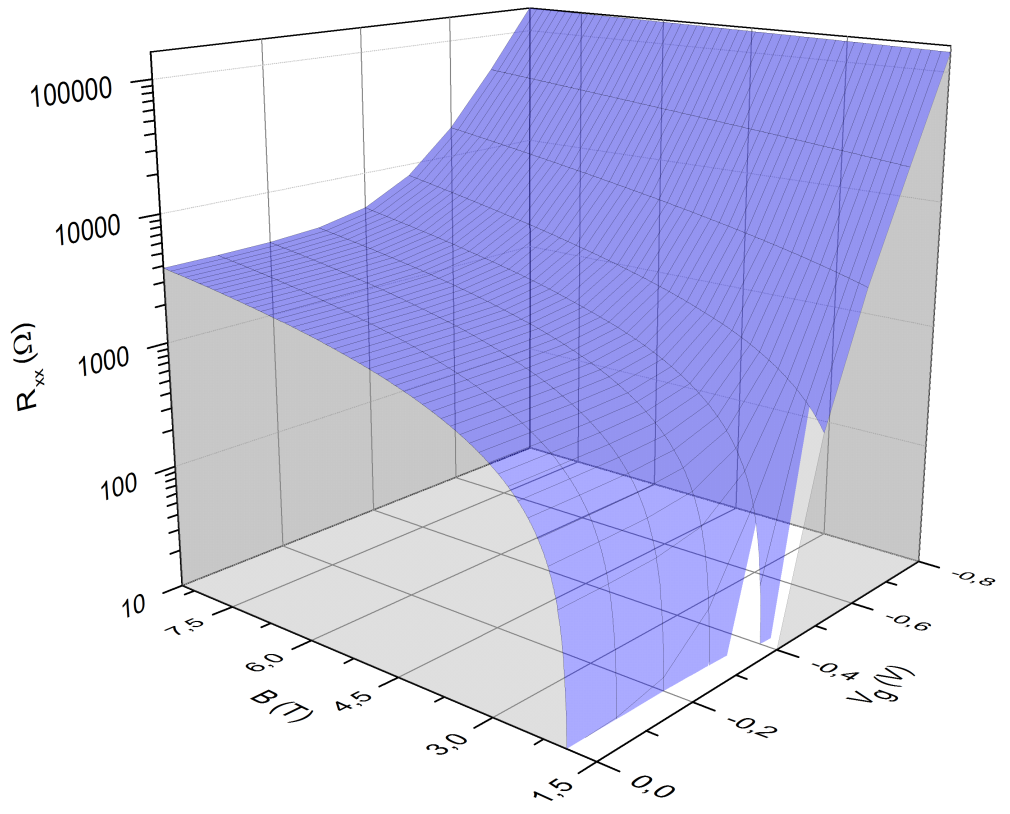

(b)

Figura 4.16: Magnetoresistência em função da tensão de porta e do campo magnético para $B>1,5 T$ obtida a partir da Eq.(4.8). (a) Gráfico em escala linear. (b) Gráfico em escala logarítmica. 
Note-se que na nossa amostra (Amostra A2519), o fator de forma na região da barreira é muito menor do que o fator de forma no canal de corrente da barra Hall (onde $f=2,5$ ), isto, devido a que a barreira tem uma largura de aproximadamente $d=350 \mathrm{~nm}$, como mostra-se na Fig.(4.17). Assim, o fator de forma na barreira é $f=\frac{350 \mathrm{~nm}}{200 \mu \mathrm{m}}=1,75 \times 10^{-3}$.

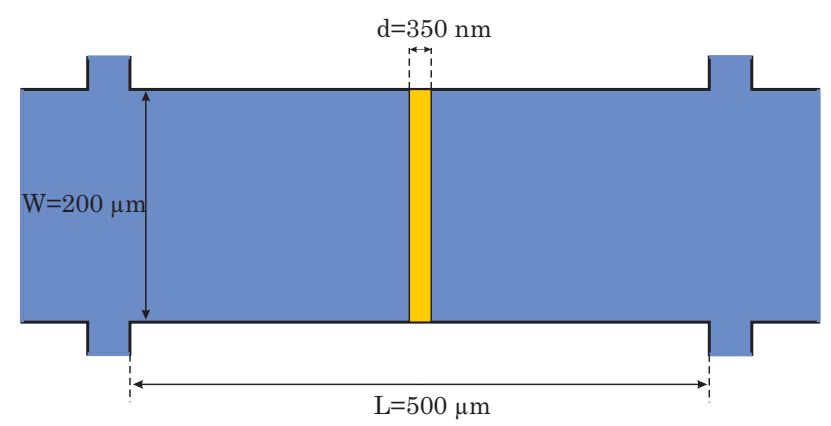

Fator de forma na barreira $: \mathrm{f}=\mathrm{d} / \mathrm{W}=1,75 \times 10^{-3}$

Figura 4.17: Esquema do canal de corrente da barra Hall onde são mostradas as dimensões da barreira (em amarelho). Tal largura da barreira gera um fator de forma $f=1,75 \times 10^{-3}$.

O fato do fator de forma na barreira ser muito pequeno faz que a resistência quadrado $\left(R_{\square}\right)$ nessa região seja muito alta, variando desde $100 \mathrm{~K} \Omega$ até $100 \mathrm{M} \Omega$ dependendo dos valores de campo magnético e da tensão de porta. A resistência quadrado pode ser calculada usando: $R_{\square}=R_{x x} / f$, com $f=1,75 \times 10^{-3}$.

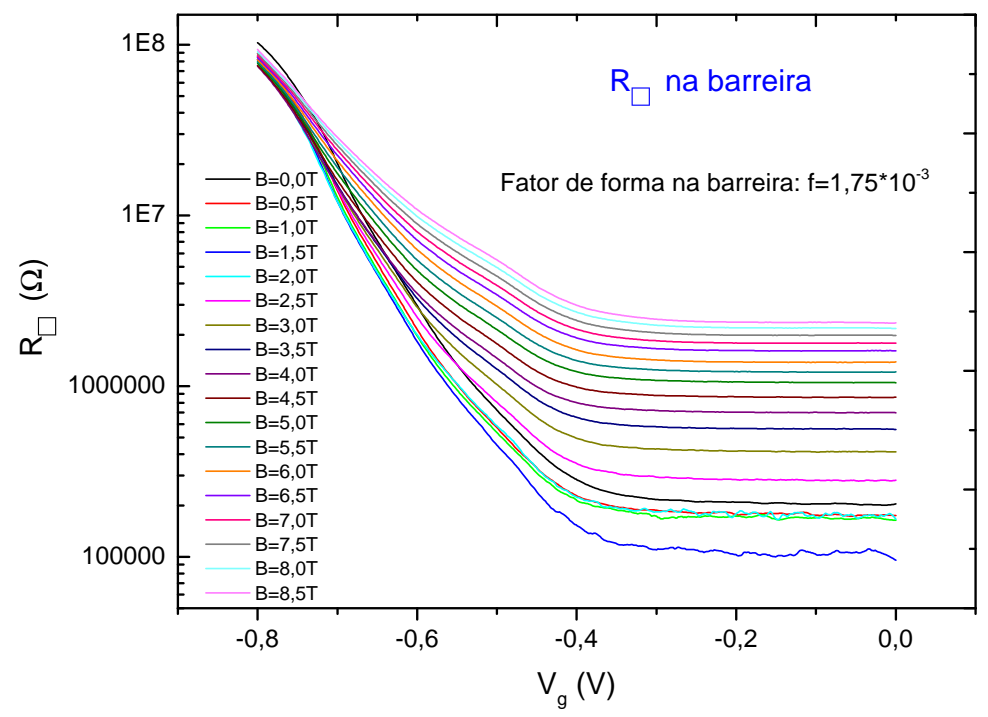

Figura 4.18: Mostra-se a resistência quadrado na região da barreira em função da tensão de porta para diferentes valores de campo magnético. O gráfico está em escala logarítmica. 
Na Fig.(4.18) mostra-se os valores correspondentes da $R_{\square}$ como consecuencia de dividir os valores experimentais da magnetoresistência sobre o fator de forma na barreira. A Fig.(4.19) mostra o gráfico tridimensional dos valores de $R_{\square}$ em função do campo e da tensão de porta.

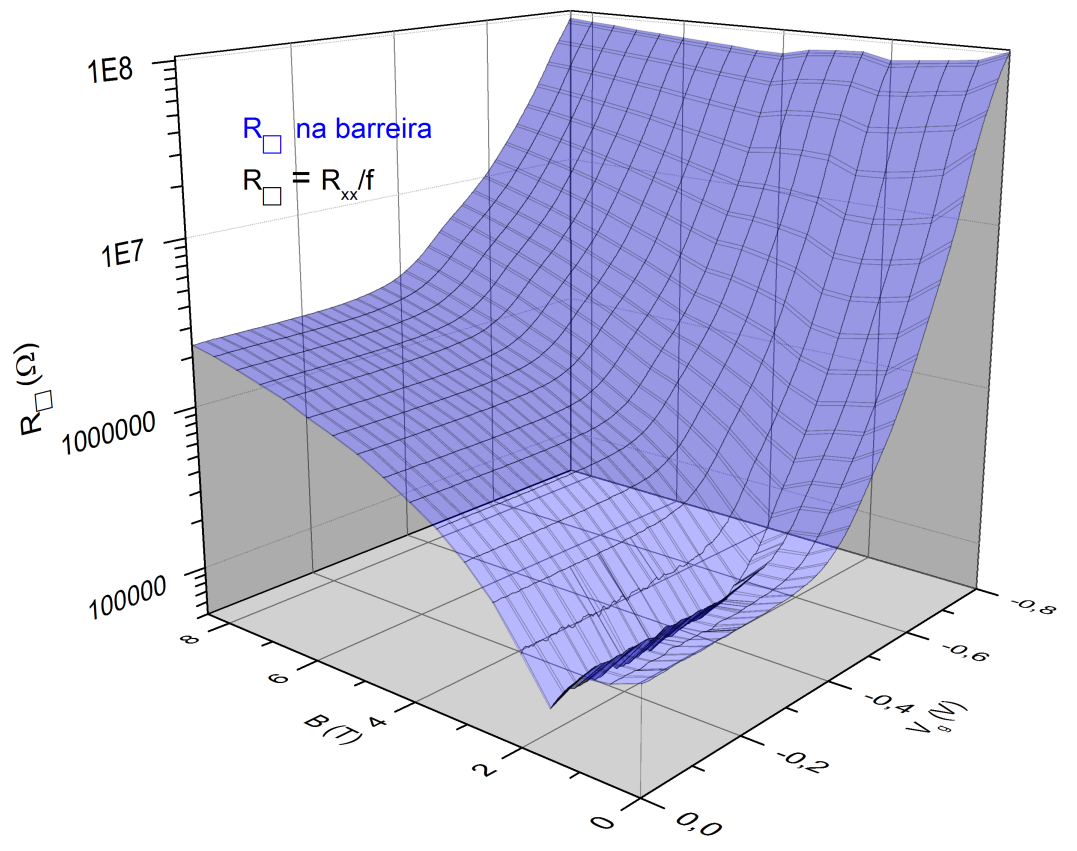

Figura 4.19: Mostra-se o gráfico tridimensional em escala logarítmica da $R_{\square}$ na região da barreira em função do campo (B) e da tensão de porta $\left(V_{g}\right)$.

Observe-se que os valores de $R_{\square}$ na barreira são muito grandes, e crescem subitamente com o incremento da tensão de porta. Isso quer dizer que a tensão de porta atua como uma barreira de potencial, e nessa região a passagem de elétrons se da através de tunelamento nessa barreira de potencial, quando a $V_{g}$ é grande.

As nossas medidas podem ser entendidas desde um ponto de vista semi-clássico, a partir do comportamento ciclotrônico dos elétrons sem levar em conta o spin. Em primeiro lugar, note-se que o nosso sistema na presença de campo magnético paralelo, forma um gás quasetridimensional de elétrons. Isto resulta do fato de que no limite de campos magnéticos elevados, na aproximação semi-clássica, os elétrons descrevem órbitas ciclotrônicas cujo raio varia com $\sqrt{B}$, caracterizadas portanto por um comprimento magnético $l_{B}=\sqrt{\hbar / m^{*} \omega_{c}}=$ $\sqrt{\hbar / e B}$. Para o caso em que o campo magnético é paralelo ao gás de elétrons, a partir de um certo limite de campo, o comprimento magnético $l_{B}$ se aproxima ao valor da largura do poço quântico parabólico, e o caráter ciclotrônico sobressai em relação ao confinamento imposto pelo potencial parabólico. Para um campo de $1 \mathrm{~T}$, temos que $l_{B}=250 \AA$, enquanto que para um campo magnético de $8 \mathrm{~T}$ o valor do comprimento magnético é $l_{B}=90 \AA$. Desse 
modo, $l_{B}$ fica muito menor que a largura do nosso poço $(W=3000 \AA)$ e o sistema representa essencialmente um sistema quase-tridimensional de elétrons. A Fig.(1.19) mostra um esquema da comparação de $l_{B}$ com a largura do poço $W$. Além disso, sabe-se, tal como explicado na parte inicial do cápitulo, que o espectro de energia do sistema, tornase em um espectro de energia tridimensional (Eq. 1.69) na presença de campo magnético paralelo intenso.

A aplicação de uma tensão de porta gera um deslocamento da função de onda dos elétrons na região da barreira, e no caso de $V_{g}<0$, a função de onda é puxada para a parte de baixo da amostra, como é esquematizado na Fig.(1.19). Nessa situação, para $V_{g}$ suficientemente intensa, o transporte é através da região perto da borda do poço e não pelo centro dele (ver Fig.1.19). Segundo G. C. Gardner et. al [86], a mobilidade $\mu$ é fortemente influenciada pela concentração de alumínio $(x)$ em um poço quântico, sendo máxima no GaAs puro $(x=0)$ e diminuindo com o aumento de $x$. Eles encontraram que para concentrações muito baixas existe uma relação linear entre o inverso da mobilidade e a concentração de Alumínio. Isto é mostrado pelos pontos azuis na Fig.(4.20). Assim, a dependência linear entre $\mu^{-1}$ e $x$ é dada pela equação seguinte:

$$
\mu^{-1}=(91,051 x+0,137) \times 10^{-6} V s / \mathrm{cm}^{2}
$$

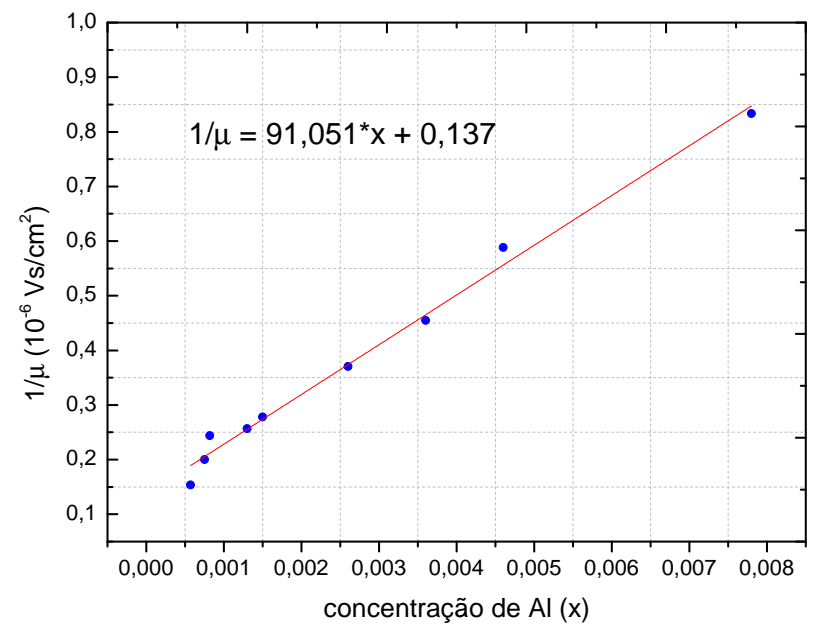

Figura 4.20: Dependência de $\mu^{-1}$ com a concentração de Alumínio (x) a partir dos dados de G. C. Gardner et. al. Mostra-se também o ajuste linear.

Portanto, em uma primeira aproximação, nós vamos usar essa dependência linear e extrapolar ela até os valores de concentração de Alumínio que contem o nosso poço quântico parabólico. Desse modo, os valores de $\mu$ obtidos usando a Eq.(4.9) são mostrados na Tabela (4.3) para alguns valores de $x$. 


\begin{tabular}{c|c}
\hline \hline$x$ & $\mu\left(10^{3} \mathrm{~cm}^{2} / \mathrm{Vs}\right)$ \\
\hline \hline 0,05 & 213 \\
0,10 & 108 \\
0,15 & 72 \\
0,20 & 54 \\
0,25 & 43 \\
0,30 & 36 \\
\hline \hline
\end{tabular}

Tabela 4.3: Mostra-se os valores de mobilidade $\mu$ obtidos a partir da Eq.(4.9) para valores de concentração de Aluminio variando desde 0,05 (centro do poço) até 0,30 (valor aproximado de $x$ na barreira do nosso poço parabólico).

A partir das nossas medidas experimentais da magnetoresistência a campo magnético zero (curva preta na Fig.4.10), é possível calcular a mobilidade (usando Eq.1.43) para cada valor de tensão de porta. Dessa maneira, a Tabela (4.4) mostra os valores da mobilidade $\mu$ para alguns dos valores de $V_{g}$. Para o cálculo de $\mu$ nesse intervalo de tensão de porta é assumido que a concentração de elétrons é mantida constante é igual a $n_{s}=1,9 \times 10^{11}$ $\mathrm{cm}^{-2}$.

\begin{tabular}{c|c}
\hline \hline$V_{g}(\mathrm{~V})$ & $\mu\left(10^{3} \mathrm{~cm}^{2} / \mathrm{Vs}\right)[$ Exp. $]$ \\
\hline \hline 0,00 & 220 \\
$-0,40$ & 161 \\
$-0,50$ & 65 \\
$-0,55$ & 34 \\
$-0,60$ & 15 \\
$-0,62$ & 11 \\
$-0,65$ & 6,5 \\
$-0,67$ & 4,5 \\
$-0,70$ & 2,3 \\
$-0,75$ & 0,9 \\
\hline \hline
\end{tabular}

Tabela 4.4: Mobilidades experimentais a campo magnético zero para diferentes valores de tensão de porta. Elas são cálculadas a partir dos valores de resistência da Fig.(4.10) e assumindo que $n_{s}$ é constante.

Comparando os resultados das Tabelas (4.3) e (4.4) observa-se que quando a $V_{g}=0,0$ $\mathrm{V}$, a mobilidade é comparável à mobilidade correspondente a uma concentração $x=0,05$ aproximadamente, o que indica que o transporte nessa situação é através da região central do poço parabólico, onde a concentração de Alumínio é muito baixa. Similarmente, notese que para $V_{g}=-0,55 \mathrm{~V}$, a mobilidade já é comparável à mobilidade correspondente a $x=0,3$ (que é aproximadamente a concentração de Alumínio nas bordas do poço), o que sugere que o transporte nesse caso é através da borda do poço parabólico. Portanto, 
podemos afirmar que no intervalo de variação da tensão de porta desde $V_{g}=0,0 \mathrm{~V}$ até $V_{g}=-0,55 \mathrm{~V}$, só temos deslocamento da função de onda eletrônica para a parte de baixo da amostra (segundo o esquema da Fig.1.19). Assim, a diminuição da mobilidade pode-se associar ao incremento da concentração de Alumínio desde o centro do poço $(x=0,0)$ até a borda $(x \approx 0,3)$. A tensão de porta $V_{g}=-0,55 \mathrm{~V}$ representa um valor de tensão limiar, pois a partir desse valor, não é possível deslocar mais os elétrons. Então, acredita-se que a partir desse valor de tensão limiar, a diminuição da mobilidade é devido à depleção de carga na borda do poço. Isto significa que a partir de $V_{g}=-0,55 \mathrm{~V}$, a concentração eletrônica $n_{s}$ não é mais constante, e consequentemente diminui o seu valor.

Segundo Ando et. al [26] a densidade de carga eletrônica depletada $N_{\text {depl }}$ é dada pela equação:

$$
N_{\text {depl }}=2,155 \times 10^{11}\left(\frac{100 \mathrm{~nm}}{d_{i s l}}\right)\left(\frac{\epsilon_{i s l}}{3,9}\right)\left(V_{g}-V_{L}\right) ; \quad\left[\mathrm{cm}^{-2}\right]
$$

onde $d_{i s l}$ é a distância desde a superficie da amostra até o poço, que para nossa amostra é $d_{i s l}=800 \AA, \epsilon_{i s l}=12,6, V_{g}$ é a tensão de porta e $V_{L}$ é a tensão limiar. Assim, a Eq.(4.10) fica:

$$
N_{\text {depl }}=8,7 \times 10^{11}\left(V_{g}+0,55\right) ; \quad\left[\mathrm{cm}^{-2}\right]
$$

Usando essa última expressão, é possível calcular a quantidade de carga que será depletada para cada valor de $V_{g}$ tal que $\left|V_{g}\right|>0,55$, e com isso, se consegue obter as densidades de carga $n_{s}$ no poço. A Tabela (4.5) mostra a densidade de carga constante na região $\left|V_{g}\right|<0,55$, mas para a região onde $\left|V_{g}\right|>0,55$ a densidade começa a diminuir devido à depleção. Também são mostrados os valores de mobilidade $\mu$ cálculados com os valores de $n_{s}$.

\begin{tabular}{c|c|c}
\hline \hline$V_{g}(\mathrm{~V})$ & $n_{s}\left(10^{11} \mathrm{~cm}^{-2}\right)$ & $\mu\left(10^{3} \mathrm{~cm}^{2} / \mathrm{Vs}\right)$ \\
\hline \hline 0,00 & 1,9 & 220 \\
$-0,40$ & 1,9 & 161 \\
$-0,50$ & 1,9 & 65 \\
$-0,55$ & 1,9 & 34 \\
$-0,60$ & 1,46 & 19 \\
$-0,62$ & 1,29 & 16,4 \\
$-0,65$ & 1,03 & 12 \\
$-0,67$ & 0,86 & 9,9 \\
$-0,70$ & 0,59 & 6,6 \\
\hline \hline
\end{tabular}

Tabela 4.5: Mostra-se as mobilidades $\mu$ e as densidades de carga $n_{s}$ para alguns valores de tensão de porta. Na região $\left|V_{g}\right|<0,55, n_{s}$ é constante; mas na região $\left|V_{g}\right|>0,55, n_{s}$ diminui. 
No intervalo da tensão de porta onde temos depleção de carga $\left(\left|V_{g}\right|>0,55\right)$, portanto, entende-se que a diminuição da mobilidade está associada à diminuição da densidade de carga eletrônica. Segundo A. Gold [87] a mobilidade $\mu$ tem uma dependência com a densidade de carga dada por: $\mu \propto n_{s}^{3 / 2}$. Assim, na Fig. (4.21) mostra-se o gráfico dos valores de $\mu$ e $n_{s}$ para $\left|V_{g}\right|>0,55$ (da Tabela 4.5), e é encontrado que a dependencia é $\mu \propto n_{s}^{1,29}$.

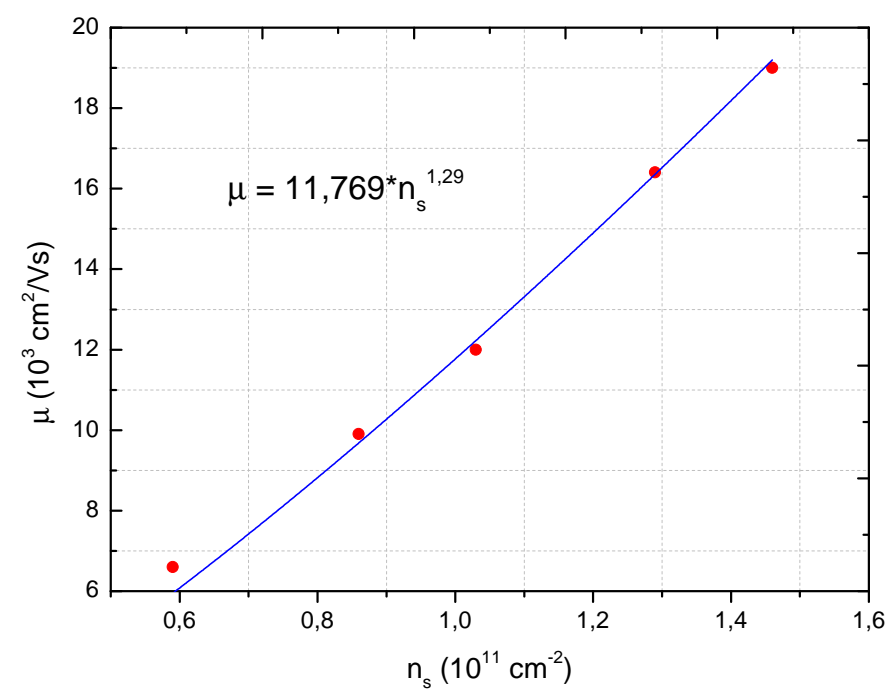

Figura 4.21: Mostra-se o gráfico da dependência da mobilidade $\mu$ com a densidade de carga $n_{s}$ para $\left|V_{g}\right|>0,55$.

Em resumo, todas estas aproximações nós permitem afirmar que quando $V_{g}=0,0 \mathrm{~V}$, o transporte eletrônico é através da região central do poço parabólico, e a mobilidade é máxima. A medida que a tensão de porta começa a aumentar (negativamente), os elétrons são puxados para a borda do poço, fato que vem acompanhado com uma diminuição na mobilidade devido ao aumento da concentração de Alumínio $(x)$ dentro do poço. Quando $V_{g}=-0,55 \mathrm{~V}$, o transporte é através da borda do poço parabólico, e a diminuição da mobilidade para valores de $\left|V_{g}\right|>0,55$ é associada a uma diminuição da densidade de $\operatorname{carga} n_{s}$.

Uma outra tentativa para a explicação das nossas medidas pode ser proposta levando em conta o spin eletrônico. Como foi visto no inicio do capítulo, o fato da concentração de alumínio ter uma variação parabólica dentro do poço, e o fator $g$ de Landé ter uma variação aproximadamente linear com essa concentração (dentro do intervalo de interesse), nós permite obter uma variação parabólica do fator $g$ de Landé ao longo do poço, dada pela Eq.(1.73). Incluindo essa variação do fator $g$ na hamiltoniana do sistema, obtemos uma renormalização da frequência natural do poço quântico parabólico (ver Eq.1.75). 
Nessa frequência renormalizada $\Omega_{\sigma}$, dada pela Eq.(1.76), aparece uma dependência com o spin eletrônico, o qual resulta muito interessante devido a que dependendo da orientação de spin e da modulação do campo magnético, o potencial de confinamento parabólico é modificado, e consequentemente a quantização do gás de elétrons também. Portanto, para o caso particular de alinhamento de spin antiparalelo ( $\sigma$ negativo) o efeito é atenuar o potencial parabólico até um valor de campo magnético crítico (Eq.1.77) no qual a frequência $\Omega_{\sigma}$ torna-se zero, e assim, sem potencial de confinamento não temos mais um sistema bidimensional de elétrons devido à ausencia de quantização no eixo $z$. Assim, o elétron com polarização de spin antiparalelo apresenta um carater tridimensional. Desse modo, espera-se que esse carater tridimensional do elétron influencie nas propriedades de transporte do nosso sistema.

Com base nessa última teoria, se cálculou o valor de campo magnético crítico para nossa amostra de largura $W=3000 \AA$. Para esse poço, obteve-se que $\hbar \omega_{0}=4,9 \mathrm{meV}$, o que resultou em um valor de campo magnético crítico $B_{c} \approx 2000 \mathrm{~T}$. Também se cálculou o valor de $B_{c}$ para amostras de menor largura. Para um poço de largura $W=1000 \AA$ com $\hbar \omega_{0}=12,15 \mathrm{meV}$ obteve-se $B_{c} \approx 1370 \mathrm{~T}$. Para um poço de largura $W=500 \AA \mathrm{com}$ $\hbar \omega_{0}=22 \mathrm{meV}$, cálculou-se $B_{c} \approx 1180 \mathrm{~T}$. Assim, os valores obtidos para $B_{c}$ estão muito longe dos campos magnéticos atingidos na realidade, motivo pelo qual esse modelo não pode ser aplicado no nosso sistema.

A partir do ponto de vista do transporte orbital, na presença de campo magnético paralelo, o transporte é dado através do último nivel de Landau. Nessa situação, o sistema tem um comportamento quase-tridimensional e a resistência cresce, concordando com as nossas medidas mostradas na Fig.(4.11).

O estudo da magnetoresistência a campo magnético zero permitiu descobrir que para uma tensão de porta $V_{g}=-0,55 \mathrm{~V}$ é gerada uma barreira de potencial, ainda sem ter depleção de cargas na região. O incremento da magnetoresistência com o campo magnético para $-0,55 \mathrm{~V}<V_{g}<0 \mathrm{~V}$ pode estar associado a um efeito válvula de spin através de mecanismos de espalhamento de spin na região da barreira. 


\section{Capítulo 5}

\section{Conclusões}

Neste trabalho, relatamos estudos sobre a magnetoresistência em amostras de poços quânticos parabólicos (PQWs) crescidas pela técnica de MBE em substratos de GaAs. Em uma primeira parte estudamos duas amostras com a mesma largura $W=1000 \AA$, mas com diferentes densidades de carga $n_{s}$. Medidas de magnetoresistência e de resistência de Hall em campo magnético perpendicular, permitiram determinar experimentalmente as densidades de carga $n_{s}$ e as mobilidades $\mu$ dessas amostras. A análises das oscilações Shubnikov-de Haas através da transformada de Fourier permitiu conhecer o número de sub-bandas ocupadas nos poços, assim como as densidades de carga em cada uma delas. Estudamos os PQWs através do formalismo dos cálculos autoconsistentes, assim, determinou-se a distribuição da densidade de cargas $n(z)$, o perfil de potencial total $V(z)$, as energias de confinamento $E_{i}$ e as concentrações de carga em cada sub-banda $n_{s}^{(i)}$. Verificamos uma boa concordância entre os valores obtidos por cálculos autoconsistentes e os medidos experimentalmente.

Na segunda parte, estudou-se uma amostra contendo na sua superficie uma porta metálica de ouro com uma barreira muito estreita. Estudamos os efeito na magnetoresistência devido à aplicação de uma tensão de porta, ou seja, através da aplicação de potenciais externos em uma região muito estreita (região da barreira). Na presença de campo magnético perpendicular, os platôs na magnetoresistência podem ser explicados de maneira satisfatória através do modelo de estados de borda. Na configuração de campo magnético paralelo observa-se que as curvas de magnetoresistência medidas para cada valor de $B$, crescem com um comportamento exponencial, assim determinou-se uma relação para $R_{x x}$ em função de $B$ e $V_{g}$, mas ainda não temos uma formulação teórica que nos permita comparar este resultado.

As aproximações feitas nós permitem afirmar que quando $V_{g}=0,0 \mathrm{~V}$, o transporte eletrônico é através da região central do poço parabólico, e a mobilidade é máxima. A medida que a tensão de porta começa a aumentar (negativamente), a função de onda 
eletrônica é deslocada para a parte de baixo da amostra, o que equivale a dizer que os elétrons são puxados para a borda do poço. Esse fato, vem acompanhado com uma diminuição da mobilidade devido ao aumento da concentração $x$ de Alumínio dentro do poço. Quando $V_{g}=-0,55 \mathrm{~V}$, o transporte é através da borda do poço parabólico, e a diminuição da mobilidade para valores de $\left|V_{g}\right|>0,55$ é atrubuida à diminuição da densidade de carga $n_{s}$, ou seja, a partir desse valor temos que acontece uma depleção de cargas.

O fato do fator $g$ de Landé ter uma variação parabólica ao longo de um PQW, permite renormalizar a frequência natural do poço $\omega_{0}$ em $\Omega_{\sigma}$, a qual introduz o spin eletrônico. Para um elétron com orientação de spin antiparalela, o modelo prevê a existencia de um campo magnético crítico $B_{c}$ para o qual o elétron perde o seu carater bidimensional e torna-se tridimensional. No entanto, com os parâmetros da amostra estudada o valor de $B_{c}$ resulta muito longe do valor dos campos atingidos no laboratorio.

Foi possível a idealização de um transistor válvula de spin. A aplicação de uma tensão de porta gera o deslocamento da função de onda, e com isso, o fluxo de elétrons atinge a região da barreira do poço, mudando o sinal do fator $g$ do sistema. O aumento da magnetoresistência com o campo magnético pode estar associado a um efeito válvula de spin na amostra, devido a uma diminuição do passo de portadores de carga através da barreira de potencial estreita. 


\section{Referências Bibliográficas}

[1] K. von Klitzing, G. Dorda, and M. Peppers. Phys. Rev. Lett., 45:494, (1980). xix, 1, 20

[2] D. C. Tsui, H. L. Stormer, and A. C. Gossard. Phys. Rev. Lett., 48:1559, (1982). xix, 1

[3] L. Esaki and R. Tsu. IBM J. Res. Develop., 14:61, (1970). xix

[4] A. E. Blakeslee and C. F. Aliotta. IBM J. Res. Develop., 14:686, (1970). xix

[5] A. Y. Cho. Appl. Phys. Lett., 19:467, (1971). xix

[6] W. P. Dumke, J. M. Woodall, and V. L. Rideout. Solid State Electron, 15:1339, (1972). xix

[7] Miller R. C, D. A. Kleinman, and A. C. Gossard. Phys. Rev. B, 29:7085, (1984). xx

[8] M. Sundaram, A. C. Gossard, J. H. English, and R. M. Westervelt. Superlattice and Microstructures, 4:683, (1988). xx, 43

[9] M. Shayegan, T. Sajoto, M. Santos, and C. Silvestre. Appl. Phys. Lett., 53:791, (1998). xx

[10] D. Kahng and M. Atalla. IRE Solid State Devices Res. Conf. Carnegie Institute of Technology, Pittsburgh, PA, (1960). 1

[11] A. B. Fowler, F. F. Fang, W. E. Howard, and P. J. Stiles. Phys. Rev. Lett., 16:901, (1966). 1

[12] David K. Ferry. Transport in Nanostructures. Cambridge University Press, (1987). 6

[13] L. Smrcka and T. Jungwirth. J. Phys. Condens. Matter., 6:55, (1994). 7, 8

[14] G. M. Gusev, A. A. Quivy, T. E. Lamas, J. R. Leite, A. K. Bakarov, A. I. Toropov, O. Estibals, and J. C. Portal. Phys. Rev. B, 65:205316, (2002). 7, 35, 36, 37

[15] L. Smrcka, P. Vasek, J. Kolacek, T. Jungwirth, and M. Curk. Surface Science, 361:509, (1996). 8

[16] U. Merkt. Phys. Rev. B, 32:6699, (1985). 8, 9

[17] J. M. Heisz and E. Zaremba. Semicond. Sci. Technol., 8:575, (1993). 10 
[18] Chihiro Hamaguchi. Basic Semiconductor Physics. Springer, second edition, (2010). 11

[19] Mark Lundstrom. Fundamental and Carrier Transport. Cambrige University Press, (2000). 11

[20] Yuri M. Galperins. Introduction to Modern Solid Physics. (2009). 13, 16

[21] Giuseppe Grosso and Giuseppe Pastori Parravicini. Solid State Physics. Academic Press, (2003). 17

[22] James D. Patterson and Bernard C. Bailey. Solid State. Springer, (2007). 19

[23] H. Bachmair, E. Göbel, G. Hein, J. Melcher, B. Schumacher, J. Schurr, L. Schweitzer, and P. Warnecke. Physica E, 20:14, (2003). 20

[24] B. N. Taylor. IEEE Trans. Instrum. Meas., 38:164, (1989). 20

[25] R. E. Prange. Phys. Rev. B, 23:4802, (1981). 20

[26] T. Ando, A. Fowler, and F. Stern. Rev. Mod. Phys, 54:437, (1982). 20, 94

[27] T. Chakraborty and Pietiläinen. The Quantum Hall Effects: Integral and Fractional. Springer-Verlag, second edition, (1995). 20

[28] M. Janßen, O. Viehweger, U. Fastenrath, and J. Hajdu. Introduction to the Theory of the Integer Quantum Hall Effect. VCH, Weinheim Heidelberg, (1994). 20

[29] S. Das Sarma and A. Pinczuk. Perspectives on Quantum Hall Effects. Wiley, New York, (1996). 20

[30] B. Huckestein. Rev. Mod. Phys., 67:357, (1995). 20

[31] R. R. Gerhardts. Z. Phys. B, 21:275, (1975). 21

[32] L. Shubnikov and W. J. de Haas. Leiden Commun., 3:207, (1930). 24

[33] E. Zaremba. Phys. Rev. B, 45:14143, (1986). 24

[34] G.-Q. Hai, N. Studart, and F. M. Peeters. Phys. Rev. B, 52:8363, (1995). 24

[35] T. Ando and Y. Uemura. J. Phys. Soc. Jpn., 36:959, (1974). 24

[36] B. K. Ridley. Quantum processes in semiconductors. Clarendon Press, Oxford, third edition, (1993). 24

[37] M. Ahoujja, S. Elhamri, R. S. Newrock, D. B. Mast, W. C. Mitchel, I. Lo, and A. Fathimulla. J. Appl. Phys, 81:1609, (1997). 26

[38] R. J. Haug. Semicond. Sci. Technol., 8:131, (1993). 27, 82

[39] M. Büttiker. Semiconductors and semimetals, 35:191, (1992). 27

[40] M. Büttiker. Phys. Rev. B, 38:9375, (1988). 27 
[41] J. Davies. The physics of low dimensional semiconductors. Cambridge University Press, Cambridge, (1998). 27

[42] M. Büttiker, Y. Imry, R. Landauer, and S. Pinhas. Phys. Rev. B, 31:6207, (1985). 29

[43] I. A. Yukova, A. Greilich, D. R. Yakovlev, A. A. Kiselev, and M. Bayer. Phys. Rev. $B, 75: 245302,(2007) .32$

[44] H. Malissa, W. Jantsc, M. Muhlberger, F. Schaffler, Z. Wilamowski, M. Draxler, and P. Bauer. Appl. Phys. Lett., 85:1739, (2004). 32

[45] P. Pfeffer and W. Zawadzki. Phys. Rev. B, 72:035325, (2005). 32, 33

[46] J. M. Luttinger and W. Kohn. Phys. Rev., 97:869, (1955). 32

[47] P. Pfeffer and W. Zawadzki. Phys. Rev. B, 41:1561, (1990). 32

[48] C. Bosio, J. L. Staehli, M. Guzzi, G. Burri, and R. A. Logan. Phys. Rev. B, 38:3263, (1988). 33

[49] O. Berolo and J. C. Woolley. Proceedings of the 11th International Conference on Physics and Semiconductors. Polish Scientific Publishers, Warsaw, (1972). 33

[50] D. J. Chadi, A. H. Clark, and R. D. Burnham. Phys. Rev. B, 13:4466, (1976). 34

[51] C. Hermann and C. Weisbuch. Phys. Rev. B, 15:816, (1977). 34

[52] R. Merlin. Solid State Commun., 64:99, (1987). 38

[53] I. C. da Cunha Lima, G. M. Gusev, and J. R. Leite. Journal of Superconductivity, 18:169, (2005). 38, 39

[54] C. A. Duarte, G. M. Gusev, A. A. Quivy, and T. E. Lamas. Brazilian Journal of Physics, 36:488, (2005). 39

[55] A. J. Rimberg and R. M. Westervelt. Phys. Rev. B, 40:3970, (1989). 43, 46, 47, 48

[56] B. I. Halperin. Jpn. J. Appl. Phys., 26:1913, (1987). 43

[57] A. Gossard, M. Sundaram, and P. F. Hopkins. Semiconductors and Semimetals, 40:153, (1994). 43

[58] H. L. Störmer. Surf. Sci., 132:519, (1983). 43

[59] E. G. Gwinn, P. F. Hopkins, A. J. Rimberg, R. M. Westervelt, M. Sundaram, and A. C. Gossard. Phys. Rev. B, 41:10700, (1990). 44, 45, 48

[60] R. Dingle, H. L. Störmer, A. C. Gossard, and W. Wiegmann. Appl. Phys. Lett, 33:665, (1978). 44

[61] E. G. Gwinn, R. M. Westervelt, P. F. Hopkins, A. J. Rimberg, M. Sundaram, and A. C. Gossard. Phys. Rev. B, 39:6260, (1989). 46

[62] P. Hohenberg and W. Kohn. Phys. Rev., 136:B864, (1964). 48 
[63] W. Kohn and L. J. Sham. Phys. Rev., 140:A1133, (1965). 48

[64] L. J. Sham and W. Kohn. Phys. Rev., 145:561, (1966). 48

[65] L. Heiden and B. I. Lundqvist. Phys. C: Solid State Phys., 4:2064, (1971). 48

[66] M. P. Stopa and S. Das Sarma. Phys. Rev. B, 47:2122, (1993). 48

[67] N. Chand, T. Henderson, J. Klem, W. Ted Masselink, and R. Fisher andY. C. Chang andH. Morkoc. Phys. Rev. B, 30:4481, (1984). 48

[68] Celso de Araujo Duarte. Propriedades magnéticas e de spin em semicondutores do grupo III-IV. Tese de Doutorado apresentada ao Instituto de Física da Universidade de São Paulo, (2004). 49

[69] F. Stern. J. Comp. Phys., 6:56, (1970). 49

[70] Cássio Sanguini Sergio. Transporte quântico em poços parabólicos largos. Tese de Doutorado apresentada ao Instituto de Física da Universidade de São Paulo, (2003). 50

[71] Angela María Ortiz de Zevallos Márquez. Estudo de poços parabólicos largos de AlGaAs em campos magnéticos altos. Tese de Doutorado apresentada ao Instituto de Física da Universidade de São Paulo, (2007). 52, 53

[72] E. H. C. Parker. The technology and physics of molecular beam epitaxy. Plenum Press, New York, (1985). 59

[73] Sandro Martini. Estudo da segregação de índio em camadas epitaxiais de InGaAs crescidas sobre substratos de GaAs(001). Tese de Doutorado apresentada ao Instituto de Física da Universidade de São Paulo, (2002). 60

[74] W Geißelbrecht, U Pfeiffer, A Thränhardt, U Klütz, A.C Gossard, and G.H Döhler. J. Crystal Growth, 201-202:162, (1999). 62

[75] S.M. Wang, T.G. Andersson, W.Q. Chen, U. Södervall, and J. Thordson. J. Crystal Growth, 135:455, (1994). 62

[76] Tomas Erickson Lamas. Epitaxia por feixe molecular de camadas dopadas do tipo p para construção de dispositivos optoelectrônicos. Tese de Doutorado apresentada ao Instituto de Física da Universidade de São Paulo, (2004). 63

[77] Partha Ray and P. K. Basu. Phys. Rev. B, 45:9169, (1992). 63

[78] S. B. Ogale and A. Madhukar. J. Appl. Phys., 56:368, (1984). 63

[79] A. S. Sedra and K. C. Smith. Microelectronic circuits. Oxford University Press, (1997). 66

[80] Luis Enrique Gómez Armas. Magnetotransporte em poços quânticos duplos e triplos com diferentes valores do fator $g$ de Landé. Tese de Doutorado apresentada ao Instituto de Física da Universidade de São Paulo, (2009). 76 
[81] Ivan Ramos Pagnossin. Propriedades de transporte elétrico de gases bidimensionais de elétrons nas proximidades de pontos quânticos de InAs. Dissertação de mestrado apresentada ao Instituto de Física da Universidade de São Paulo, (2004). 76

[82] S. W. Smith. The scientist and engineers's guide to digital signal processing. California Technical Publishing, (1999). 77

[83] A. Cavalheiro. O efeito da fotocondutividade e a estrutura eletrônica de poços quânticos de GaAs/InGaAs/GaAs com dopagem planar do tipo n na barreira. Tese de Doutorado apresentada ao Instituto de Física da Universidade de São Paulo, (2001). 77

[84] R. W. Shaw and D. E. Hill. Phys. Rev. B, 1:658, (1970). 77

[85] R. J. Haug, A. H. MacDonald, P.Streda, and K. von Klitzing. Phys. Rev. Lett., 61:2797, (1988). 81

[86] G. C. Gardner, J. D. Watson, S. Mondal, N. Deng, G. A. Csáthy, and M. J. Manfra. Appl. Phys. Lett., 102:252103, (2013). 92

[87] A. Gold. Appl. Phys. Lett., 54:2100, (1989). 95 